\title{
Electron impact collision strengths in Si IX, Si X and Si XI
}

\author{
Guiyun Liang ${ }^{a}$, Gang Zhao ${ }^{a}$ a and Jiaolong Zeng ${ }^{b}$ \\ ${ }^{a}$ National Astronomical Observatories, Chinese Academy of Sciences, Beijing 100012, P. R. China and \\ ${ }^{b}$ Department of Applied Physics, National University of Defense Technology, Changsha 410073, P. R. China
}

\begin{abstract}
Electron impact collision strengths among 560 levels of Si IX, 320 levels of Si X and 350 levels of Si XI have been calculated using the Flexible Atomic Code (FAC) of Gu (2003). Collision strengths $\Omega$ at ten scattered electron energies covering an entire energy range, namely 10, 50, 100, 200, 400, 600, 800, 1000, 1500 and $2000 \mathrm{eV}$, are reported. Assuming a Maxwellian energy distribution, effective collision strengths $\Upsilon$ are obtained at a finer electron temperature grids of 0.5, 1.0, 2.0, 3.0, 4.0, 5.0 and 6.0 MK, which covers the typical temperature range of astrophysical hot plasma. Additionally, radiative rates $A$ and weighted oscillator strengths $g f$ are given for more possible transitions among these levels. Comparisons of our results with available predictions reported in earlier literatures are made, and the accuracy of the data is assessed. Most transitions exhibit a better agreement, whereas large differences in $g f$ appear for a few cases, which are due to the different inclusion of configuration interaction in different theoretical calculations. In excitations among levels of ground and lower excited configurations, large discrepancies of $\Upsilon$ maybe resulted from the consideration of resonance effects in earlier works.
\end{abstract}

\section{INTRODUCTION}

A wealth of high resolution spectra in UV, EUV and X-ray regions has been obtained for solar, stellar and other astrophysical sources by many space missions, such as SOHO, Chandra and XMM-Newton. Many of the observed emission lines are due to highly charged silicon ions (Si VII-Si XIV), as reported in literatures for Procyon and $\alpha$ Centauri [1, 2]. For line identifications and spectral analyses, a complete list of lines including emission or absorption lines of highly charged silicon, is very necessary. This is available in the Chianti ${ }^{1}$ and ATOMDB ${ }^{2}$ data sets. However, the available data of atomic parameters is very limited for elements such as silicon, sulfur, argon and calcium. Moreover, the accuracy of the available data for these L-shell ions attracts attention because of the poor modelling for the astrophysical spectra [3]. Recently, Aggarwal et al. [4], Liang et al. [5] and Landi \& Bhatia [6] performed the atomic data calculations of Ar L-shell ions with larger configuration interaction (CI). They listed energy levels, radiative rates, and/or collision strengths. Generally, more accurate results could be obtained by considering larger CI, as stated by Aggarwal et al. [4].

Although many calculations for the highly charged silicon ions have been performed in past decades, and the data is used extensively in present astrophysical modelling codes, such as Chianti and APEC. Yet the data is limited to lowlying energy levels. For electron impact collision strengths, almost all available data is confined to levels with $n=2$ configurations. Moreover, available theoretical data of radiative rates is also confined to allowed and inter-combination (E1) transitions alone. In addition, the data used by the Chianti and APEC codes is from different literatures. Here, we attempt to present a self-consist, accurate and extensive data for highly charged Si ions. Additionally, to provide experimental support, many lines of Si VIII - XIII in the 40-80 soft X-ray range have been measured in an experiment of silicon target irradiated by intense femtosecond laser at the Institute of Physics, Chinese Academy of Sciences [7].

\section{ENERGY LEVELS}

This study adopts the flexible atomic code (FAC) developed by Gu $[8,9]$ to perform calculations of the structure and the $e$-ion interaction process. This code is a standard atomic structure code alike GRASP code of Dyall et al. [10], and available at the website http://kipac-tree.stanford.edu/fac/. A fully relativistic approach based on Dirac

\footnotetext{
*Electronic address: gzhao@bao.ac.cn

${ }^{1}$ http://wwwsolar.nrl.navy.mil/chianti.html

2 http://cxc.harvard.edu/atomdb/
} 
equation is used throughout the entire package. The specific configurations included, results obtained and accuracy achieved, are discussed below for each ion.

\subsection{Si IX}

In the work of Aggarwal 11], 46 low-lying energy levels and radiative rates among these levels for carbon-like ions including Si IX, were reported. An extensive CI was considered in this work, and better results were obtained when compared with earlier calculations. Yet only 46 levels belong to configurations of $\left(1 s^{2}\right) 2 s^{2} 2 p^{2}, 2 s 2 p^{3}, 2 p^{4}$, and $2 s^{2} 2 p 3 l$, were reported. In comparison with experimental values (NIST ${ }^{1}$ ), level energies agree within $2 \%$ except for two levels of $2 s^{2} 2 p^{2}{ }^{1} D_{2}$ and ${ }^{1} S_{0}$. The Chianti code adopts an earlier calculation of Bhatia \& Doschek [12], which also reported 46 levels, but less CI was considered. Besides atomic data such as the energy levels, radiative decay rates and collision strengths, this work also presented line intensities for some strong transitions by solving rate equations of level populations. Orloski et al. 13] (hereafter OTC99) performed the calculation of energy levels by including additional configurations, namely $2 s 2 p^{2} 3 l$. Further, the calculated energy levels were adjusted again by observed wavelengths using an interactive optimization procedure packaged in the program ELCALC 14].

In our study, energies of 560 levels belonging to 31 configurations of Si IX [namely, $2 s^{2} 2 p^{2}, 2 s 2 p^{3}, 2 p^{4}, 2 s^{2} 2 p 3 l$, $\left.2 s 2 p^{2} 3 l, 2 p^{3} 3 l(l=s, p, d), 2 s^{2} 2 p 4 l^{\prime}, 2 s 2 p^{2} 4 l^{\prime}, 2 s^{2} 2 p 5 l^{\prime}, 2 s^{2} 2 p 6 l^{\prime}\left(l^{\prime}=0,1, \ldots, n-1\right)\right]$ are reported as listed in Table 1. In order to assess the accuracy, we also list the NIST data which is recognized the most reliable data so far, and other theoretical calculations. In comparison with available experimental values, present results are better than $2 \%$ for most levels. Though a less CI has been considered than the work of Aggarwal 11], present level energies show an excellent agreement with those reported by Aggarwal [1]. On the other hand, this indicates that the CI effect from other configurations is not distinct. The theoretical calculation of Bhatia \& Doschek 12] also agree with experimental values except for the level $2 s 2 p^{3}{ }^{5} S_{2}$. Its value is lower than the experimental one by $\sim 8 \%$. By considering large CI, the level energy increases to 1.3025 Ryd, and agrees with experimental one within $2 \%$. Results of Orloski et al. 13. had been adjusted by observed wavelengths, so their level energies are listed for comparison in Table 1. Fig.1-(a) visually illustrate such comparison. For those higher excited levels, the configuration mixing is very severe. So level designation and ordering are very difficult. And only a few levels can be obtained from NIST database, and some levels are labelled by question mark '?'. For these levels, we note that our results differ the experimental values by less than 0.07Ryd. This suggest that a good agreement has been obtained. Aggarwal et al. 11] considered a larger CI in their calculation. Unfortunately, the higher excited levels have not been reported.

\section{$2.2 \mathrm{Si} \mathrm{X}$}

For this ion, 50 experimental level energies belonging to the $2 s^{2} 2 p, 2 s 2 p^{2}, 2 p^{3}, 2 s 2 p 3 l(l=s, p, d), 2 p^{2} 3 d, 2 s^{2} 4 d$, $2 s^{2} 5 d$ and $2 s^{2} 6 d$ configurations can be obtained from the NIST website. In the Chianti database, additional 75 theoretical energy levels belonging to configurations, namely $2 s^{2} 2 p, 2 s 2 p^{2}, 2 p^{3}, 2 s^{2} 3 l, 2 s 2 p 3 l, 2 p^{2} 3 l(l=s, p, d)$, are compiled from earlier works. And different theoretical calculations from different atomic physicists have been included, such as Edlen [15], Zhang et al. 16, 17], and Sampson \& Zhang [18]. Cavalcanti et al. 19] also reported some level energies belonging to $2 s^{2} 2 p, 2 p^{3}, 2 s^{2} 3 l, 2 s 2 p 3 l, 2 p^{2} 3 l, 2 s^{2} 4 l, 2 s 2 p 4 p, 2 s 2 p 4 d, 2 s^{2} 5 d, 2 s 2 p 5 p$ and $2 s 2 p 5 d$ configurations using a multi-configuration Hartree-Fock relativistic approach, and adjusted their results by observed wavelengths.

By considered a larger CI, we calculate the level energies of Si X, and 320 levels belonging to configurations of $2 s^{2} 2 p$, $2 p^{3}, 2 s^{2} 3 l, 2 s 2 p 3 l, 2 p^{2} 3 l(l=s, p, d), 2 s^{2} 4 l^{\prime}, 2 s 2 p 4 l^{\prime}, 2 s^{2} 5 l^{\prime}, 2 s 2 p 5 l^{\prime}$ and $2 s^{2} 6 l^{\prime}\left(l^{\prime}=0,1, \ldots, n-1 ; n\right.$ is main quantum number) are reported as listed in Table 2 . For comparison, other available theoretical results and experimental ones are also presented.

An inspection of Table 2 indicates that present results show a good agreement with experimental values and other different predictions, which is visually displayed in Fig.1-(b). Some higher excited levels such as levels from the $2 s 2 p 4 l$ and $2 s 2 p 5 l$ configurations, were also reported by Cavalcanti et al. 19] (hereafter referring to CLT00), and these energy values were adjusted by observed wavelengths. A comparison with these results reveals that our results are better than $1 \%$ as shown by filled circle in Fig.1-(b). From Table 2, we found the differences are less than 0.08 Ryd.

\footnotetext{
${ }^{1}$ http://physics.nist.gov/cgi-bin/AtData/main_asd
} 


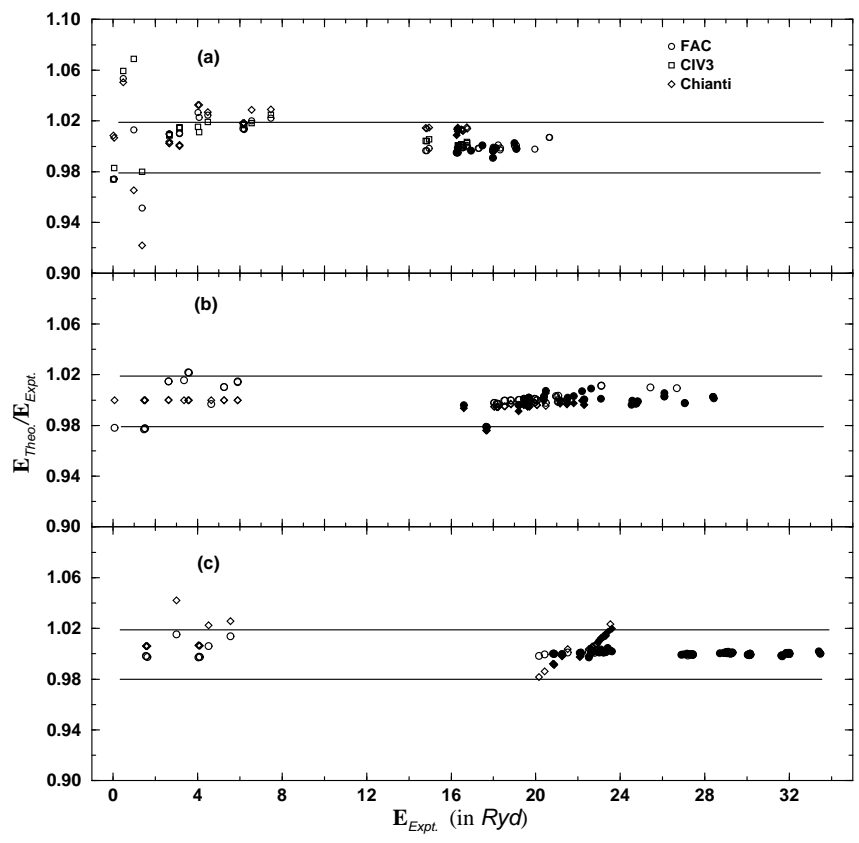

FIG. 1: Comparison of different calculations of energy levels versus the available experimental ones (from NIST database). The $\mathrm{x}$-axis denotes the experimental energy (in $R y d$ ), the y-axis denotes ratio of the theoretical calculations $v s$ experimental ones. (a) for Si IX; (b) for Si X; (c) for Si XI.

\subsection{Si XI}

For this ion, almost all available theoretical results of energy levels are for low-lying 46 levels with $n=3$ configurations, which are extensively used by current astrophysical modelling codes such as Chianti, MEKAL and APEC. Typical studies are the works of Sampson et al. 20] and Zhang \& Sampson [21]. Coutinho \& Trigueiros [22] also reported higher excited levels of $2 s 4 l, 2 p 4 l, 2 s 5 l$ and $2 p 5 l$ configurations, in which a multi-configuration Hartree-Fock relativistic approach was adopted. The calculated energies were adjusted again by observed wavelengths using the interactive optimization procedure included in the ELCALC [14] program. In the newest version of NIST database, about 48 experimental energy levels belonging to the $2 s 2 p, 2 p^{2}, 2 s 3 l, 2 p 3 l, 2 s 4 l, 2 p 4 p, 2 p 4 d, 2 s 5 d$ and $2 p 5 d$ configurations are listed. Here, we reported energies of 350 levels belonging to the 28 configurations of Si XI, namely $2 s^{2}, 2 s 2 p$, $2 p^{2}, 2 l 3 l^{\prime}, 2 l 4 l^{\prime}, 2 l 5 l^{\prime}, 2 l 6 l^{\prime}$ and $2 l 7 l^{\prime}\left(l=s, p ; l^{\prime}=0,1, \ldots, n-1\right)$.

In Table 3 we list the level energies along with available experimental and theoretical compilations for a comparison. Present predictions show a good agreement with experimental ones for a majority of levels. Even for the lowest-lying levels of ground and lower excited configurations, the largest difference does not exceed 2\%. Fig.1-(c) obviously indicates present results being better than the theoretical data included in the Chianti. For higher excited levels, we use the data of Coutinho \& Trigueiros 22] to assess the accuracy, because Coutinho \& Trigueiros 22] adjusted their results with observed wavelengths. A good agreement (within 0.3\%) appears as shown by the filled circled in Fig.3-(c) and in Table 3 .

\section{WEIGHTED OSCILLATOR STRENGTH}

We further reported the weighted absorption oscillator strength $\left(g f_{i j}\right)$ and radiative decay rate $\left(A_{i j}\right)$ for a given transition $i \rightarrow j$ using the FAC package. In the following, we discuss our results of weighted oscillator strengths for each of the silicon ions in detail. 

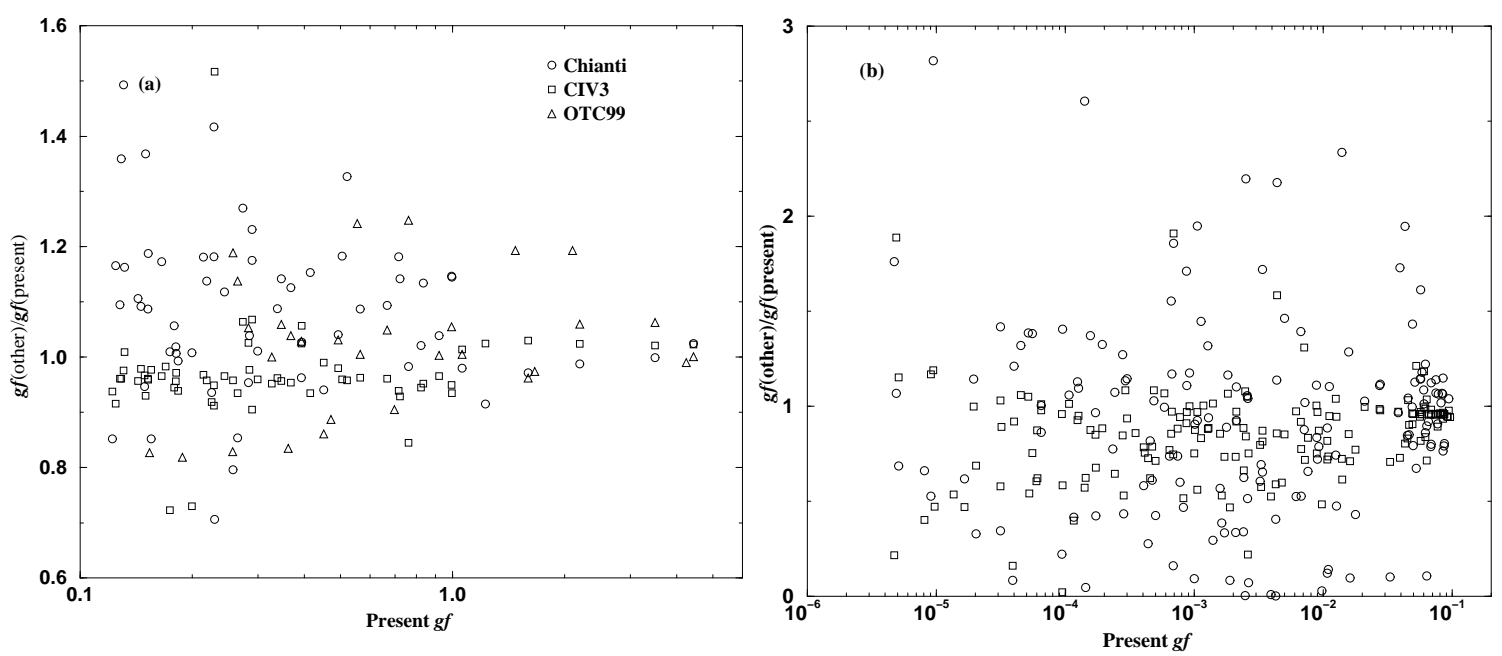

FIG. 2: Comparison of the Si IX weighted oscillator strengths from different calculations. The x-axis denotes the present results, the y-axis denotes ratio of other theoretical predictions versus present ones. (a) For strong transitions $g f>0.1$, (b) For weak transition $10^{-6}<g f<0.1$.

\subsection{Si IX}

Available theoretical data of radiative rates or weighted oscillator strengths was confined to transitions among 46 low-lying levels 11, 12 of Si IX. For strong or allowed transitions, the oscillator strengths are accurate to $10 \%$ as stated by Aggarwal [1] who adopted the CIV3 code [23]. However larger uncertainties still exist for those weak forbidden transitions $(g f<0.1)$. In the work of Orloski et al. 13], weighted oscillator strengths $(g f)$ to higher excited levels of $2 s 2 p^{2} 3 l$ configurations were also reported. In addition, a majority of literatures reported the radiative rates for electric dipole transitions among levels with $n=2$ complexes. To our best knowledge, no additional works reported the radiative rates or oscillator strengths for $\Delta n \geq 1$ transitions among levels of $n=2,3,4$ and 5 complexes. In order to successfully modelling the astrophysical high-quality spectra, here we extend the data of the radiative rates. The $g f$ and radiative rates for transitions among 560 levels were calculated as listed in Table 10. Not only the E1 type transitions are reported, but also other type transitions such as M1, E2 and M2, have been calculated, which directly results in the number of transitions increases by orders of magnitude.

In Table 4 we list some $g f$-values along with other available data of Aggarwal [11], Orloski et al. [13] and the data included in the Chianti database for a comparison. The Chianti code adopts results of Bhatia et al. [12] in the modelling of astrophysical spectra. A visual comparison is shown by Fig.2 for all available transitions. Because large CI has been considered by Aggarwal 11] and us, the two different calculations show a good agreement (within $20 \%$ ) for most strong transitions $(g f>0.1)$ as shown by square symbols in Fig.2-(a). Only three transitions such as $2 s^{2} 2 p^{2}{ }^{1} D_{2}-2 s^{2} 2 p 3 d d^{3} F_{2}$ (4-38, numbers corresponding to level indices), $2 s^{2} 2 p 3 p{ }^{1} P_{1}-2 s^{2} 2 p 3 d{ }^{1} D_{2}(25-40)$ and $2 s^{2} 2 p 3 p^{3} D_{1}-2 s^{2} 2 p 3 d^{3} F_{2}$ (26-38), show differences of $>20 \%$. Such differences are mainly due to the CI effect from another three configurations of $3 s 3 p, 3 p 3 d$ and $3 d^{2}$ considered by Aggarwal 11]. We gradually considered the three configurations, the $g f$ increases from 0.2293 to 0.3031 for $2 s^{2} 2 p^{2}{ }^{1} D_{2}-2 s^{2} 2 p 3 d^{3} F_{2}(4-38)$ transition, whereas it drops from 0.1995 and 0.1742 to 0.1454 and 0.1703 for $2 s^{2} 2 p 3 p{ }^{1} P_{1}-2 s^{2} 2 p 3 d{ }^{1} D_{2}(25-40)$ and $2 s^{2} 2 p 3 p{ }^{3} D_{1}-2 s^{2} 2 p 3 d^{3} F_{2}$ (26-38) transitions, respectively. This reveals that the CI is the most possible reason for large uncertainties. The comparison with the data used by the Chianti code is displayed by open-circle symbols in Fig.2-(a). Though a less CI was considered in the work of Bhatia et al. [12], the two different data still agree within $20 \%$ for most strong transitions. Yet, for more transitions besides above three ones, the two different calculations differ beyond $20 \%$. Additionally, the comparison with the results of Orloski et al. 13] is illustrated by up-triangle symbols in Fig.2-(a). For those weak transitions $(g f<0.1)$, more transitions show large discrepancies between different calculations as shown in Fig.2-(b). We also test the reason for the large differences, and found that the CI effect is the main reason. Generally, present results agree with the results of Aggarwal [1], whereas a slightly poor agreement with the data included in the Chianti appears. The presence of large uncertainties suggests a scope of improvement. 

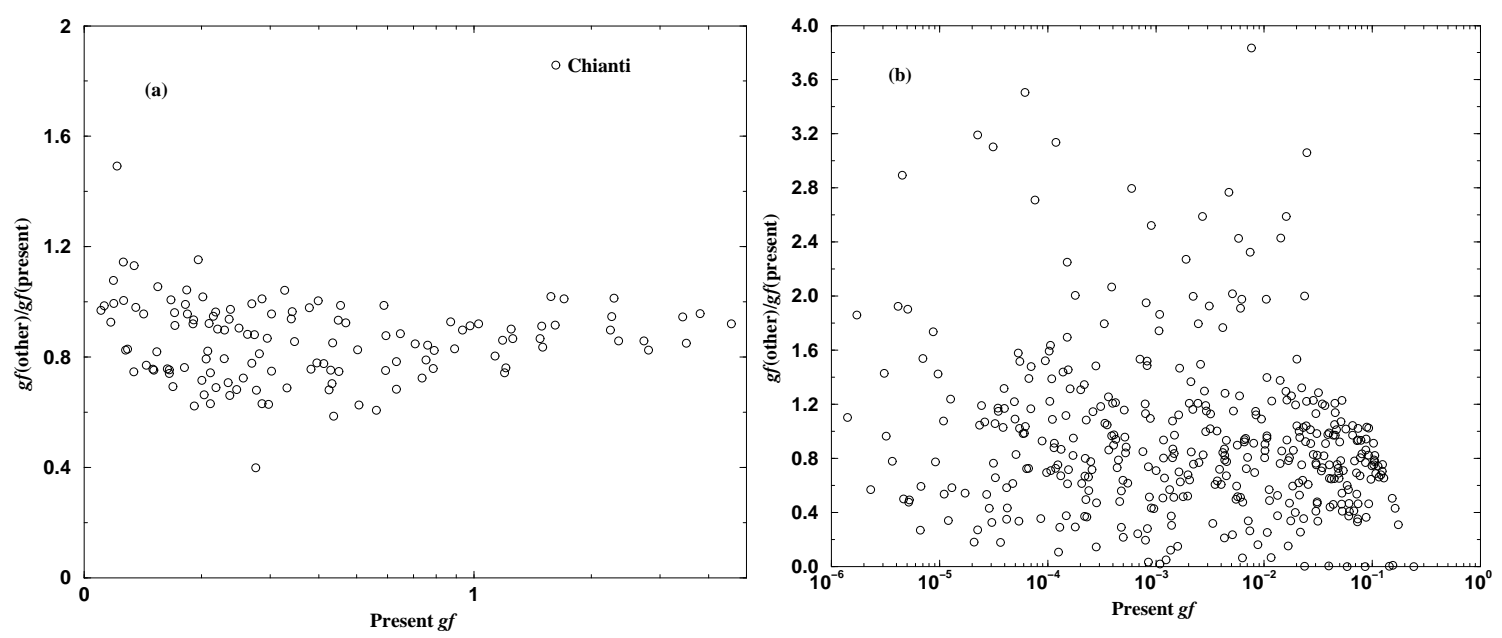

FIG. 3: Comparison of the Si X weighted oscillator strengths from different calculations. The x-axis denotes the present results, the y-axis denotes ratio of the data included by the Chianti versus present ones. (a) For strong transitions $g f>0.1$, (b) For weak transition $10^{-6}<g f<0.1$.

\subsection{Si X}

For B-like silicon, most available radiative rates or oscillator strengths of Si X are for transitions among the lowest 15 levels. The radiative rates from higher excited levels were firstly reported by Zhang \& Sampson 16., which were extensively used in current astrophysical modelling codes, such as Chianti and APEC. Cavalcanti et al. 19] calculated the weighted oscillator strengths $(g f)$ using optimized electrostatic parameters for transitions among levels of the configurations, namely $2 s^{2} 2 p, 2 p^{3}, 2 s^{2} 3 l, 2 s 2 p 3 l, 2 p^{2} 3 l, 2 s^{2} 4 l, 2 s 2 p 4 p, 2 s 2 p 4 d, 2 s^{2} 5 d, 2 s 2 p 5 p$ and $2 s 2 p 5 d$. In our study, we calculated the $g f$ and radiative rates for transitions among 320 levels. By including other type transitions such as M1, E2 and M2, the number of transitions increases by orders of magnitude. These lost features in the astrophysical modelling codes might explain the disagreement of fitting in astrophysical X-ray spectral analyses. The results of $g f$ and radiative rates $A$ are listed in Table 11, along with effective collision strengths which will be discussed in the next section.

In Table 5 we compared our $g f$-values with other different predictions for some transitions. Fig. 3 visually exhibits such comparison for all available transitions. Here we pay special attention on the comparison with the data used by the Chianti code. For most strong transitions, our results agree with predictions of Zhang \& Sampson [16] within 20\%. Fig.3-(a) clearly reveals that our results are systematically higher than predictions used by the Chianti. However, three strong transitions of $2 s 2 p^{2}{ }^{4} P_{3 / 2}-2 s 2 p\left({ }^{3} P\right) 3 d^{2} D_{5 / 2}(4-48), 2 s 2 p^{2}{ }^{2} D_{3 / 2}-2 s 2 p\left({ }^{3} P\right) 3 d{ }^{4} D_{5 / 2}(7-45)$ and $2 p^{3}{ }^{2} P_{3 / 2}-2 p^{2}\left({ }^{3} P\right) 3 d^{4} D_{3 / 2}(15-44)$, display discrepancies being up to a factor of $\sim 4$. By gradually decreasing the configurations, we confirm that the large differences are mainly resulted from the CI effect of the $2 p^{3}$ configuration. When only configurations of $2 s^{2} 2 p, 2 s 2 p^{2}, 2 s^{2} 3 l$ and $2 s 2 p 3 l$ are considered, the $g f$ increase from 0.085 and 0.054 to 0.404 and 0.126 for the $2 s 2 p^{2}{ }^{4} P_{3 / 2}-2 s 2 p\left({ }^{3} P\right) 3 d^{2} D_{5 / 2}(4-48)$ and $2 s 2 p^{2}{ }^{2} D_{3 / 2}-2 s 2 p\left({ }^{3} P\right) 3 d^{4} D_{5 / 2}(7-45)$ transitions, respectively, they show a good agreement with the values of the Chianti. But the $g f$ rapidly decrease to 0.117 and 0.052 again, when the $2 p^{3}$ configuration has been included. For the $2 p^{3}{ }^{2} P_{3 / 2}-2 p^{2}\left({ }^{3} P\right) 3 d^{4} D_{3 / 2}(15-44)$ transition, the large difference is due to the CI effect among the $n=3$ complexes. When only the $2 p^{2} 3 d$ configuration of the $n=3$ complexes has been included, the $g f$ is 0.1136 which agrees well with the adopted data (0.1099) by the Chianti. When another two related $3 d$-complexes such as $3 s^{2} 3 d$ and $2 s 2 p 3 d$ have been included, the value increases to 0.1225. The $g f$ value steadily increases to 0.1978 when other $n=3$ complexes have been included. Therefore, the large differences between present results and other different theoretical predictions, are due to the CI effect. For those weak transitions $(g f<0.1)$, more transitions show large discrepancies between different calculations as shown in Fig.3-(b). The differences are even up to an order of magnitude for a few transitions, which leaves a scope of improvement. 

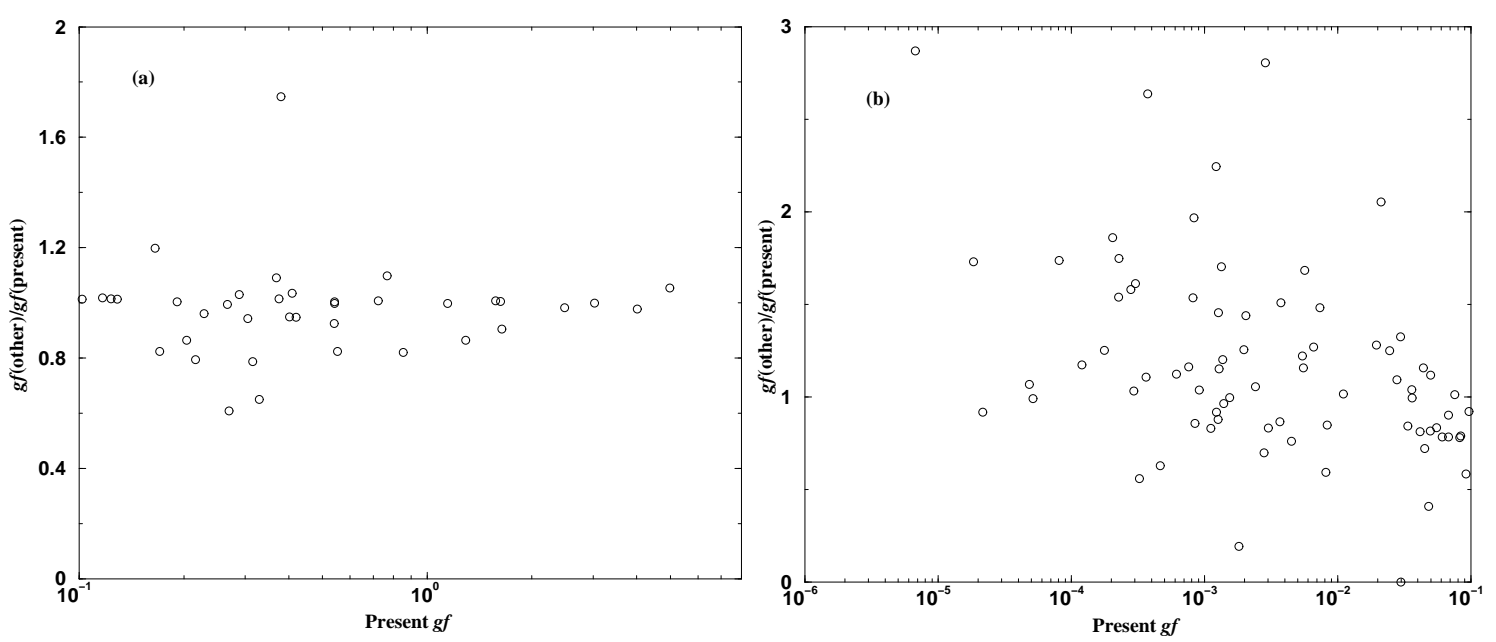

FIG. 4: Comparison of the Si XI weighted oscillator strengths from different calculations. The x-axis denotes the present results, the y-axis represents ratio of the data included by the Chianti versus present ones. (a) For strong transitions $g f>0.1$, (b) For weak transition $10^{-6}<g f<0.1$.

\subsection{Si XI}

In the Chianti database, the $g f$ of electric dipole allowed transitions of Si XI are included for transitions among 46 levels belonging to the $2 s^{2}, 2 s 2 p, 2 p^{2}, 2 s 3 l$ and $2 p 3 l$ configurations. These data was from study of Sampson et al. 20] who adopted the DFS code developed by them [24]. Coutinho et al. 22] also performed the calculation of the $g f$ by including another configurations of $2 s 4 l, 2 p 4 l, 2 s 5 l$ and $2 p 5 l$. In our study, the $g f$ and radiative rates for transitions among 350 levels were calculated. By including other type transitions such as M1, E2 and M2, the number of transitions increases by orders of magnitude relative to available data. The radiative decay rates to higher excited levels such as levels of $2 l 3 l^{\prime}$ configurations, have also been obtained. These data could be used to investigate cascade effects to higher levels, which may be important in the high-density laser-produced plasma. The results of $g f$ and radiative rates $A$ are listed in Table 12 .

In Table 6 we compare our results with the data used by the Chianti code and the results of Coutinho \& Trigueiros 22 for some transitions. A visual comparison for all available transitions is illustrated by Fig.4. For strong transitions $(g f>0.1)$, present predictions agree with the data used by Chianti code within $\sim 20 \%$. But three transitions such as $2 s^{2}{ }^{1} S_{0}-2 s 3 p{ }^{3} P_{1}$ (1-15), $2 s 2 p{ }^{1} P_{1}-2 p 3 p{ }^{1} S_{0}$ (5-25) and $2 s 2 p{ }^{1} P_{1}-2 p 3 p{ }^{3} P_{0}$ (5-44), differ up to $\sim 70 \%$. By gradually decreasing the configurations, we find that the large difference is still present for the $2 s^{2}{ }^{1} S_{0^{-}}$ $2 s 3{ }^{3} P_{1}(1-15)$ transition, and our prediction is always significantly lower than the value used by the Chianti when the $2 p 3 l$ complexes are included. However, the $g f$ becomes being higher than the later case by a factor of $\sim 5$ without the $2 p 3 l$ complexes. In the work of Sampson et al. [20], they explain that the large difference of collision strength $\Omega$ to be a level mixing of ${ }^{1} P_{1}$ and ${ }^{3} P_{1}$. Whether the same behavior occurs for the weighted oscillator strength $g f$. We found that the sum $g f$ of the two transitions $2 s^{2}{ }^{1} S_{0}-2 s 3 p{ }^{1} P_{1}(1-13)$ and $2 s^{2}{ }^{1} S_{0}-2 s 3 p{ }^{3} P_{1}(1-15)$, are 0.6068 and 0.6130 for above two cases, and this agree with the sum $g f(0.5642)$ of Chianti within $10 \%$. In this study, the sum $g f$ is 0.5892 , which also agrees with that of Chianti. Therefore the level mixing and CI maybe the possible reasons of the large differences. For those weak transitions $(g f<0.1)$, more transitions show large discrepancies between different calculations as shown in Fig.4-(b). The large differences may be from the CI effect without considered, which leaves a scope of improvement.

\section{COLLISION STRENGTH $\Omega$ AND EFFECTIVE COLLISION STRENGTH $\Upsilon$}

In this study, a self-consistent electron impact collision strengths $\Omega$ has been calculated at scattered electron energies ranging from 4 to $10000 \mathrm{eV}$ being cover the entire energy range. In Table 7-9, we list the $\Omega$ at ten scattered electron energy points such as 10, 50, 100, 200, 400, 600, 800, 1000, 1500 and $2000 \mathrm{eV}$. In practical applications, effective collision strengths appear more important. Assuming a Maxwellian distribution for the thermal electrons, 

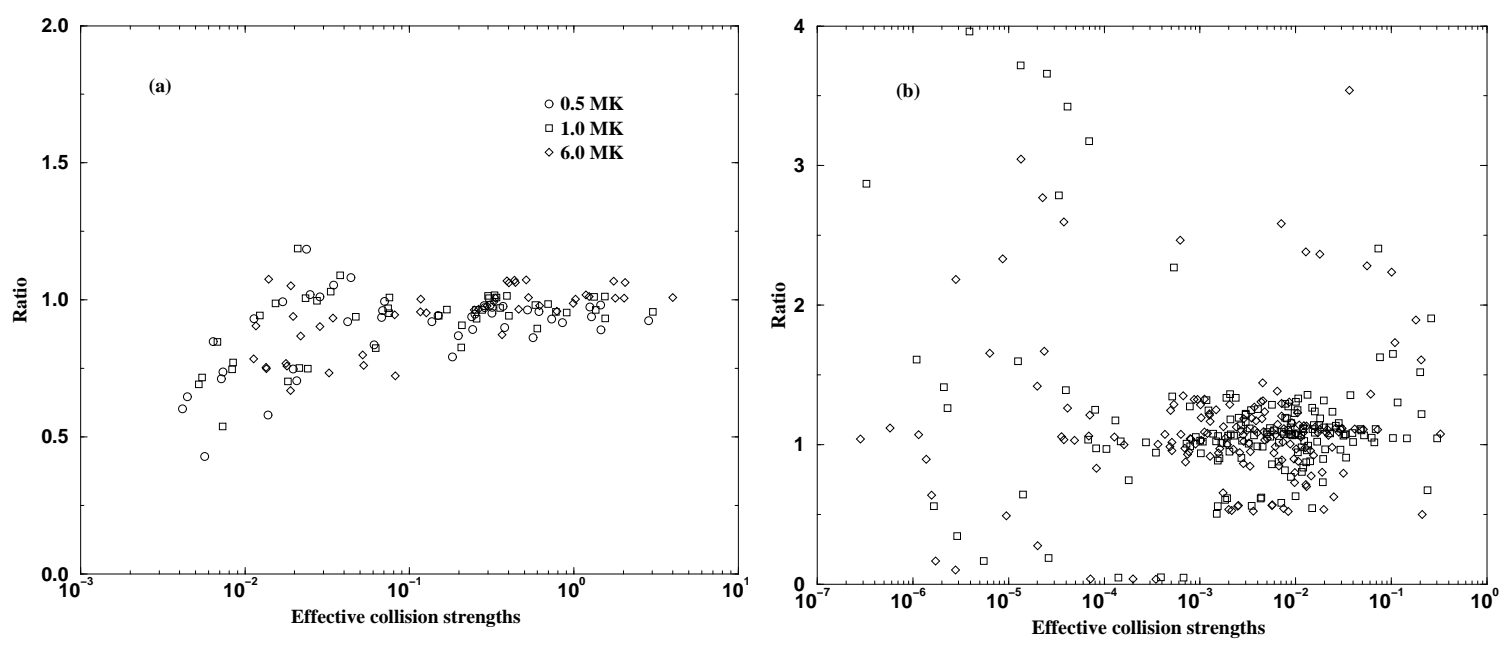

FIG. 5: Comparison of the effective collision strengths $\Upsilon$ of Si IX for different calculations. The x-axis denotes the present $\Upsilon$, the y-axis denotes ratio of the data used by the Chianti code versus present ones. (a) allowed transitions, while (b) other type transitions.

the effective collision strengths are derived through numerical integration,

$$
\Upsilon_{i j}=\int_{0}^{\infty} \Omega_{i j} \exp \left(-\frac{E}{k T_{e}}\right) d\left(\frac{E}{k T_{e}}\right),
$$

where $E$ is the scattered electron energy, $k$ is the Boltzmann constant, and $T_{e}$ is the electron temperature. Correspondingly, the excitation rate coefficients $\left(\right.$ in $\mathrm{cm}^{3} \mathrm{~s}^{-1}$ ) used in the statistical equilibrium to derive the level populations, can be obtained directly from the effective collision strength

$$
C_{i j}=\frac{8.63 \times 10^{-6}}{\omega_{i} T_{e}^{1 / 2}} \exp \left(\frac{-E_{i j}}{k T_{e}}\right) \Upsilon_{i j}
$$

where $E_{i j}$ is the energy difference between levels $i$ and $j$, and $\omega_{i}$ is the statistical weight of level $i$. In the following, we present our results of (effective) collision strengths for each ions in detail, and make an assessment through comparison with published data.

\subsection{Si IX}

In the Chianti database, only limited excitation data among the lowest 46 levels is available for Si IX, and the data are taken from work of Bhatia \& Doschek 12] who reported values at three incident electron energies of 20, 40 and 60 Ryd. The work of Aggarwal \& Baluja 25] for forbidden transitions is also included. Aggarwal \& Baluja 25] adopted a more accurate approach - $R$-matrix [26] to derive the excitation data, so far, which is the best reliable data for this ion, because coupling effects among different channels have been considered. But only the excitations among levels with $n=2$ complexes were reported. A much earlier work is performance of Mason \& Bhatia 27] who calculated the excitation data among the lowest 20 levels using the distorted wave approximation (DWA). To our knowledge, the latest work of the collision strengths for carbon-like Si is results of Zhang \& Sampson [28], yet only $\Delta n=0$ DWA excitation data within $n=2$ configurations is available. Here we present a self-consistent excitation data for a large amount of transitions.

In Table 10, we present the effective collision strengths for excitations from lowest 20 levels to higher levels up to 560-th level at seven temperatures: 0.5, 1.0, 2.0, 3.0, 4.0, 5.0 and 6.0 MK. Moreover the weighted oscillator strengths $(g f)$ and radiative rates $(A)$ are listed in this table. The indices used to represent the lower and upper levels of a transition have already been defined in Table 1 .

We pay a special attention on the comparison with the data used by the Chianti code. In Table 13, the $\Upsilon$ is given for some transitions at three temperatures of $0.5,1.0$ and 6.0 MK. The comparison with all available $\Upsilon$ is shown by Fig.5. Fig.5-(a) illustrates the comparison for allowed transitions, while Fig.5-(b) displays the comparison for other type transitions. For the allowed transitions, present results of $\Upsilon$ are systematically higher than the data used by 

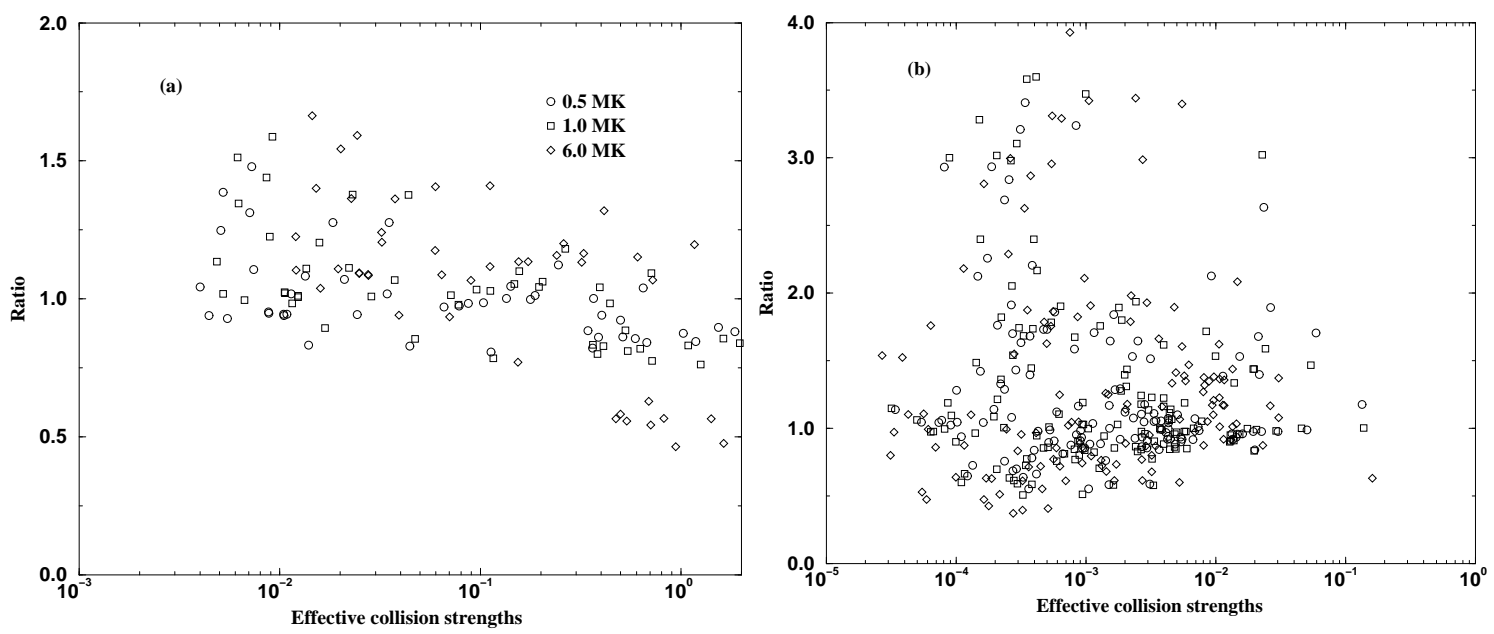

FIG. 6: Comparison of the effective collision strengths $\Upsilon$ of Si X for different calculations. The x-axis denotes the present $\Upsilon$, the $\mathrm{y}$-axis denotes ratio of the data used by the Chianti code versus present ones. (a) allowed transitions, while (b) other type transitions.

the Chianti code slightly, yet they agree within $\sim 20 \%$ except for a few excitations to the levels of $2 s^{2} 2 p 3 s$ such as $2 s^{2} 2 p^{2}{ }^{3} P_{0}-2 s^{2} 2 p 3 s^{3} P_{1}$ (1-22), $2 s^{2} 2 p^{2}{ }^{3} P_{1}-2 s^{2} 2 p 3 s^{3} P_{0}(2-21)$ and $2 s^{2} 2 p^{2}{ }^{1} D_{2}-2 s^{2} 2 p 3 s{ }^{1} P_{1}$ (4-24), in which the differences are up through to $50 \%$. For these transitions, the $g f$ differs $\sim 30 \%$ between the two different theoretical calculations. Discussions in Sect.2.1 have indicated that the CI maybe the possible reason. For forbidden transitions, we note that the discrepancies are even higher up to factors of $\sim 1.5-9$. The $\Upsilon$ of the Chianti is systematically higher than present results for excitations up through to the level of $2 p^{4}{ }^{1} S_{0}$. And the differences can reach up to $\sim 4$ times in some cases such as $2 s^{2} 2 p^{2}{ }^{3} P_{0}-2 s^{2} 2 p^{2}{ }^{3} P_{1}(1-2), 2 s^{2} 2 p^{2}{ }^{3} P_{0}-2 s^{2} 2 p^{2}{ }^{3} P_{2}(1-3), 2 s^{2} 2 p^{2}{ }^{3} P_{1}-2 s^{2} 2 p^{2}{ }^{3} P_{2}(2-3)$, etc. This is due to the the consideration of resonant excitations in the calculation of Aggarwal \& Baluja [25] who adopted the $R$-matrix method. In some forbidden transitions from much higher excited levels $(>20)$, we also note the discrepancies being up to an order of magnitude, such as $2 s 2 p^{3}{ }^{3} D_{3}-2 s^{2} 2 p 3 p{ }^{3} S_{1},{ }^{3} P_{1,2}(7-29,31,32)$. Such differences follow the discrepancies in the relevant $g f$, in which the CI effect gives rise to the large differences. However for most forbidden excitations, present $\Upsilon$ is in agreement with the data used by the Chianti code. This discussion indicates that there is a scope of improvement by consideration of resonant effect and much more CI effect.

\subsection{Si X}

So far, only two literatures reported $\Omega$ and/or $\Upsilon$ of $n=2-3$ excitations, one is the work of Zhang \& Sampson 16] using relativistic DW approximation, the other is an unpublished calculation of Sampson \& Zhang [18]. The two calculations are extensively used by present astrophysical modelling. Zhang et al. [17] had adopted $R$-matrix approach to calculate the $\Omega$, whereas their calculation is confined to transitions among 15 fine-structure levels belonging to 8 LS terms $2 s^{2} 2 p\left({ }^{2} P_{1 / 2,3 / 2}^{0}\right), 2 s 2 p^{2}\left({ }^{4} P_{1 / 2,3 / 2,5 / 2},{ }^{2} D_{3 / 2,5 / 2},{ }^{2} S_{1 / 2},{ }^{2} P_{1 / 2,3 / 2}\right), 2 p^{3}\left({ }^{4} S_{3 / 2}^{0},{ }^{2} D_{3 / 2,5 / 2}^{0},{ }^{2} P_{1 / 2,3 / 2}^{0}\right)$, and only partial data was reported. Recently, Keenan et al. 29] re-calculated these data again using $R$-matrix method, and listed all these data at much finner temperature grids. These excitation data was widely used by current astrophysical modelling codes such as Chianti, MEKAL and APEC codes. However a poor modelling for emissions of highly charged $\mathrm{Si}$ in the astrophysical spectral analysis, is still existed $[3,[30]$. As stated in these literatures, uncertainties of the excitation data is the main possible source of the poor modelling. In our present work, a self-consistent calculation is reported for the collision strength at ten scattered electron energies as listed in Table 8. Assuming the Maxwellian energy distribution, averaged collision strengths $(\Upsilon)$ at seven temperatures are listed in Table 11 for excitations from lowest 15 levels. The indices used to represent the lower and upper levels for a given transition have already been defined in Table 2.

In Table 14, we compare our $\Upsilon$ with the data used by the Chianti code at three temperatures of 0.5, 1.0 and 6.0 MK, which are typical temperatures of stellar X-ray emitters. The comparison with all available $\Upsilon$ is illustrated in Fig.6. Fig.6-(a) represents the allowed transitions, while Fig.6-(b) shows the comparison for other type transitions. For most allowed transitions, present results of $\Upsilon$ agree with the data included in the Chianti data sets within $20 \%$, yet 

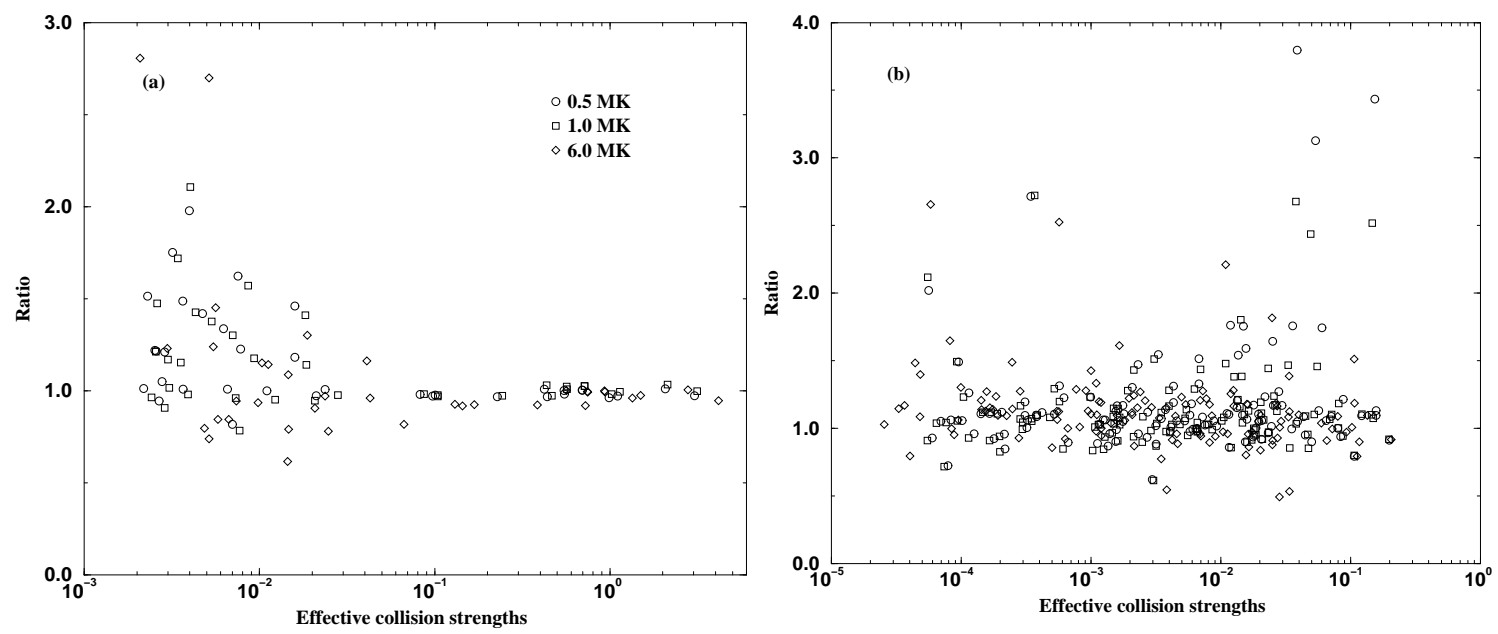

FIG. 7: Comparison of the effective collision strengths $\Upsilon$ of Si XI for different calculations. The x-axis denotes the present $\Upsilon$, the $\mathrm{y}$-axis denotes ratio of the data used by the Chianti code versus present ones. (a) allowed transitions, while (b) other type transitions.

differences up to $50 \%$ are also apparent, such as excitations of $2 s^{2} 2 p^{2} P_{1 / 2}-2 s 2 p^{2}{ }^{2} D_{3 / 2}(1-7), 2 s^{2} 2 p^{2} P_{3 / 2}-2 s 2 p^{2}{ }^{2} D_{5 / 2}$ $(2-6)$, etc. We found that the large differences occur for excitations up through to the level of $2 s 2 p^{3}{ }^{2} P_{3 / 2}$. And at higher temperature, the differences is more clear. In the Chianti database, the work of Zhang et al. [17] is used for these excitations, who adopted $R$-matrix method by accounting for the resonant effect. So we believe the large differences are resulted from the resonant effect. For those forbidden transitions, more excitations exhibit large differences. And in some cases, the discrepancies are up through to factors of $\sim 4$. Such phenomena is natural, because the resonant excitations generally is more obvious. Fox example, $2 s^{2} 2 p^{2} P_{3 / 2}-2 s 2 p^{2}{ }^{4} P_{1 / 2}(2-3)$, the result of Zhang et al. 17] is higher than present one by a factor of $\sim 2$. While for an allowed transition of $2 s^{2} 2 p{ }^{2} P_{1 / 2}-2 s 2 p^{2}{ }^{2} S_{1 / 2}$ (1-8), the difference is less than 20\%. In addition, the CI effect is another important reason for the large differences, such as for $2 s 2 p^{2}{ }^{4} P_{3 / 2}-2 s 2 p\left({ }^{3} P\right) 3 d^{2} D_{5 / 2}(4-48)$ and $2 s 2 p^{2}{ }^{4} P_{3 / 2}-2 p^{2}\left({ }^{3} P\right) 3 s^{2} P_{1 / 2}(4-70)$. However, such differences follow the discrepancies of the relevant $g f$. In the Sect.2.2, we distinguish the large differences are from the CI effect of the configuration $2 p^{3}$. This comparison also indicates that the improvement by considering the resonance excitation and more CI effect, is very necessary.

\subsection{Si XI}

For this ion, only limited electron impact excitation data among the lowest 46 levels is available so far, and all excitation data was calculated using DW approximation. Yet most available $\Omega$ is confined to transitions with $\Delta n=0$. To our best knowledge, only one work performed the calculation for $\Delta n=1$ transitions, and it is a work about two decades ago 20. These data is still used by the Chianti code. In this study, self-consistent results of $\Omega$ and $\Upsilon$ of excitations from lowest 10 levels, are reported for Si XI as shown in Table 9 and 12 respectively. The reported $\Upsilon$ covers the typical coronal temperature range of $\sim 0.1-10 \mathrm{MK}$. The indices used in tables to represent the lower and upper levels of a transition have already been defined in Table 3.

In Table 15, a comparison of our $\Upsilon$ with the data used by the Chianti code at three temperatures is given for partial transitions. The comparison with all available $\Upsilon$ is also illustrated through Fig.7. Fig.7-(a) illustrates the comparison for allowed transitions at three temperatures of 0.5, 1.0 and 6.0 MK, while Fig.7-(b) shows the comparison for other type transitions. For the allowed transitions, the two different calculations show a better agreement except for a few transitions such as $2 s^{2}{ }^{1} S_{0}-2 p 3 s{ }^{3} P_{1},{ }^{1} P_{1}(1-22,24)$, and $2 s^{2}{ }^{1} S_{0}-2 p 3 d^{3} D_{1}(1-38)$. In these cases, the differences are up to a factor of $\sim 4$, and they follow the differences in $g f$. The discussion in Sect.2.3 indicates the discrepancies in $g f$ are due to the level mixing and CI effect. For other type transitions, a majority of transitions shows a good agreement at temperature of $\sim 0.1-6 \mathrm{MK}$, whereas large differences are also present for some transitions. The interpretation for the large discrepancies can be divided into two group, one is due to the different data sources in the Chianti data sets. For excitations among the low-lying 10 levels, the work of Berrington et al. 31] is used, who considered the resonant effect by using the $R$-matrix approach. So the $\Upsilon$ of the Chianti is larger than present ones by factors of up through to $\sim 3$. The other possible reason maybe from the different inclusions of CI, such as for $2 s^{2}{ }^{1} S_{0}-2 p 3 s{ }^{3} P_{1}(1-22)$. 
The difference also follows the discrepancy in relevant $g f$. This leaves a scope of improvement by considerations of resonance effect and much more CI effect.

\section{CONCLUSIONS}

In this work, radiative rates and oscillator strengths $g f$ among 560, 320 and 350 levels of Si IX, Si X and Si XI are reported, respectively. A self-consistent calculation of (effective) collision strengths is performed with large CI using the fully relativistic FAC code of $\mathrm{Gu}[\underline{8}$. In general, our energy levels agree with the available experimental values within $2 \%$. For strong transitions $(g f>0.1)$, present results of radiative rates or weighted oscillator strengths show a good agreement with available data except for a few transitions with discrepancies of several times. By gradually decreasing the CI, we found that the relatively large differences can be diminished, which indicates CI plays an important role in accurate determinations of $g f$. For weak transitions $(g f<0.1)$, the CI effect appears more obvious. And the discrepancies can reach up to an order of magnitude in some cases.

Self-consistent collision strengths and effective collision strengths by considering large CI are also reported in this study. For allowed transitions, present work shows a better agreement with available data except for a few excitations, in which the differences are up to factor of $\sim 3$. Such differences follow the discrepancies of $g f$, which reveals the large differences maybe from the CI effects. Though the present $\Upsilon$ agree with the data of the Chianti within $20 \%$ for a majority of forbidden transitions, a certain of excitations show large differences up to $\sim 4$, which can be attributed to the resonant effect and CI effect in the different calculations, because the excitation data among levels of $n=2$

complexes in the Chianti data sets was from $R$-matrix method. The discussion also implies that there is a scope of improvement by considering the resonant effect and more CI effect.

\section{ACKNOWLEDGMENTS}

This work was supported by the National Natural Science Foundation under Grant No. 10433010, No. 10373014 and No. 10403007. This work is also financed by Chinese Academy of Sciences under Grant No. KJCX2-W2.

[1] A.J.J. Raassen, R. Mewe, M. Audard, et al., Astron. Astrophys. 389 (2002) 228

[2] A.J.J. Raassen, J.-U. Ness, R. Mewe, et al., Astron. Astrophys. 400 (2003) 671

[3] M. Audard, E. Behar, M. Güdel, et al., Astron. Astrophys. 365 (2001) L329

[4] K.M. Aggarwal, F.P. Keenan, S. Nakazaki, Astron. Astrophys. 436 (2005) 1141

[5] G.Y. Liang, G.X. Dong, J. L. Zeng, At. Data Nucl. Data Tables 88 (2004) 83

[6] E. Landi, A.K. Bhatia, At. Data Nucl. Data Tables 92 (2006) 305

[7] G.Y. Liang, J.Y. Zhong, Z. Jin, et al., (2006) (in preparation)

[8] M.F. Gu, Astronphys. J. 582 (2003) 1241

[9] M.F. Gu, Astronphys. J. Suppl. 156 (2005) 105

[10] K. G. Dyall, I. P. Grant, C. T. Johnson, et al., Comput. Phys. Commun. 55 (1989) 424

[11] K.M. Aggarwal, Astrophys. J. Supp. 118 (1998) 589

[12] A.K. Bhatia, G.A. Doschek, At. Data Nucl. Data Tables 55 (1993) 281

[13] R.V. Orloski, A.G. Trigueiros, G.H. Cavalcanti, J. Quant. Spectrosc. Radiat. Transfer 61 (1999) 665

[14] L.J. Radziemski, V. Kaufman, J. Opt. Soc. Am. 59 (1969) 424

[15] B. Edlen, Phys. Scripta 28 (1983) 483

[16] H.L. Zhang, D.H. Sampson, At. Data Nucl. Data Tables 58 (1994) 255

[17] H.L. Zhang, M. Graziani, A.K. Prahan, Astron. Astrophys. 283 (1994) 319

[18] D.H. Sampson, H.L. Zhang, unpublished calculation (1995)

[19] G.H. Cavalcanti, F.R.T. Luna, A.G. Trigueiros, J. Quant. Spectrosc. Radiat. Transfer 64 (2000) 5

[20] D.H. Sampson, S.J. Goett, R.E.H. Clark, At. Data Nucl. Data Tables 30 (1984) 125

[21] H.L. Zhang, D.H. Sampson, At. Data Nucl. Data Tables 52 (1992) 143

[22] L. H. Coutinho, A.G. Trigueiros, J. Quant. Spectrosc. Radiat. Transfer 68 (2001) 643

[23] R. D. Cowan, The theory of atomic structure and spectra, Berkeley, CA: University of California Press (1981)

[24] D.H. Sampson, H.L. Zhang, A. K. Mohanty, R.E.H. Clark, Phys. Rev. A 40 (1989) 604

[25] K.M. Aggarwal, K.L. Baluja J. Phys. B 16 (1983) 107

[26] A. Hibbert, Comput. Phys. Commun. 9 (1975) 141

[27] H.E. Mason, A.K. Bhatia, Mon. Not. Royal Astron. Soc. 184 (1978) 423

[28] H.L. Zhang, D.H. Sampson, At. Data Nucl. Data Tables 63 (1996) 275 
TABLE IV: Comparison of weighted oscillator strength $g f_{j i}$ (written in form a \pm b implying a $\times 10^{ \pm b}$ ) for some transitions of Si IX. $i$ and $j$ are denoted in Table I. OTC99 represents the work of Orloski et al. [13].

\begin{tabular}{|c|c|c|c|c|c|c|c|c|c|c|c|}
\hline $\bar{i}$ & $j$ & FAC & CIV46 & Chianti & OTC99 & $\bar{i}$ & $\bar{j}$ & $\overline{\mathrm{FAC}}$ & CIV46 & Chianti & $\overline{\text { OTC99 }}$ \\
\hline 1 & 9 & $8.160-2$ & $7.797-2$ & $8.310-2$ & $7.797-2$ & 3 & 38 & $1.251-2$ & $1.301-2$ & $9.310-3$ & $1.301-2$ \\
\hline 1 & 11 & $7.759-2$ & $7.444-2$ & $8.260-2$ & $7.444-2$ & 3 & 39 & $4.623-2$ & $4.170-2$ & $3.930-2$ & $4.170-2$ \\
\hline 1 & 14 & $9.369-2$ & $9.154-2$ & $9.750-2$ & $9.154-2$ & 3 & 40 & $5.010-3$ & $4.274-3$ & $7.330-3$ & $4.274-3$ \\
\hline 1 & 22 & $6.791-2$ & $6.494-2$ & $5.360-2$ & $6.494-2$ & 3 & 42 & $6.889-4$ & $1.315-3$ & $1.280-3$ & $1.315-3$ \\
\hline 1 & 24 & $6.702-4$ & $6.514-4$ & $7.840-4$ & $6.514-4$ & 3 & 43 & $7.678-2$ & $6.860-2$ & $6.970-2$ & $6.860-2$ \\
\hline 1 & 42 & $1.061+0$ & $1.076+0$ & $1.040+0$ & $1.076+0$ & 3 & 44 & $3.494+0$ & $3.566+0$ & $3.490+0$ & $3.566+0$ \\
\hline 1 & 46 & $6.041-2$ & $7.154-2$ & $6.120-2$ & $7.154-2$ & 3 & 46 & $3.925-1$ & $4.024-1$ & $3.780-1$ & $4.024-1$ \\
\hline 1 & 51 & $3.298-3$ & $1.901-3$ & $2.290-3$ & $1.901-3$ & 3 & 51 & $1.159-4$ & $4.620-5$ & $4.830-5$ & $4.620-5$ \\
\hline 2 & 6 & $1.950-5$ & $1.947-5$ & $2.230-5$ & $1.947-5$ & 3 & 52 & $5.868-4$ & $6.265-4$ & $5.840-4$ & $6.265-4$ \\
\hline 2 & 8 & $1.805-1$ & $1.726-1$ & $1.840-1$ & $1.726-1$ & 4 & 7 & $8.741-4$ & $8.480-4$ & $9.700-4$ & $8.480-4$ \\
\hline 2 & 9 & $4.883-2$ & $4.707-2$ & $4.870-2$ & $4.707-2$ & 4 & 10 & $9.522-5$ & $5.560-5$ & & $5.560-5$ \\
\hline 2 & 13 & $1.066-4$ & $1.080-4$ & $1.130-4$ & $1.080-4$ & 4 & 11 & $3.012-4$ & $2.822-4$ & $3.450-4$ & $2.822-4$ \\
\hline 2 & 15 & $1.274-3$ & $1.122-3$ & $1.680-3$ & $1.122-3$ & 4 & 14 & $1.408-4$ & $8.050-5$ & $3.670-4$ & $8.050-5$ \\
\hline 2 & 21 & $6.865-2$ & $6.579-2$ & $5.500-2$ & $6.579-2$ & 4 & 23 & $1.053-3$ & $1.022-3$ & $9.750-4$ & $1.022-3$ \\
\hline 2 & 38 & $2.601-3$ & $5.754-4$ & $2.710-3$ & $5.754-4$ & 4 & 39 & $1.070-2$ & $8.760-3$ & $9.490-3$ & $8.760-3$ \\
\hline 2 & 40 & $4.477-2$ & $3.726-2$ & $3.790-2$ & $3.726-2$ & 4 & 42 & $7.740-4$ & $7.310-4$ & $4.650-4$ & $7.310-4$ \\
\hline 2 & 42 & $3.934-1$ & $4.158-1$ & $4.040-1$ & $4.158-1$ & 4 & 46 & $9.187-4$ & $9.190-4$ & $1.080-3$ & $9.190-4$ \\
\hline 2 & 47 & $2.831-1$ & $2.905-1$ & $2.700-1$ & $2.905-1$ & 5 & 11 & $1.567-4$ & $1.372-4$ & $2.150-4$ & $1.372-4$ \\
\hline 2 & 51 & $2.416-3$ & $1.601-3$ & $1.510-3$ & $1.601-3$ & 5 & 14 & $1.931-4$ & $1.709-4$ & $2.560-4$ & $1.709-4$ \\
\hline 3 & 6 & $4.506-5$ & $4.774-5$ & $5.950-5$ & $4.774-5$ & 5 & 15 & $1.808-1$ & $1.757-1$ & $1.820-1$ & $1.757-1$ \\
\hline 3 & 13 & $2.115-3$ & $1.948-3$ & $2.330-3$ & $1.948-3$ & 5 & 22 & $1.009-3$ & $8.839-4$ & $9.140-4$ & $8.839-4$ \\
\hline 3 & 14 & $4.928-1$ & $4.828-1$ & $5.130-1$ & $4.828-1$ & 5 & 24 & $8.495-2$ & $7.945-2$ & $6.500-2$ & $7.945-2$ \\
\hline 3 & 22 & $8.687-2$ & $8.330-2$ & $6.990-2$ & $8.330-2$ & 5 & 42 & $4.260-3$ & $2.513-3$ & $1.730-3$ & $2.513-3$ \\
\hline 3 & 23 & $2.574-1$ & $2.465-1$ & $2.050-1$ & $2.465-1$ & 5 & 46 & $7.403-4$ & $6.538-4$ & $5.470-4$ & $6.538-4$ \\
\hline 3 & 24 & $2.782-4$ & $2.357-4$ & $3.540-4$ & $2.357-4$ & 5 & 51 & $1.224+0$ & $1.254+0$ & $1.120+0$ & $1.254+0$ \\
\hline
\end{tabular}

TABLE V: Comparison of weight oscillator strength $(g f)$ for some transitions of Si X. CLT00 refers to the work of Cavalcanti et al. [19].

\begin{tabular}{rrrrrrrrrr}
\hline \hline$i$ & $j$ & FAC & CLT00 & Chianti & $i$ & $j$ & FAC & CLT00 & Chianti \\
\hline 1 & 7 & $1.421-1$ & & $1.360-1$ & 4 & 21 & $1.500-1$ & $1.134-1$ \\
1 & 8 & $1.126-1$ & & $1.110-1$ & 4 & 45 & $2.807+0$ & $2.380+0$ & $2.316+0$ \\
1 & 9 & $1.900-1$ & $1.840-1$ & $1.750-1$ & 4 & 53 & $8.366-4$ & $2.357-4$ \\
1 & 16 & $5.113-2$ & & $4.013-2$ & 4 & 56 & $5.504-4$ & $3.396-4$ \\
1 & 19 & $1.246+0$ & $1.300+0$ & $1.124+0$ & 4 & 74 & $1.172-3$ & $9.430-4$ \\
1 & 28 & $1.200-1$ & $1.870-1$ & $8.156-2$ & 4 & 82 & $5.016-2$ & $4.633-2$ \\
1 & 59 & $5.940-4$ & & $1.661-3$ & 5 & 25 & $5.520-5$ & $8.375-5$ \\
1 & 144 & $4.307-2$ & $8.930-2$ & & 5 & 45 & $2.558-1$ & $6.400-3$ & $1.900+0$ \\
2 & 6 & $2.373-1$ & $2.320-1$ & $2.040-2$ & 5 & 49 & $1.575+0$ & $1.606+0$ \\
2 & 9 & $1.545-1$ & $1.570-1$ & $1.630-1$ & 5 & 66 & $3.527-4$ & $3.698-4$ \\
2 & 10 & $5.872-1$ & $5.820-1$ & $5.800-1$ & 5 & 84 & $7.944-4$ & $9.566-4$ \\
2 & 20 & $2.240+0$ & $2.330+0$ & $2.011+0$ & 7 & 12 & $4.551-2$ & $4.430-2$ \\
2 & 192 & $1.928-1$ & $1.880-1$ & & 7 & 13 & $4.547-1$ & $4.580-1$ \\
3 & 21 & $3.023-2$ & & $2.279-2$ & 7 & 52 & $8.013-2$ & $1.500-2$ \\
3 & 38 & $4.486-3$ & & $4.188-3$ & 7 & 54 & $2.256+0$ & $2.120+0$ & $6.690-1$ \\
3 & 44 & $1.258+0$ & $1.300+0$ & $1.091+0$ & 8 & 12 & $7.510-3$ & $7.800-1$ \\
3 & 51 & $3.880-2$ & & $3.795-2$ & 8 & 53 & $1.689-1$ & $2.136+0$ \\
3 & 74 & $4.216-4$ & & $5.116-4$ & 8 & 107 & $6.846-3$ & $1.171-1$ \\
\hline \hline
\end{tabular}

[29] F.P. Keenan, E. O'Shea, R.J. Thomas, et al., Mon. Not. Royal Astron. Soc. 315 (2000) 450

[30] M. Güdel, M. Audard, K. Briggs, et al., Astron. Astrophys. 365 (2001) L336

[31] K.A. Berrington, P.G. Burke, P.L. Dufton, A.E. Kingston, At. Data Nucl. Data Tables, 33 (1985) 195 
TABLE VI: Comparison of weight oscillator strength $(g f)$ for some transitions of Si XI. CT01 refers to the work of Coutinho \& Trigueiros [22].

\begin{tabular}{rrrrrrrrr}
\hline \hline$i$ & $j$ & FAC & CT01 & Chianti & $i$ & $j$ & FAC & CT01 \\
\hline 1 & 5 & $2.666-1$ & & $2.652-1$ & 4 & 8 & $3.755-1$ & $4.010-1$ \\
1 & 13 & $5.533-1$ & & $4.557-1$ & 4 & 11 & $1.702-1$ & $1.620-1$ \\
1 & 15 & $3.592-2$ & & $1.085-1$ & 4 & 17 & $3.620-2$ & $1.403-1$ \\
1 & 22 & $3.744-4$ & & $9.865-4$ & 4 & 18 & $5.418-1$ & $3.607-2$ \\
2 & 7 & $1.020-1$ & $1.090-1$ & $1.034-1$ & 4 & 31 & $1.651-1$ & $1.730-1$ \\
2 & 17 & $7.234-1$ & $7.120-1$ & $7.291-1$ & 4 & 32 & $3.683-1$ & $3.730-1$ \\
2 & 29 & $4.403-2$ & & $5.090-2$ & 4 & 73 & $1.180-1$ & $1.100-1$ \\
2 & 53 & $1.342-1$ & $1.300-1$ & & 4 & 68 & $1.623-1$ & $1.590-1$ \\
3 & 7 & $7.572-2$ & $8.080-2$ & $7.679-2$ & 4 & 107 & $2.096-1$ & $1.970-1$ \\
3 & 8 & $1.286-1$ & $1.370-1$ & $1.304-1$ & 4 & 125 & $1.209-1$ & $1.040-1$ \\
3 & 17 & $5.423-1$ & & $5.446-1$ & 5 & 9 & $2.881-1$ & $3.050-1$ \\
3 & 18 & $1.625+0$ & $1.600+0$ & $1.634+0$ & 5 & 10 & $1.913-1$ & $2.340-1$ \\
3 & 27 & $2.283-1$ & $2.380-1$ & & 5 & 12 & $4.144-2$ & $2.969-1$ \\
3 & 29 & $1.166-1$ & $1.200-1$ & $1.187-1$ & 5 & 20 & $1.638+0$ & $1.670+0$ \\
3 & 32 & $9.849-2$ & $1.000-1$ & $1.046-1$ & 5 & 37 & $7.681-1$ & $7.390-1$ \\
3 & 37 & $1.226-3$ & & $1.128-3$ & 5 & 44 & $8.528-2$ & $8.600-1$ \\
3 & 54 & $3.016-1$ & $2.930-1$ & & 5 & 56 & $3.629-1$ & $3.620-1$ \\
3 & 106 & $1.127-1$ & $1.060-1$ & & 5 & 77 & $1.622-1$ & 1 \\
4 & 7 & $1.235-1$ & $1.320-1$ & $1.254-1$ & 5 & 108 & $1.543-1$ & $1.600-1$ \\
\hline \hline
\end{tabular}

TABLE XIII: Comparison of effective collision strengths $\Upsilon_{i j}$ (written in form a $\pm \mathrm{b}$ implying $\mathrm{a} \times 10^{ \pm b}$ ) of Si IX between present results and those used by the Chianti code. The data at three temperatures of $0.5,1.0$ and $6.0 \mathrm{MK}$, is listed for some transitions. For each transition, the first row represents present results and the second row represents data included in the Chianti database.

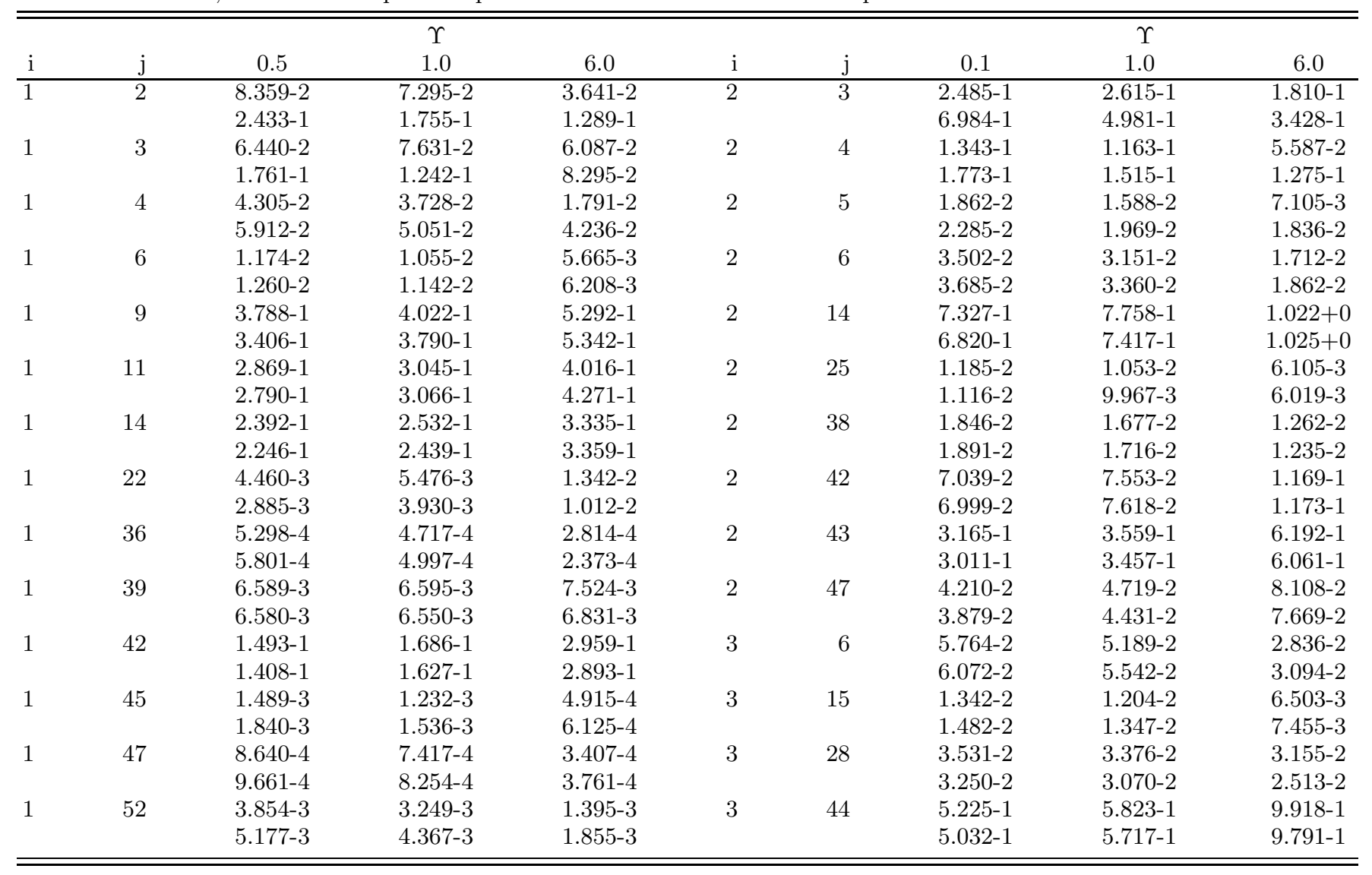


TABLE XIV: Comparison of effective collision strength $\Upsilon_{i j}$ for some excitations of Si X. Same as Table 13

\begin{tabular}{|c|c|c|c|c|c|c|c|c|c|}
\hline \multicolumn{7}{|c|}{$\Upsilon$} & \multicolumn{3}{|c|}{$\Upsilon$} \\
\hline $\mathrm{i}$ & $\mathrm{j}$ & 0.5 & 1.0 & 6.0 & $\mathrm{i}$ & $\mathrm{j}$ & 0.1 & 1.0 & 6.0 \\
\hline \multirow[t]{2}{*}{1} & $\frac{J}{2}$ & $1.899-1$ & $1.966-1$ & $1.400-1$ & 1 & 122 & $2.075-4$ & $2.221-4$ & $3.524-4$ \\
\hline & & 7.373-1 & 4.944-1 & 1.661-1 & & & $3.660-4$ & $4.046-4$ & 6.604-4 \\
\hline \multirow[t]{2}{*}{1} & 4 & $2.130-2$ & $1.936-2$ & $1.074-2$ & 2 & 5 & $5.928-2$ & $5.399-2$ & $3.054-2$ \\
\hline & & $3.578-2$ & $2.788-2$ & $1.466-2$ & & & $1.011-1$ & $7.918-2$ & 4.192-2 \\
\hline \multirow[t]{2}{*}{1} & 6 & $2.356-2$ & $2.140-2$ & $1.191-2$ & 2 & 7 & $1.340-1$ & $1.378-1$ & $1.605-1$ \\
\hline & & $4.998-2$ & $3.643-2$ & $1.737-2$ & & & $1.577-1$ & $1.382-1$ & 1.013-1 \\
\hline \multirow[t]{2}{*}{1} & 7 & $6.756-1$ & 7.173-1 & $9.409-1$ & 2 & 8 & $3.630-1$ & $3.838-1$ & 4.996-1 \\
\hline & & 5.694-1 & $5.560-1$ & $4.374-1$ & & & 2.984-1 & 3.073-1 & 2.910-1 \\
\hline \multirow[t]{2}{*}{1} & 8 & $3.874-1$ & $4.101-1$ & $5.364-1$ & 2 & 10 & $1.858+0$ & $1.966+0$ & $2.576+0$ \\
\hline & & $3.336-1$ & $3.401-1$ & 2.993-1 & & & $1.637+0$ & $1.650+0$ & $1.481+0$ \\
\hline \multirow[t]{2}{*}{1} & 12 & $3.932-3$ & $4.216-3$ & $5.811-3$ & 2 & 15 & $8.334-3$ & $8.302-3$ & $8.835-3$ \\
\hline & & $6.771-3$ & $6.708-3$ & $6.038-3$ & & & $1.403-2$ & $1.386-2$ & $1.206-2$ \\
\hline \multirow[t]{2}{*}{1} & 19 & $1.872-1$ & $2.043-1$ & $3.270-1$ & 2 & 20 & 3.661-1 & 3.944-1 & $6.076-1$ \\
\hline & & $1.897-1$ & 2.169-1 & 3.811-1 & & & $3.668-1$ & 4.109-1 & $7.000-1$ \\
\hline \multirow[t]{2}{*}{1} & 20 & $3.363-2$ & $3.017-2$ & 1.963-2 & 2 & 25 & $2.093-1$ & $2.150-1$ & 2.382-1 \\
\hline & & $3.131-2$ & $2.647-2$ & $1.543-2$ & & & $1.980-1$ & 2.041-1 & $2.215-1$ \\
\hline \multirow[t]{2}{*}{1} & 24 & $1.080-1$ & $1.110-1$ & $1.232-1$ & 2 & 37 & $3.509-2$ & $4.383-2$ & 1.117-1 \\
\hline & & $1.022-1$ & $1.054-1$ & $1.146-1$ & & & $4.480-2$ & $6.035-2$ & $1.575-1$ \\
\hline \multirow[t]{2}{*}{1} & 36 & $1.838-2$ & $2.309-2$ & $5.965-2$ & 2 & 57 & $1.781-2$ & $1.956-2$ & 2.903-2 \\
\hline & & $2.347-2$ & $3.182-2$ & $8.385-2$ & & & $1.874-2$ & $2.156-2$ & $3.283-2$ \\
\hline \multirow[t]{2}{*}{1} & 47 & $1.952-2$ & $2.145-2$ & $3.175-2$ & 2 & 99 & $5.955-4$ & $6.185-4$ & $8.743-4$ \\
\hline & & $1.836-2$ & $2.114-2$ & $3.244-2$ & & & $6.689-4$ & $6.835-4$ & $9.166-4$ \\
\hline \multirow[t]{2}{*}{1} & 67 & $1.212-2$ & $1.301-2$ & $1.824-2$ & 2 & 108 & $1.869-4$ & $2.052-4$ & $3.744-4$ \\
\hline & & $9.235-3$ & $1.020-2$ & $1.492-2$ & & & $5.484-4$ & $6.194-4$ & $1.074-3$ \\
\hline \multirow[t]{2}{*}{1} & 89 & $1.738-4$ & $1.512-4$ & $7.663-5$ & 2 & 121 & $1.598-3$ & $1.743-3$ & $2.887-3$ \\
\hline & & $3.926-4$ & $4.963-4$ & $1.186-3$ & & & $3.016-3$ & $3.394-3$ & $5.676-3$ \\
\hline \multirow[t]{2}{*}{1} & 113 & 2.139-4 & $2.196-4$ & $2.605-4$ & 2 & 125 & $8.118-4$ & $7.205-4$ & $3.652-4$ \\
\hline & & $1.765-4$ & $1.779-4$ & $2.065-4$ & & & $1.622-4$ & $1.515-4$ & $1.424-4$ \\
\hline
\end{tabular}

TABLE XV: Comparison of effective collision strength $\Upsilon_{i j}$ for some excitations of Si XI. Same as in Table 13

\begin{tabular}{|c|c|c|c|c|c|c|c|c|c|}
\hline \multirow[b]{2}{*}{$\mathrm{i}$} & \multirow[b]{2}{*}{$\mathrm{j}$} & \multicolumn{3}{|c|}{$\bar{\Upsilon}$} & \multicolumn{5}{|c|}{$\bar{\Upsilon}$} \\
\hline & & 0.5 & 1.0 & 6.0 & $\mathrm{i}$ & $\mathrm{j}$ & 0.1 & 1.0 & 6.0 \\
\hline \multirow[t]{2}{*}{1} & 5 & $1.103+0$ & $1.140+0$ & $1.493+0$ & 1 & 45 & $5.110-4$ & $5.471-4$ & $8.120-4$ \\
\hline & & $1.073+0$ & $1.134+0$ & $1.458+0$ & & & $2.225-4$ & 2.344-4 & $3.140-4$ \\
\hline 1 & & $5.717-2$ & $5.847-2$ & $6.454-2$ & & & $5.589-3$ & $5.897-3$ & $8.151-3$ \\
\hline \multirow[t]{2}{*}{1} & 13 & $2.371-2$ & $2.799-2$ & $6.662-2$ & 2 & 3 & $5.363-2$ & $4.938-2$ & $2.486-2$ \\
\hline & & $2.390-2$ & $2.734-2$ & $5.449-2$ & & & $1.677-1$ & $1.203-1$ & $4.522-2$ \\
\hline \multirow[t]{2}{*}{1} & 18 & $8.802-3$ & $8.062-3$ & $3.813-3$ & 2 & 7 & $5.473-1$ & $5.658-1$ & 7.416-1 \\
\hline & & $9.285-3$ & 8.304-3 & 4.399-3 & & & $5.484-1$ & 5.795-1 & 7.403-1 \\
\hline \multirow[t]{2}{*}{1} & 19 & $1.229-2$ & $1.126-2$ & $5.327-3$ & 2 & 14 & $3.507-2$ & $3.515-2$ & $3.767-2$ \\
\hline & & $1.301-2$ & $1.163-2$ & $6.159-3$ & & & $3.172-2$ & $3.221-2$ & $3.459-2$ \\
\hline 1 & 20 & $6.490-2$ & $6.889-2$ & $1.026-1$ & 2 & 21 & $2.329-2$ & $2.351-2$ & $2.635-2$ \\
\hline \multirow[t]{2}{*}{1} & 32 & $1.014-4$ & $9.356-5$ & $4.843-5$ & 3 & 15 & $1.065-1$ & $1.065-1$ & $1.124-1$ \\
\hline & & $1.074-4$ & $9.869-5$ & $6.769-5$ & & & $8.458-2$ & 8.529-2 & $8.917-2$ \\
\hline \multirow[t]{2}{*}{1} & 36 & $4.234-4$ & $3.861-4$ & $1.736-4$ & 3 & 24 & $1.592-3$ & $1.489-3$ & $9.132-4$ \\
\hline & & $3.382-4$ & $3.011-4$ & $1.568-4$ & & & $1.858-3$ & $1.710-3$ & $1.168-3$ \\
\hline \multirow[t]{2}{*}{1} & 40 & $1.279-4$ & $1.160-4$ & $4.851-5$ & 3 & 44 & $3.091-4$ & $2.861-4$ & $1.566-4$ \\
\hline & & $1.393-4$ & $1.218-4$ & $5.895-5$ & & & $3.700-4$ & $3.343-4$ & $1.993-4$ \\
\hline
\end{tabular}




\section{EXPLANATION OF TABLES}

TALBE I. $\quad$ Energy levels (in $R y d$ ) of Si IX ion.

Index A number assigned to each level

Configuration The configuration

Expt. The data is from NIST website at http://physics.nist.gov/cgi-bin/AtData/main_asd

FAC Data from FAC code

CIV3 The work of Aggarwal 11]

OTC99 The work of Orloski et al. 13.

Chianti The work of Bhatia \& Doschek 12]

TABLE II. $\quad$ Energy levels (in $R y d$ ) of Si X ion.

Index A number assigned to each level

Configuration The configuration

FAC Calculation from FAC code

CLT00 The work of Cavalcanti et al. 19]

Chianti The theoretical data is from literatures 15, 16, 17, 18

TALBE III. $\quad$ Energy levels (in Ryd) of Si XI ion.

Index A number assigned to each level

Configuration The configuration

FAC Present 350 level calculation from FAC code

HFR The work of Coutinho \& Trigueiros [22]

Chianti The data from Sampson et al. [20] and Zhang \& Sampson [21].

TALBE VII-IX. Collision strengths $\Omega$ for Si IX, Si X and Si XI at ten scattered electron energies (in $\mathrm{eV}$ ) of 10, 50, 100, 200, 400, 600, 800, 1000, 1500 and 2000, respectively.

The data is written in the form $\mathrm{a} \pm \mathrm{b}$ implying $\mathrm{a} \times 10^{\mathrm{b}}$.

TALBE X-XII. Effective collision strengths $\Upsilon$ for Si IX, Si X and Si XI at seven temperatures (MK): 0.5, 1.0, 2.0, 3.0, 4.0, 5.0 and 6.0 along with weighted oscillator strengths $g f$ and radiative rates $A$, respectively.

The data is written in the form a \pm b implying $a \times 10^{b}$. 
TABLE I: Energy levels (in Ryd) of Si IX ion.

\begin{tabular}{|c|c|c|c|c|c|c|c|}
\hline$\overline{I n d e x}$ & Configuration & $\overline{\text { LSJ }}$ & Expt. & $\overline{\text { FAC }}$ & CIV3 & OTC99 & Chianti \\
\hline 1 & $2 s^{2} 2 p^{2}$ & ${ }^{3} P_{0}$ & & 0.0000 & 0.0000 & & 0.000 \\
\hline 2 & $2 s^{2} 2 p^{2}$ & ${ }^{3} P_{1}$ & 0.0232 & 0.0226 & 0.0226 & & 0.023 \\
\hline 3 & $2 s^{2} 2 p^{2}$ & ${ }^{3} P_{2}$ & 0.0585 & 0.0570 & 0.0575 & & 0.058 \\
\hline 4 & $2 s^{2} 2 p^{2}$ & ${ }^{1} D_{2}$ & 0.4823 & 0.5081 & 0.5110 & & 0.506 \\
\hline 5 & $2 s^{2} 2 p^{2}$ & ${ }^{1} S_{0}$ & 0.9823 & 0.9949 & 1.0500 & & 0.948 \\
\hline 6 & $2 s 2 p^{3}$ & ${ }^{5} S_{2}$ & 1.3690 & 1.3025 & 1.3416 & & 1.261 \\
\hline 7 & $2 s 2 p^{3}$ & ${ }^{3} D_{3}$ & 2.6631 & 2.6893 & 2.6867 & 2.6621 & 2.673 \\
\hline 8 & $2 s 2 p^{3}$ & ${ }^{3} D_{2}$ & 2.6636 & 2.6898 & 2.6871 & 2.6627 & 2.670 \\
\hline 9 & $2 s 2 p^{3}$ & ${ }^{3} D_{1}$ & 2.6649 & 2.6909 & 2.6881 & 2.6602 & 2.671 \\
\hline 10 & $2 s 2 p^{3}$ & ${ }^{3} P_{2}$ & 3.1359 & 3.1668 & 3.1809 & 3.1354 & 3.136 \\
\hline 11 & $2 s 2 p^{3}$ & ${ }^{3} P_{1}$ & 3.1349 & 3.1668 & 3.1817 & 3.1350 & 3.137 \\
\hline 12 & $2 s 2 p^{3}$ & ${ }^{3} P_{0}$ & 3.1354 & 3.1678 & 3.1805 & 3.1351 & 3.139 \\
\hline 13 & $2 s 2 p^{3}$ & ${ }^{1} D_{2}$ & 4.0132 & 4.1204 & 4.0740 & 4.0133 & 4.144 \\
\hline 14 & $2 s 2 p^{3}$ & ${ }^{3} S_{1}$ & 4.0728 & 4.1651 & 4.1190 & 4.0720 & 4.205 \\
\hline 15 & $2 s 2 p^{3}$ & ${ }^{1} P_{1}$ & 4.4903 & 4.6007 & 4.5760 & 4.4911 & 4.610 \\
\hline 16 & $2 p^{4}$ & ${ }^{3} P_{2}$ & 6.1488 & 6.2336 & 6.2545 & 6.1479 & 6.262 \\
\hline 17 & $2 p^{4}$ & ${ }^{3} P_{1}$ & 6.1903 & 6.2752 & 6.2950 & 6.1896 & 6.303 \\
\hline 18 & $2 p^{4}$ & ${ }^{3} P_{0}$ & 6.2065 & 6.2887 & 6.3111 & 6.2047 & 6.320 \\
\hline 19 & $2 p^{4}$ & ${ }^{1} D_{2}$ & 6.5566 & 6.6892 & 6.6769 & 6.5570 & 6.745 \\
\hline 20 & $2 p^{4}$ & ${ }^{1} S_{0}$ & 7.4696 & 7.6367 & 7.6551 & 7.4702 & 7.685 \\
\hline 21 & $2 s^{2} 2 p 3 s$ & ${ }^{3} P_{0}$ & & 14.7296 & 14.8425 & & 14.991 \\
\hline 22 & $2 s^{2} 2 p 3 s$ & ${ }^{3} P_{1}$ & 14.7933 & 14.7453 & 14.8574 & 14.7925 & 15.006 \\
\hline 23 & $2 s^{2} 2 p 3 s$ & ${ }^{3} P_{2}$ & 14.8400 & 14.7911 & 14.9004 & 14.8391 & 15.051 \\
\hline 24 & $2 s^{2} 2 p 3 s$ & ${ }^{1} P_{1}$ & 14.9525 & 14.9307 & 15.0370 & 14.9521 & 15.170 \\
\hline 25 & $2 s^{2} 2 p 3 p$ & ${ }^{1} P_{1}$ & & 15.4136 & 15.4789 & & 15.662 \\
\hline 26 & $2 s^{2} 2 p 3 p$ & ${ }^{3} D_{1}$ & & 15.4788 & 15.5482 & & 15.715 \\
\hline 27 & $2 s^{2} 2 p 3 p$ & ${ }^{3} D_{2}$ & & 15.4937 & 15.5636 & & 15.727 \\
\hline 28 & $2 s^{2} 2 p 3 p$ & ${ }^{3} D_{3}$ & & 15.5333 & 15.6014 & & 15.767 \\
\hline 29 & $2 s^{2} 2 p 3 p$ & ${ }^{3} S_{1}$ & & 15.6022 & 15.6734 & & 15.838 \\
\hline 30 & $2 s^{2} 2 p 3 p$ & ${ }^{3} P_{0}$ & & 15.6550 & 15.7213 & & 15.964 \\
\hline 31 & $2 s^{2} 2 p 3 p$ & ${ }^{3} P_{1}$ & & 15.6751 & 15.7405 & & 15.982 \\
\hline 32 & $2 s^{2} 2 p 3 p$ & ${ }^{3} P_{2}$ & & 15.6944 & 15.7586 & & 16.003 \\
\hline 33 & $2 s^{2} 2 p 3 p$ & ${ }^{1} D_{2}$ & & 15.9415 & 15.9934 & & 16.158 \\
\hline 34 & $2 s 2 p^{2}\left({ }^{2} S\right) 3 s$ & ${ }^{3} S_{1}$ & & 16.1831 & & 16.2622 & \\
\hline 35 & $2 s 2 p^{2}\left({ }^{2} D\right) 3 s$ & ${ }^{1} D_{2}$ & & 16.2017 & & 16.2818 & \\
\hline 36 & $2 s^{2} 2 p 3 p$ & ${ }^{1} S_{0}$ & & 16.2121 & 16.2563 & & 16.407 \\
\hline 37 & $2 s 2 p^{2}\left({ }^{4} P\right) 3 s$ & ${ }^{5} P_{3}$ & & 16.2309 & & 16.3109 & \\
\hline 38 & $2 s^{2} 2 p 3 d$ & ${ }^{3} F_{2}$ & & 16.2850 & 16.3183 & 16.3057 & 16.500 \\
\hline 39 & $2 s^{2} 2 p 3 d$ & ${ }^{3} F_{3}$ & & 16.3207 & 16.3505 & & 16.529 \\
\hline 40 & $2 s^{2} 2 p 3 d$ & ${ }^{1} D_{2}$ & 16.3486 & 16.3352 & 16.3571 & 16.3484 & 16.551 \\
\hline 41 & $2 s^{2} 2 p 3 d$ & ${ }^{3} F_{4}$ & & 16.3492 & 16.3807 & & 16.561 \\
\hline 42 & $2 s^{2} 2 p 3 d$ & ${ }^{3} D_{1}$ & 16.4772 & 16.4629 & 16.5016 & 16.4773 & 16.692 \\
\hline 43 & $2 s^{2} 2 p 3 d$ & ${ }^{3} D_{2}$ & 16.4852 & 16.4748 & 16.5075 & 16.4848 & 16.698 \\
\hline 44 & $2 s^{2} 2 p 3 d$ & ${ }^{3} D_{3}$ & 16.5070 & 16.4939 & 16.5282 & 16.5071 & 16.720 \\
\hline 45 & $2 s^{2} 2 p 3 d$ & ${ }^{3} P_{2}$ & 16.5455 & 16.5290 & 16.5595 & 16.5455 & 16.752 \\
\hline 46 & $2 s^{2} 2 p 3 d$ & ${ }^{3} P_{1}$ & 16.5569 & 16.5404 & 16.5692 & 16.5566 & 16.761 \\
\hline 47 & $2 s^{2} 2 p 3 d$ & ${ }^{3} P_{0}$ & 16.5635 & 16.5470 & 16.5746 & 16.5630 & 16.766 \\
\hline 48 & $2 s 2 p^{2}\left({ }^{2} S\right) 3 s$ & ${ }^{1} S_{0}$ & & 16.6427 & & & \\
\hline 49 & $2 s 2 p^{2}\left({ }^{2} D\right) 3 s$ & ${ }^{3} D_{1}$ & & 16.6575 & & & \\
\hline 50 & $2 s 2 p^{2}\left({ }^{4} P\right) 3 s$ & ${ }^{3} P_{2}$ & & 16.6888 & & & \\
\hline 51 & $2 s^{2} 2 p 3 d$ & ${ }^{1} P_{1}$ & 16.7542 & 16.7481 & 16.7985 & 16.7529 & 16.987 \\
\hline 52 & $2 s^{2} 2 p 3 d$ & ${ }^{1} F_{3}$ & 16.7471 & 16.7602 & 16.8024 & 16.7468 & 16.998 \\
\hline 53 & $2 s 2 p^{2}\left({ }^{4} P\right) 3 p$ & ${ }^{3} S_{1}$ & 16.9363 & 16.8788 & & 16.9364 & \\
\hline 54 & $2 s 2 p^{2}\left({ }^{2} S\right) 3 p$ & ${ }^{3} P_{0}$ & & 16.9209 & & & \\
\hline 55 & $2 s 2 p^{2}\left({ }^{2} S\right) 3 p$ & ${ }^{1} P_{1}$ & & 16.9263 & & & \\
\hline 56 & $2 s 2 p^{2}\left({ }^{2} D\right) 3 p$ & ${ }^{1} D_{2}$ & & 16.9374 & & & \\
\hline 57 & $2 s 2 p^{2}\left({ }^{2} D\right) 3 p$ & ${ }^{1} F_{3}$ & & 16.9550 & & & \\
\hline 58 & $2 s 2 p^{2}\left({ }^{4} P\right) 3 p$ & ${ }^{5} D_{4}$ & & 16.9804 & & & \\
\hline 59 & $2 s 2 p^{2}\left({ }^{2} D\right) 3 p$ & ${ }^{1} P_{1}$ & & 17.0150 & & & \\
\hline
\end{tabular}


Table I-continued ...

\begin{tabular}{|c|c|c|c|c|c|c|c|}
\hline Index & Configuration & LSJ & Expt. & $\overline{\mathrm{FAC}}$ & CIV3 & OTC99 & Chianti \\
\hline 60 & $2 s 2 p^{2}\left({ }^{4} P\right) 3 p$ & ${ }^{5} P_{2}$ & & 17.0251 & & & \\
\hline 61 & $2 s 2 p^{2}\left({ }^{4} P\right) 3 p$ & ${ }^{5} D_{3}$ & & 17.0429 & & & \\
\hline 62 & $2 s 2 p^{2}\left({ }^{2} S\right) 3 p$ & ${ }^{5} P_{1}$ & & 17.2390 & & & \\
\hline 63 & $2 s 2 p^{2}\left({ }^{4} P\right) 3 p$ & ${ }^{3} D_{2}$ & 17.2788 & 17.2536 & & 17.2784 & \\
\hline 64 & $2 s 2 p^{2}\left({ }^{4} P\right) 3 p$ & ${ }^{3} D_{3}$ & 17.3049 & 17.2786 & & 17.3050 & \\
\hline 65 & $2 s 2 p^{2}\left({ }^{4} P\right) 3 p$ & ${ }^{5} P_{2}$ & & 17.3325 & & & \\
\hline 66 & $2 s 2 p^{2}\left({ }^{2} P\right) 3 p$ & ${ }^{3} P_{0}$ & & 17.3858 & & & \\
\hline & availab & on re & & & & & \\
\hline 557 & $2 s 2 p^{2}\left({ }^{4} P\right) 4 f$ & ${ }^{3} G_{4}$ & & 24.4150 & & & \\
\hline 558 & $2 s 2 p^{2}\left({ }^{4} P\right) 4 f$ & ${ }^{3} G_{3}$ & & 24.4166 & & & \\
\hline 559 & $2 s 2 p^{2}\left({ }^{4} P\right) 4 f$ & ${ }^{5} D_{4}$ & & 24.4195 & & & \\
\hline 560 & $2 s 2 p^{2}\left({ }^{4} P\right) 4 f$ & ${ }^{3} G_{3}$ & & 24.4264 & & & \\
\hline
\end{tabular}


TABLE II:

\begin{tabular}{|c|c|c|c|c|c|c|}
\hline Index & Configuration & LSJ & Expt. & $\overline{\mathrm{FAC}}$ & CLT00 & Chianti \\
\hline 1 & $2 s^{2} 2 p$ & ${ }^{2} P_{1 / 2}$ & & 0.0000 & & 0.0000 \\
\hline 2 & $2 s^{2} 2 p$ & ${ }^{2} P_{3 / 2}$ & 0.0637 & 0.0623 & 0.0636 & 0.0637 \\
\hline 3 & $2 s 2 p^{2}$ & ${ }^{4} P_{1 / 2}$ & 1.4672 & 1.4335 & 1.4756 & 1.4672 \\
\hline 4 & $2 s 2 p^{2}$ & ${ }^{4} P_{3 / 2}$ & 1.4898 & 1.4567 & 1.4977 & 1.4898 \\
\hline 5 & $2 s 2 p^{2}$ & ${ }^{4} P_{5 / 2}$ & 1.5224 & 1.4880 & 1.5304 & 1.5224 \\
\hline 6 & $2 s 2 p^{2}$ & ${ }^{2} D_{5 / 2}$ & 2.6231 & 2.6622 & 2.6230 & 2.6231 \\
\hline 7 & $2 s 2 p^{2}$ & ${ }^{2} D_{3 / 2}$ & 2.6234 & 2.6620 & 2.6231 & 2.6234 \\
\hline 8 & $2 s 2 p^{2}$ & ${ }^{2} S_{1 / 2}$ & 3.3505 & 3.4029 & 3.3503 & 3.3505 \\
\hline 9 & $2 s 2 p^{2}$ & ${ }^{2} P_{1 / 2}$ & 3.5543 & 3.6325 & 3.5545 & 3.5543 \\
\hline 10 & $2 s 2 p^{2}$ & ${ }^{2} P_{3 / 2}$ & 3.5907 & 3.6687 & 3.5909 & 3.5907 \\
\hline 11 & $2 p^{3}$ & ${ }^{4} S_{3 / 2}$ & 4.6414 & 4.6274 & 4.6488 & 4.6414 \\
\hline 12 & $2 p^{3}$ & ${ }^{2} D_{3 / 2}$ & 5.2437 & 5.2973 & 5.2438 & 5.2437 \\
\hline 13 & $2 p^{3}$ & ${ }^{2} D_{5 / 2}$ & 5.2439 & 5.2984 & 5.2442 & 5.2439 \\
\hline 14 & $2 p^{3}$ & ${ }^{2} P_{1 / 2}$ & 5.8937 & 5.9806 & 5.8937 & 5.8937 \\
\hline 15 & $2 p^{3}$ & ${ }^{2} P_{3 / 2}$ & 5.8994 & 5.9832 & 5.8996 & 5.8995 \\
\hline 16 & $2 s^{2} 3 s$ & ${ }^{2} S_{1 / 2}$ & & 16.5368 & 16.6033 & 16.4970 \\
\hline 17 & $2 s^{2} 3 p$ & ${ }^{2} P_{1 / 2}$ & & 17.2881 & 17.6598 & 17.2366 \\
\hline 18 & $2 s^{2} 3 p$ & ${ }^{2} P_{3 / 2}$ & & 17.3046 & 17.6792 & 17.2548 \\
\hline 19 & $2 s^{2} 3 d$ & ${ }^{2} D_{3 / 2}$ & 18.0363 & 17.9972 & 18.0364 & 17.9443 \\
\hline 20 & $2 s^{2} 3 d$ & ${ }^{2} D_{5 / 2}$ & 18.0406 & 18.0014 & 18.0406 & 17.9493 \\
\hline 21 & $2 s 2 p\left({ }^{3} P\right) 3 s$ & ${ }^{4} P_{1 / 2}$ & 18.1600 & 18.1088 & 18.1683 & 18.0608 \\
\hline 22 & $2 s 2 p\left({ }^{3} P\right) 3 s$ & ${ }^{4} P_{3 / 2}$ & 18.1810 & 18.1293 & 18.1890 & 18.0818 \\
\hline 23 & $2 s 2 p\left({ }^{3} P\right) 3 s$ & ${ }^{4} P_{5 / 2}$ & 18.2215 & 18.1675 & 18.2296 & 18.1209 \\
\hline 24 & $2 s 2 p\left({ }^{3} P\right) 3 s$ & ${ }^{2} P_{1 / 2}$ & 18.5084 & 18.5012 & 18.5086 & 18.4184 \\
\hline 25 & $2 s 2 p\left({ }^{3} P\right) 3 s$ & ${ }^{2} P_{3 / 2}$ & 18.5521 & 18.5431 & 18.5518 & 18.4615 \\
\hline 26 & $2 s 2 p\left({ }^{3} P\right) 3 p$ & ${ }^{4} D_{1 / 2}$ & & 18.7772 & & 18.7209 \\
\hline 27 & $2 s 2 p\left({ }^{3} P\right) 3 p$ & ${ }^{4} D_{3 / 2}$ & & 18.7954 & & 18.7405 \\
\hline 28 & $2 s 2 p\left({ }^{3} P\right) 3 p$ & ${ }^{2} P_{1 / 2}$ & 18.8139 & 18.8146 & 18.8140 & 18.7593 \\
\hline 29 & $2 s 2 p\left({ }^{3} P\right) 3 p$ & ${ }^{2} P_{3 / 2}$ & 18.8336 & 18.8238 & 18.8442 & 18.7683 \\
\hline 30 & $2 s 2 p\left({ }^{3} P\right) 3 p$ & ${ }^{4} D_{5 / 2}$ & & 18.8269 & & 18.7769 \\
\hline 31 & $2 s 2 p\left({ }^{3} P\right) 3 p$ & ${ }^{4} D_{7 / 2}$ & & 18.8607 & & 18.8121 \\
\hline 32 & $2 s 2 p\left({ }^{3} P\right) 3 p$ & ${ }^{4} S_{3 / 2}$ & & 18.9586 & & 18.9056 \\
\hline 33 & $2 s 2 p\left({ }^{3} P\right) 3 p$ & ${ }^{4} P_{1 / 2}$ & & 19.0822 & & 18.9858 \\
\hline 34 & $2 s 2 p\left({ }^{3} P\right) 3 p$ & ${ }^{4} P_{3 / 2}$ & & 19.0993 & & 19.0046 \\
\hline 35 & $2 s 2 p\left({ }^{3} P\right) 3 p$ & ${ }^{4} P_{5 / 2}$ & & 19.1179 & & 19.0227 \\
\hline 36 & $2 s 2 p\left({ }^{3} P\right) 3 p$ & ${ }^{2} D_{3 / 2}$ & 19.1890 & 19.1919 & 19.1892 & 19.1028 \\
\hline 37 & $2 s 2 p\left({ }^{3} P\right) 3 p$ & ${ }^{2} D_{5 / 2}$ & 19.2301 & 19.2327 & 19.2302 & 19.1437 \\
\hline 38 & $2 s 2 p\left({ }^{3} P\right) 3 d$ & ${ }^{4} F_{3 / 2}$ & & 19.3973 & & 19.3414 \\
\hline 39 & $2 s 2 p\left({ }^{3} P\right) 3 d$ & ${ }^{4} F_{5 / 2}$ & & 19.4121 & & 19.3544 \\
\hline 40 & $2 s 2 p\left({ }^{3} P\right) 3 d$ & ${ }^{4} F_{7 / 2}$ & & 19.4318 & & 19.3738 \\
\hline 41 & $2 s 2 p\left({ }^{3} P\right) 3 p$ & ${ }^{2} S_{1 / 2}$ & 19.4337 & 19.4463 & 19.4335 & 19.3548 \\
\hline 42 & $2 s 2 p\left({ }^{3} P\right) 3 d$ & ${ }^{4} F_{9 / 2}$ & & 19.4548 & & 19.4014 \\
\hline 43 & $2 s 2 p\left({ }^{3} P\right) 3 d$ & ${ }^{4} D_{1 / 2}$ & 19.6004 & 19.5722 & & 19.5081 \\
\hline 44 & $2 s 2 p\left({ }^{3} P\right) 3 d$ & ${ }^{4} D_{3 / 2}$ & & 19.5757 & 19.6090 & 19.5096 \\
\hline 45 & $2 s 2 p\left({ }^{3} P\right) 3 d$ & ${ }^{4} D_{5 / 2}$ & 19.6046 & 19.5820 & 19.6138 & 19.5130 \\
\hline 46 & $2 s 2 p\left({ }^{3} P\right) 3 d$ & ${ }^{4} D_{7 / 2}$ & 19.6271 & 19.5997 & 19.6353 & 19.5349 \\
\hline 47 & $2 s 2 p\left({ }^{3} P\right) 3 d$ & ${ }^{2} D_{3 / 2}$ & 19.6260 & 19.6198 & 19.6260 & 19.5313 \\
\hline 48 & $2 s 2 p\left({ }^{3} P\right) 3 d$ & ${ }^{2} D_{5 / 2}$ & 19.6331 & 19.6240 & 19.6332 & 19.5390 \\
\hline 49 & $2 s 2 p\left({ }^{3} P\right) 3 d$ & ${ }^{4} P_{5 / 2}$ & 19.6917 & 19.6568 & 19.6999 & 19.5923 \\
\hline \multicolumn{7}{|c|}{ available on request } \\
\hline 298 & $2 s 2 p\left({ }^{3} P\right) 5 p$ & ${ }^{2} D_{3 / 2}$ & & 28.3518 & & \\
\hline 299 & $2 s 2 p\left({ }^{3} P\right) 5 p$ & ${ }^{2} D_{5 / 2}$ & & 28.3536 & & \\
\hline 300 & $2 s 2 p\left({ }^{3} P\right) 5 p$ & ${ }^{2} P_{1 / 2}$ & & 28.3581 & & \\
\hline 301 & $2 s 2 p\left({ }^{3} P\right) 5 p$ & ${ }^{4} S_{3 / 2}$ & & 28.3606 & & \\
\hline 302 & $2 s 2 p\left({ }^{3} P\right) 5 p$ & ${ }^{4} P_{1 / 2}$ & & 28.3967 & & \\
\hline 303 & $2 s 2 p\left({ }^{3} P\right) 5 d$ & ${ }^{2} F_{7 / 2}$ & & 28.4662 & 28.3994 & \\
\hline 304 & $2 s 2 p\left({ }^{3} P\right) 5 d$ & ${ }^{2} F_{5 / 2}$ & & 28.4663 & 28.4308 & \\
\hline 305 & $2 s 2 p\left({ }^{3} P\right) 5 d$ & ${ }^{2} D_{3 / 2}$ & & 28.4665 & 28.3992 & \\
\hline 306 & $2 s 2 p\left({ }^{3} P\right) 5 d$ & ${ }^{4} P_{5 / 2}$ & & 28.4677 & & \\
\hline
\end{tabular}


Table II-continued ...

\begin{tabular}{|c|c|c|c|c|c|c|}
\hline Index & Configuration & $\overline{\overline{\text { LSJ }}}$ & $\overline{\text { Expt }}$ & $\overline{\mathrm{FAC}}$ & CLT00 & Chianti \\
\hline 307 & $2 s 2 p\left({ }^{3} P\right) 5 d$ & ${ }^{2} P_{1 / 2}$ & & 28.4976 & & \\
\hline 308 & $2 s 2 p\left({ }^{3} P\right) 5 d$ & ${ }^{4} P_{3 / 2}$ & & 28.4983 & & \\
\hline 309 & $2 s 2 p\left({ }^{3} P\right) 5 g$ & ${ }^{2} G_{7 / 2}$ & & 28.5209 & & \\
\hline 310 & $2 s 2 p\left({ }^{3} P\right) 5 g$ & ${ }^{4} F_{9 / 2}$ & & 28.5211 & & \\
\hline 311 & $2 s 2 p\left({ }^{3} P\right) 5 f$ & ${ }^{2} F_{5 / 2}$ & & 28.5242 & & \\
\hline 312 & $2 s 2 p\left({ }^{3} P\right) 5 f$ & ${ }^{4} D_{7 / 2}$ & & 28.5246 & & \\
\hline 313 & $2 s 2 p\left({ }^{3} P\right) 5 g$ & ${ }^{2} H_{9 / 2}$ & & 28.5328 & & \\
\hline 314 & $2 s 2 p\left({ }^{3} P\right) 5 g$ & ${ }^{2} H_{11 / 2}$ & & 28.5330 & & \\
\hline 315 & $2 s 2 p\left({ }^{3} P\right) 5 f$ & ${ }^{4} D_{5 / 2}$ & & 28.5371 & & \\
\hline 316 & $2 s 2 p\left({ }^{3} P\right) 5 g$ & ${ }^{4} F_{7 / 2}$ & & 28.5373 & & \\
\hline 317 & $2 s 2 p\left({ }^{3} P\right) 5 f$ & ${ }^{2} G_{7 / 2}$ & & 28.5443 & & \\
\hline 318 & $2 s 2 p\left({ }^{3} P\right) 5 f$ & ${ }^{2} G_{9 / 2}$ & & 28.5446 & & \\
\hline 319 & $2 s 2 p\left({ }^{3} P\right) 5 f$ & ${ }^{2} D_{3 / 2}$ & & 28.5578 & & \\
\hline 320 & $2 s 2 p\left({ }^{3} P\right) 5 f$ & ${ }^{2} D_{5 / 2}$ & & 28.5580 & & \\
\hline
\end{tabular}


TABLE III:

\begin{tabular}{|c|c|c|c|c|c|c|}
\hline Index & Configuration & $\overline{\mathrm{LSJ}}$ & Expt. & $\overline{\overline{\text { FAC }}}$ & "CT01 & Chianti \\
\hline 1 & $2 s^{2}$ & ${ }^{1} S_{0}$ & & 0.000000 & 0.000000 & 0.000000 \\
\hline 2 & $2 s 2 p$ & ${ }^{3} P_{0}$ & 1.547354 & 1.544777 & 1.547362 & 1.556960 \\
\hline 3 & $2 s 2 p$ & ${ }^{3} P_{1}$ & 1.568696 & 1.565661 & 1.568859 & 1.578420 \\
\hline 4 & $2 s 2 p$ & ${ }^{3} P_{2}$ & 1.615845 & 1.611971 & 1.615744 & 1.625680 \\
\hline 5 & $2 s 2 p$ & ${ }^{1} P_{1}$ & 3.004365 & 3.050184 & 3.004353 & 3.131050 \\
\hline 6 & $2 p^{2}$ & ${ }^{3} P_{0}$ & 4.043030 & 4.031292 & 4.043251 & 4.069800 \\
\hline 7 & $2 p^{2}$ & ${ }^{3} P_{1}$ & 4.068764 & 4.060359 & 4.068830 & 4.095150 \\
\hline 8 & $2 p^{2}$ & ${ }^{3} P_{2}$ & 4.109507 & 4.099710 & 4.109646 & 4.136090 \\
\hline 9 & $2 p^{2}$ & ${ }^{1} D_{2}$ & 4.512617 & 4.540432 & 4.512730 & 4.614360 \\
\hline 10 & $2 p^{2}$ & ${ }^{1} S_{0}$ & 5.547427 & 5.624651 & 5.547728 & 5.689970 \\
\hline 11 & $2 s 3 s$ & ${ }^{3} S_{1}$ & 20.145382 & 20.113743 & 20.146660 & 19.776400 \\
\hline 12 & $2 s 3 s$ & ${ }^{1} S_{0}$ & 20.428877 & 20.417677 & 20.429729 & 20.148800 \\
\hline 13 & $2 s 3 p$ & ${ }^{1} P_{1}$ & 20.822817 & 20.825602 & 20.826143 & 20.658401 \\
\hline 14 & $2 s 3 p$ & ${ }^{3} P_{0}$ & 20.824370 & 20.850800 & 20.850365 & 20.677999 \\
\hline 15 & $2 s 3 p$ & ${ }^{3} P_{1}$ & 20.824370 & 20.858654 & 20.860752 & 20.677999 \\
\hline 16 & $2 s 3 p$ & ${ }^{3} P_{2}$ & 20.824370 & 20.868895 & 20.866348 & 20.697599 \\
\hline 17 & $2 s 3 d$ & ${ }^{3} D_{1}$ & & 21.243521 & 21.244827 & 21.207199 \\
\hline 18 & $2 s 3 d$ & ${ }^{3} D_{2}$ & 21.251204 & 21.245701 & 21.253338 & 21.226801 \\
\hline 19 & $2 s 3 d$ & ${ }^{3} D_{3}$ & 21.255487 & 21.249430 & 21.254696 & 21.226801 \\
\hline 20 & $2 s 3 d$ & ${ }^{1} D_{2}$ & 21.517658 & 21.541889 & 21.515211 & 21.599201 \\
\hline 21 & $2 p 3 s$ & ${ }^{3} P_{0}$ & & 22.100626 & 22.088411 & 22.030401 \\
\hline 22 & $2 p 3 s$ & ${ }^{3} P_{1}$ & & 22.120941 & 22.114548 & 22.069599 \\
\hline 23 & $2 p 3 s$ & ${ }^{3} P_{2}$ & 22.145796 & 22.170929 & 22.158224 & 22.128399 \\
\hline 24 & $2 p 3 s$ & ${ }^{1} P_{1}$ & 22.515768 & 22.456436 & 22.516539 & 22.481199 \\
\hline 25 & $2 p 3 p$ & ${ }^{1} S_{0}$ & & 22.543678 & 22.533634 & 22.598801 \\
\hline 26 & $2 p 3 p$ & ${ }^{1} P_{1}$ & & 22.622646 & 22.600367 & 22.696800 \\
\hline 27 & $2 p 3 p$ & ${ }^{1} D_{2}$ & & 22.639996 & 22.619520 & 22.716400 \\
\hline 28 & $2 p 3 p$ & ${ }^{3} D_{3}$ & 22.666128 & 22.687033 & 22.666525 & 22.794800 \\
\hline 29 & $2 p 3 p$ & ${ }^{3} S_{1}$ & 22.785503 & 22.804148 & 22.785364 & 22.931999 \\
\hline 30 & $2 p 3 p$ & ${ }^{3} P_{0}$ & 22.847015 & 22.902258 & 22.846100 & 23.010399 \\
\hline 31 & $2 p 3 p$ & ${ }^{3} P_{1}$ & 22.873987 & 22.922987 & 22.874140 & 23.049601 \\
\hline 32 & $2 p 3 p$ & ${ }^{3} P_{2}$ & 22.894491 & 22.946426 & 22.895081 & 23.069201 \\
\hline 33 & $2 p 3 d$ & ${ }^{3} F_{2}$ & & 22.973572 & 22.944773 & 23.147600 \\
\hline 34 & $2 p 3 d$ & ${ }^{1} F_{3}$ & & 23.014633 & 22.981279 & 23.206400 \\
\hline \multicolumn{7}{|c|}{ available on request } \\
\hline 323 & $2 p 7 h$ & ${ }^{3} I_{5}$ & & 34.357094 & & \\
\hline 324 & $2 p 7 i$ & ${ }^{3} H_{5}$ & & 34.357132 & & \\
\hline 325 & $2 p 7 i$ & ${ }^{3} J_{6}$ & & 34.357189 & & \\
\hline 326 & $2 p 7 g$ & ${ }^{3} G_{4}$ & & 34.357510 & & \\
\hline 327 & $2 p 7 g$ & ${ }^{3} H_{5}$ & & 34.357689 & & \\
\hline 328 & $2 p 7 i$ & ${ }^{3} I_{7}$ & & 34.357918 & & \\
\hline 329 & $2 p 7 i$ & ${ }^{3} J_{8}$ & & 34.357975 & & \\
\hline 330 & $2 p 7 h$ & ${ }^{3} H_{6}$ & & 34.358189 & & \\
\hline 331 & $2 p 7 h$ & ${ }^{3} I_{7}$ & & 34.358253 & & \\
\hline 332 & $2 p 7 i$ & ${ }^{3} H_{4}$ & & 34.358383 & & \\
\hline 333 & $2 p 7 i$ & ${ }^{3} J_{5}$ & & 34.358437 & & \\
\hline 334 & $2 p 7 f$ & ${ }^{3} G_{5}$ & & 34.358879 & & \\
\hline 335 & $2 p 7 f$ & ${ }^{3} G_{3}$ & & 34.359028 & & \\
\hline 336 & $2 p 7 h$ & ${ }^{3} G_{3}$ & & 34.359108 & & \\
\hline 337 & $2 p 7 h$ & ${ }^{3} H_{4}$ & & 34.359184 & & \\
\hline 338 & $2 p 7 g$ & ${ }^{3} F_{3}$ & & 34.359249 & & \\
\hline 339 & $2 p 7 g$ & ${ }^{3} G_{4}$ & & 34.359264 & & \\
\hline 340 & $2 p 7 f$ & ${ }^{3} D_{2}$ & & 34.360397 & & \\
\hline 341 & $2 p 7 g$ & ${ }^{3} H_{6}$ & & 34.360783 & & \\
\hline 342 & $2 p 7 g$ & ${ }^{3} G_{5}$ & & 34.360950 & & \\
\hline 343 & $2 p 7 g$ & ${ }^{3} F_{2}$ & & 34.362793 & & \\
\hline 344 & $2 p 7 g$ & ${ }^{3} \mathrm{H}_{3}$ & & 34.362942 & & \\
\hline 345 & $2 p 7 f$ & ${ }^{3} G_{4}$ & & 34.363071 & & \\
\hline 346 & $2 p 7 f$ & ${ }^{3} D_{1}$ & & 34.364277 & & \\
\hline
\end{tabular}


Table III-continued ...

\begin{tabular}{|c|c|c|c|c|c|c|}
\hline Index & Configuration & LSJ & Expt. & $\overline{\mathrm{FAC}}$ & CT01 & Chianti \\
\hline 347 & $2 p 7 d$ & ${ }^{3} F_{3}$ & & 34.364918 & & \\
\hline 348 & $2 p 7 p$ & ${ }^{3} P_{0}$ & & 34.366123 & & \\
\hline 349 & $2 p 7 f$ & ${ }^{3} F_{2}$ & & 34.367489 & & \\
\hline 350 & $2 p 7 d$ & ${ }^{3} D_{1}$ & & 34.367958 & & \\
\hline
\end{tabular}


TABLE VII:

\begin{tabular}{|c|c|c|c|c|c|c|c|c|c|c|c|}
\hline \multirow[t]{2}{*}{ i } & \multirow[t]{2}{*}{$\mathrm{j}$} & \multicolumn{10}{|c|}{$\begin{array}{l}\text { Collision strength } \Omega \\
\text { cattered electron energy }\end{array}$} \\
\hline & & 10 & 50 & 100 & 200 & 400 & 600 & 800 & 1000 & 1500 & 2000 \\
\hline 1 & 2 & $9.040-02$ & $7.805-02$ & $6.377-02$ & $4.670-02$ & $2.733-02$ & $1.793-02$ & $1.274-02$ & $9.500-03$ & $5.314-03$ & $3.353-03$ \\
\hline 1 & 3 & $4.495-01$ & $2.123-01$ & $9.070-02$ & $7.488-02$ & $6.186-02$ & $3.720-02$ & $3.540-02$ & $3.593-02$ & $3.053-02$ & $2.962-02$ \\
\hline 1 & 4 & 3.933-02 & $3.752-02$ & $3.260-02$ & $2.324-02$ & $1.267-02$ & $8.160-03$ & $5.565-03$ & $3.977-03$ & $2.164-03$ & $1.345-03$ \\
\hline 1 & 5 & $5.625-03$ & $5.129-03$ & $4.243-03$ & $2.870-03$ & $1.423-03$ & $8.418-04$ & $5.267-04$ & $3.455-04$ & $1.633-04$ & 8.897-05 \\
\hline 1 & 6 & $1.274-02$ & $1.134-02$ & $9.723-03$ & $7.414-03$ & $4.620-03$ & $3.097-03$ & $2.234-03$ & $1.683-03$ & $9.516-04$ & $6.050-04$ \\
\hline 1 & 7 & $4.332-05$ & $3.274-05$ & $2.340-05$ & $3.693-05$ & $8.214-05$ & $3.605-05$ & $4.109-05$ & $4.763-05$ & $3.705-05$ & $3.546-05$ \\
\hline 1 & 8 & $1.060-02$ & $9.376-03$ & 7.984-03 & 6.061-03 & $3.767-03$ & $2.522-03$ & $1.819-03$ & $1.372-03$ & $7.751-04$ & $4.927-04$ \\
\hline 1 & 9 & $3.492-01$ & $3.815-01$ & $4.157-01$ & $4.652-01$ & $5.344-01$ & $5.842-01$ & $6.222-01$ & $6.528-01$ & $7.101-01$ & $7.532-01$ \\
\hline 1 & 10 & $2.621-03$ & $2.290-03$ & $1.928-03$ & $1.455-03$ & 8.993-04 & $5.972-04$ & $4.291-04$ & $3.226-04$ & $1.805-04$ & $1.138-04$ \\
\hline 1 & 11 & $2.566-01$ & $2.860-01$ & $3.147-01$ & $3.520-01$ & $4.045-01$ & $4.433-01$ & $4.730-01$ & $4.970-01$ & $5.422-01$ & $5.762-01$ \\
\hline 1 & 12 & $1.576-03$ & $1.378-03$ & $1.160-03$ & $8.740-04$ & $5.385-04$ & $3.568-04$ & $2.559-04$ & $1.921-04$ & $1.072-04$ & $6.740-05$ \\
\hline 1 & 13 & $6.334-03$ & $5.508-03$ & $4.635-03$ & $3.525-03$ & $2.203-03$ & $1.475-03$ & $1.067-03$ & 8.064-04 & $4.557-04$ & $2.897-04$ \\
\hline 1 & 14 & 2.139-01 & $2.378-01$ & $2.612-01$ & $2.912-01$ & $3.347-01$ & $3.673-01$ & 3.931-01 & 4.141-01 & $4.535-01$ & $4.838-01$ \\
\hline 1 & 15 & $2.367-03$ & $2.026-03$ & $1.685-03$ & $1.278-03$ & $8.017-04$ & $5.371-04$ & $3.925-04$ & $3.011-04$ & $1.774-04$ & $1.202-04$ \\
\hline 1 & 16 & $1.808-03$ & $1.865-03$ & $1.961-03$ & $2.120-03$ & $2.848-03$ & $3.401-03$ & 2.923-03 & $2.600-03$ & $2.827-03$ & 2.903-03 \\
\hline 1 & 17 & $9.217-05$ & 8.519-05 & $7.205-05$ & $4.936-05$ & $2.521-05$ & $1.520-05$ & $9.707-06$ & $6.486-06$ & $3.087-06$ & $1.687-06$ \\
\hline 1 & 18 & $7.107-04$ & $6.728-04$ & $6.340-04$ & 5.944-04 & $5.446-04$ & $5.185-04$ & $5.047-04$ & $4.957-04$ & $4.824-04$ & $4.762-04$ \\
\hline 1 & 19 & $3.261-04$ & $2.968-04$ & $2.487-04$ & $1.704-04$ & $8.758-05$ & $5.275-05$ & $3.378-05$ & $2.267-05$ & $1.080-05$ & 5.928-06 \\
\hline 1 & 20 & $5.783-05$ & $5.198-05$ & $4.350-05$ & $3.061-05$ & $1.705-05$ & $1.122-05$ & $8.125-06$ & $6.328-06$ & $4.451-06$ & 3.809-06 \\
\hline 1 & 21 & 7.476-04 & $7.428-04$ & $6.705-04$ & $4.863-04$ & 2.821-04 & $1.867-04$ & $1.299-04$ & $9.416-05$ & $5.144-05$ & $3.145-05$ \\
\hline 1 & 22 & 3.337-03 & $4.498-03$ & $5.812-03$ & $8.247-03$ & $1.262-02$ & $1.629-02$ & $1.956-02$ & $2.236-02$ & $2.788-02$ & $3.230-02$ \\
\hline 1 & 23 & 7.302-04 & $7.318-04$ & $6.623-04$ & $4.771-04$ & $2.738-04$ & $1.806-04$ & $1.250-04$ & $9.015-05$ & $4.905-05$ & $2.986-05$ \\
\hline 1 & 24 & 8.013-04 & $7.976-04$ & 7.353-04 & $5.772-04$ & $4.143-04$ & $3.508-04$ & $3.228-04$ & $3.121-04$ & $3.199-04$ & 3.409-04 \\
\hline 1 & 25 & 5.156-03 & $4.363-03$ & $3.490-03$ & $2.459-03$ & $1.406-03$ & 8.944-04 & $6.258-04$ & $4.614-04$ & $2.472-04$ & $1.498-04$ \\
\hline 1 & 26 & $3.500-03$ & $2.965-03$ & $2.357-03$ & $1.624-03$ & $8.924-04$ & $5.502-04$ & $3.748-04$ & $2.700-04$ & $1.389-04$ & $8.148-05$ \\
\hline 1 & 27 & $8.048-03$ & $7.763-03$ & $7.764-03$ & $8.454-03$ & $9.592-03$ & $1.044-02$ & $1.117-02$ & $1.193-02$ & $1.349-02$ & $1.390-02$ \\
\hline 1 & 28 & $2.383-03$ & $2.061-03$ & $1.661-03$ & $1.145-03$ & $6.310-04$ & $3.947-04$ & $2.707-04$ & $1.957-04$ & $1.019-04$ & $6.024-05$ \\
\hline 1 & 29 & 2.013-03 & $1.691-03$ & $1.330-03$ & $9.024-04$ & $4.787-04$ & $2.841-04$ & $1.876-04$ & $1.316-04$ & $6.418-05$ & $3.611-05$ \\
\hline 1 & 30 & $3.718-02$ & $3.586-02$ & $3.559-02$ & $3.708-02$ & $3.865-02$ & $3.918-02$ & $3.959-02$ & $3.984-02$ & $4.006-02$ & $4.016-02$ \\
\hline 1 & 31 & $4.873-03$ & $4.071-03$ & $3.250-03$ & $2.340-03$ & $1.384-03$ & 8.974-04 & $6.402-04$ & $4.802-04$ & $2.641-04$ & $1.636-04$ \\
\hline 1 & 32 & $2.959-03$ & $2.747-03$ & $2.555-03$ & $2.453-03$ & $2.451-03$ & $2.523-03$ & $2.624-03$ & $2.752-03$ & $3.046-03$ & $3.120-03$ \\
\hline 1 & 33 & $3.133-03$ & $2.706-03$ & $2.180-03$ & $1.502-03$ & $8.250-04$ & $5.126-04$ & $3.495-04$ & $2.516-04$ & $1.308-04$ & $7.765-05$ \\
\hline 1 & 34 & $3.495-03$ & $2.940-03$ & $2.323-03$ & $1.604-03$ & $9.011-04$ & $5.748-04$ & $4.056-04$ & $3.023-04$ & $1.666-04$ & $1.033-04$ \\
\hline 1 & 35 & $4.164-06$ & $3.807-06$ & $3.217-06$ & $2.282-06$ & $1.321-06$ & $8.898-07$ & $6.507-07$ & $5.067-07$ & $3.388-07$ & $2.591-07$ \\
\hline 1 & 36 & 5.871-04 & $5.077-04$ & $4.213-04$ & $3.240-04$ & $2.282-04$ & $1.842-04$ & $1.631-04$ & $1.511-04$ & $1.366-04$ & $1.305-04$ \\
\hline 1 & 37 & $2.189-05$ & $2.031-05$ & $1.720-05$ & $1.189-05$ & $6.528-06$ & $4.183-06$ & $2.867-06$ & $2.061-06$ & $1.098-06$ & $6.558-07$ \\
\hline 1 & 38 & 7.513-03 & $7.231-03$ & $6.097-03$ & $3.901-03$ & $1.839-03$ & $1.101-03$ & $6.994-04$ & $4.680-04$ & $2.387-04$ & $1.399-04$ \\
\hline 1 & 39 & $5.367-03$ & $6.102-03$ & $6.499-03$ & $6.728-03$ & $7.308-03$ & $7.818-03$ & 8.217-03 & $8.737-03$ & $1.003-02$ & $1.032-02$ \\
\hline 1 & 40 & $5.322-03$ & $5.327-03$ & $4.639-03$ & $3.049-03$ & $1.489-03$ & $9.220-04$ & $5.956-04$ & $4.028-04$ & $2.097-04$ & $1.243-04$ \\
\hline 1 & 41 & 2.934-03 & $2.773-03$ & $2.319-03$ & $1.494-03$ & $7.170-04$ & $4.305-04$ & $2.762-04$ & $1.872-04$ & $9.567-05$ & 5.595-05 \\
\hline 1 & 42 & $1.329-01$ & $1.528-01$ & $1.772-01$ & $2.205-01$ & $2.898-01$ & $3.444-01$ & $3.906-01$ & $4.306-01$ & $5.107-01$ & $5.727-01$ \\
\hline 1 & 43 & 7.865-03 & $7.750-03$ & $6.695-03$ & $4.405-03$ & $2.163-03$ & $1.335-03$ & 8.649-04 & $5.875-04$ & $3.054-04$ & $1.808-04$ \\
\hline 1 & 44 & $3.365-03$ & $3.474-03$ & $3.314-03$ & $2.886-03$ & $2.580-03$ & $2.548-03$ & $2.567-03$ & $2.659-03$ & $2.965-03$ & $3.022-03$ \\
\hline 1 & 45 & $1.207-03$ & $1.205-03$ & $1.014-03$ & 6.049-04 & $2.436-04$ & $1.347-04$ & $7.641-05$ & $4.484-05$ & $2.090-05$ & $1.193-05$ \\
\hline 1 & 46 & $1.002-02$ & $1.131-02$ & $1.270-02$ & $1.506-02$ & $1.901-02$ & $2.225-02$ & $2.502-02$ & $2.743-02$ & $3.231-02$ & 3.609-02 \\
\hline 1 & 47 & $\begin{array}{l}\text { 7.397-04 } \\
\text { available on }\end{array}$ & $\begin{array}{l}7.381-04 \\
\text { request }\end{array}$ & $6.473-04$ & $4.371-04$ & $2.234-04$ & $1.414-04$ & $9.360-05$ & $6.483-05$ & $3.451-05$ & $2.078-05$ \\
\hline 20 & 298 & $3.374-08$ & $4.069-08$ & $4.920-08$ & $6.386-08$ & $8.450-08$ & $9.963-08$ & $1.101-07$ & $1.183-07$ & $1.338-07$ & $1.449-07$ \\
\hline 20 & 300 & $3.965-06$ & $3.357-06$ & $2.807-06$ & 2.091-06 & $1.270-06$ & $8.506-07$ & 6.059-07 & $4.517-07$ & $2.499-07$ & $1.565-07$ \\
\hline 20 & 302 & $5.059-10$ & $4.350-10$ & $3.896-10$ & $4.263-10$ & $5.305-10$ & $5.849-10$ & $6.062-10$ & $6.130-10$ & $6.062-10$ & $6.248-10$ \\
\hline 20 & 303 & $6.655-06$ & $5.632-06$ & $4.710-06$ & $3.511-06$ & $2.137-06$ & $1.433-06$ & $1.024-06$ & $7.667-07$ & $4.308-07$ & $2.760-07$ \\
\hline 20 & 304 & $1.369-08$ & $1.634-08$ & $1.968-08$ & $2.557-08$ & $3.421-08$ & $4.070-08$ & $4.534-08$ & $4.901-08$ & $5.600-08$ & $6.114-08$ \\
\hline 20 & 305 & 4.971-10 & $3.656-10$ & $2.607-10$ & $1.477-10$ & $6.542-11$ & $3.696-11$ & $2.310-11$ & $1.532-11$ & $6.607-12$ & $3.374-12$ \\
\hline 20 & 306 & $9.442-06$ & $7.989-06$ & $6.679-06$ & $4.974-06$ & $3.022-06$ & $2.024-06$ & $1.442-06$ & $1.075-06$ & 5.949-07 & $3.725-07$ \\
\hline 20 & 310 & 3.921-10 & $2.672-10$ & $1.758-10$ & $8.783-11$ & $3.385-11$ & $1.773-11$ & $1.056-11$ & $6.796-12$ & $2.811-12$ & $1.425-12$ \\
\hline 20 & 311 & $3.520-10$ & $2.460-10$ & $1.706-10$ & $1.214-10$ & $1.171-10$ & $1.191-10$ & $1.171-10$ & $1.146-10$ & $1.117-10$ & $1.156-10$ \\
\hline 20 & 312 & 8.861-10 & $6.400-10$ & $4.484-10$ & $2.467-10$ & $1.023-10$ & $5.445-11$ & $3.308-11$ & $2.173-11$ & $9.329-12$ & 4.913-12 \\
\hline 20 & 313 & $8.908-10$ & $7.059-10$ & $5.833-10$ & $5.502-10$ & $5.888-10$ & $6.542-10$ & $7.155-10$ & $7.030-10$ & $8.622-10$ & $1.191-09$ \\
\hline 20 & 314 & $9.768-10$ & $7.346-10$ & $5.447-10$ & $3.327-10$ & $1.604-10$ & $9.214-11$ & $5.790-11$ & $3.853-11$ & $1.654-11$ & 8.508-12 \\
\hline
\end{tabular}


Table VII-continued ...

\begin{tabular}{|c|c|c|c|c|c|c|c|c|c|c|c|}
\hline \multirow[t]{2}{*}{$\bar{i}$} & \multirow[t]{2}{*}{$\mathrm{j}$} & \multicolumn{10}{|c|}{$\begin{array}{c}\text { Collision strength } \Omega \\
\text { Scattered electron energy }(\mathrm{eV})\end{array}$} \\
\hline & & 10 & 50 & 100 & 200 & 400 & 600 & 800 & 1000 & 1500 & 2000 \\
\hline 20 & 316 & $3.195-06$ & $2.610-06$ & $2.119-06$ & $1.469-06$ & $8.346-07$ & $5.296-07$ & $3.637-07$ & $2.635-07$ & $1.347-07$ & $7.872-08$ \\
\hline 20 & 317 & $1.792-05$ & $1.855-05$ & $1.972-05$ & $2.215-05$ & $2.695-05$ & $3.150-05$ & $3.561-05$ & $3.945-05$ & $4.775-05$ & $5.416-05$ \\
\hline 20 & 319 & $1.725-05$ & $1.415-05$ & $1.154-05$ & $8.048-06$ & $4.606-06$ & 2.934-06 & 2.021-06 & $1.468-06$ & 7.553-07 & $4.443-07$ \\
\hline 20 & 321 & $4.165-05$ & 4.898-05 & $5.759-05$ & $7.249-05$ & $9.655-05$ & $1.168-04$ & $1.341-04$ & $1.499-04$ & $1.832-04$ & 2.084-04 \\
\hline 20 & 322 & $1.846-05$ & $1.488-05$ & $1.178-05$ & $8.045-06$ & $4.340-06$ & $2.703-06$ & $1.824-06$ & $1.300-06$ & $6.629-07$ & $3.893-07$ \\
\hline 20 & 324 & $3.274-05$ & $2.674-05$ & $2.163-05$ & $1.567-05$ & $9.947-06$ & $7.578-06$ & $6.378-06$ & $5.704-06$ & 4.964-06 & $4.678-06$ \\
\hline 20 & 326 & 2.097-05 & $1.773-05$ & $1.460-05$ & $1.050-05$ & 5.995-06 & $3.859-06$ & $2.671-06$ & $1.946-06$ & $1.033-06$ & $6.268-07$ \\
\hline 20 & 328 & $3.852-05$ & $3.778-05$ & $3.745-05$ & $3.767-05$ & $3.795-05$ & $3.830-05$ & 3.856-05 & $3.875-05$ & 3.906-05 & $3.921-05$ \\
\hline 20 & 330 & $4.741-05$ & $3.786-05$ & 2.977-05 & $2.021-05$ & $1.089-05$ & $6.803-06$ & $4.602-06$ & $3.285-06$ & $1.676-06$ & $9.868-07$ \\
\hline 20 & 331 & $2.457-05$ & 2.094-05 & $1.737-05$ & $1.262-05$ & 7.294-06 & $4.736-06$ & $3.300-06$ & $2.416-06$ & $1.293-06$ & 7.892-07 \\
\hline 20 & 332 & $2.461-05$ & $2.101-05$ & $1.745-05$ & $1.268-05$ & 7.327-06 & $4.753-06$ & $3.310-06$ & $2.424-06$ & $1.299-06$ & $7.922-07$ \\
\hline 20 & 333 & $2.630-05$ & $2.227-05$ & $1.869-05$ & $1.450-05$ & $1.057-05$ & $9.100-06$ & $8.511-06$ & $8.275-06$ & $8.183-06$ & $8.223-06$ \\
\hline 20 & 334 & $3.267-05$ & $3.060-05$ & $2.893-05$ & $2.777-05$ & $2.796-05$ & $2.905-05$ & $3.038-05$ & $3.161-05$ & $3.387-05$ & $3.526-05$ \\
\hline 20 & 335 & 3.893-05 & $2.945-05$ & $2.209-05$ & $1.379-05$ & $6.531-06$ & $3.741-06$ & $2.389-06$ & $1.644-06$ & $8.025-07$ & $4.635-07$ \\
\hline 20 & 337 & $5.269-05$ & $4.015-05$ & $3.050-05$ & $1.987-05$ & $1.090-05$ & $7.683-06$ & $6.262-06$ & $5.537-06$ & $4.885-06$ & $4.834-06$ \\
\hline 20 & 339 & $4.323-04$ & $4.322-04$ & $4.366-04$ & $4.498-04$ & $4.638-04$ & $4.727-04$ & $4.782-04$ & 4.819-04 & $4.872-04$ & 4.897-04 \\
\hline 20 & 341 & $6.429-05$ & 4.916-05 & $3.746-05$ & $2.396-05$ & $1.177-05$ & $6.927-06$ & $4.513-06$ & $3.151-06$ & $1.566-06$ & $9.133-07$ \\
\hline 20 & 342 & $1.689-06$ & $1.397-06$ & $1.113-06$ & $7.585-07$ & $4.088-07$ & $2.552-07$ & $1.727-07$ & $1.237-07$ & $6.430-08$ & $3.836-08$ \\
\hline 20 & 343 & 2.021-04 & $2.157-04$ & $2.344-04$ & $2.675-04$ & $3.188-04$ & $3.601-04$ & $3.945-04$ & $4.239-04$ & 4.836-04 & 5.307-04 \\
\hline 20 & 344 & $3.870-06$ & $3.238-06$ & $2.626-06$ & $1.851-06$ & $1.086-06$ & 7.541-07 & $5.815-07$ & $4.811-07$ & $3.630-07$ & $3.190-07$ \\
\hline 20 & 345 & $6.712-06$ & $5.600-06$ & $4.514-06$ & $3.129-06$ & $1.717-06$ & $1.083-06$ & 7.389-07 & $5.330-07$ & $2.800-07$ & $1.677-07$ \\
\hline 20 & 346 & 8.103-05 & $6.170-05$ & $4.661-05$ & 2.944-05 & $1.423-05$ & $8.274-06$ & $5.347-06$ & $3.715-06$ & $1.836-06$ & $1.064-06$ \\
\hline 20 & 347 & $4.842-05$ & $3.648-05$ & $2.729-05$ & $1.693-05$ & 7.909-06 & $4.485-06$ & $2.839-06$ & $1.939-06$ & $9.346-07$ & $5.392-07$ \\
\hline 20 & 349 & $3.754-05$ & $2.826-05$ & $2.125-05$ & $1.366-05$ & 7.544-06 & $5.467-06$ & $4.598-06$ & 4.197-06 & $3.842-06$ & $3.677-06$ \\
\hline 20 & 351 & $2.358-06$ & $1.481-06$ & $9.240-07$ & 4.369-07 & $1.540-07$ & 7.264-08 & $3.982-08$ & $2.410-08$ & 8.990-09 & 4.346-09 \\
\hline 20 & 352 & $4.833-06$ & $3.062-06$ & $1.898-06$ & $8.557-07$ & $2.747-07$ & $1.195-07$ & $6.038-08$ & $3.373-08$ & $1.018-08$ & 3.930-09 \\
\hline 20 & 353 & $1.862-05$ & $1.394-05$ & $1.050-05$ & 6.908-06 & $4.205-06$ & $3.281-06$ & 2.914-06 & $2.780-06$ & $2.708-06$ & $2.528-06$ \\
\hline 20 & 354 & $2.717-06$ & $1.782-06$ & $1.206-06$ & 7.599-07 & $5.590-07$ & $5.620-07$ & $5.521-07$ & $5.408-07$ & $5.436-07$ & $5.722-07$ \\
\hline 20 & 355 & $8.617-05$ & $6.635-05$ & $5.095-05$ & $3.300-05$ & $1.653-05$ & $9.865-06$ & 6.494-06 & $4.569-06$ & 2.297-06 & $1.346-06$ \\
\hline 20 & 356 & 7.203-05 & $5.723-05$ & $4.584-05$ & $3.264-05$ & $2.077-05$ & $1.624-05$ & $1.423-05$ & $1.328-05$ & $1.270-05$ & $1.291-05$ \\
\hline 20 & 357 & $2.742-05$ & $2.138-05$ & $1.665-05$ & $1.103-05$ & 5.709-06 & $3.483-06$ & $2.329-06$ & $1.658-06$ & 8.466-07 & $4.985-07$ \\
\hline 20 & 358 & $1.845-05$ & $1.275-05$ & 8.674-06 & $4.632-06$ & $1.871-06$ & $9.837-07$ & $5.908-07$ & $3.865-07$ & $1.688-07$ & 8.893-08 \\
\hline 20 & 360 & $2.186-05$ & $1.587-05$ & $1.186-05$ & 8.194-06 & $6.279-06$ & $6.032-06$ & $6.081-06$ & $6.225-06$ & $6.604-06$ & $6.807-06$ \\
\hline 20 & 361 & $3.060-05$ & $2.127-05$ & $1.454-05$ & $7.720-06$ & $3.016-06$ & $1.520-06$ & 8.839-07 & $5.639-07$ & $2.277-07$ & $1.093-07$ \\
\hline 20 & 362 & $3.192-05$ & $3.148-05$ & $3.272-05$ & $3.672-05$ & $4.307-05$ & $4.785-05$ & 5.099-05 & $5.305-05$ & $5.583-05$ & $5.743-05$ \\
\hline 20 & 363 & $3.831-05$ & $4.306-05$ & $4.935-05$ & $6.259-05$ & $8.739-05$ & $1.095-04$ & $1.268-04$ & $1.393-04$ & $1.592-04$ & $1.724-04$ \\
\hline 20 & 364 & 2.894-05 & $2.502-05$ & $2.306-05$ & $2.346-05$ & $2.803-05$ & $3.242-05$ & $3.592-05$ & $3.856-05$ & $4.368-05$ & 4.807-05 \\
\hline 20 & 365 & $1.249-03$ & $1.391-03$ & $1.558-03$ & $1.834-03$ & $2.235-03$ & $2.542-03$ & $2.793-03$ & $3.006-03$ & $3.434-03$ & $3.770-03$ \\
\hline 20 & 367 & $2.221-06$ & $1.355-06$ & $8.139-07$ & $3.585-07$ & $1.152-07$ & $5.348-08$ & $2.951-08$ & $1.808-08$ & 6.873-09 & $3.225-09$ \\
\hline 20 & 368 & $2.645-06$ & $1.652-06$ & $1.035-06$ & $5.465-07$ & $3.134-07$ & $2.854-07$ & $2.712-07$ & $2.618-07$ & $2.578-07$ & $2.675-07$ \\
\hline 20 & 369 & $5.501-06$ & $4.792-06$ & $4.460-06$ & $4.374-06$ & $4.715-06$ & $4.937-06$ & $5.133-06$ & $5.324-06$ & $5.614-06$ & $5.498-06$ \\
\hline 20 & 370 & $5.486-06$ & $3.518-06$ & $2.213-06$ & $1.027-06$ & $3.466-07$ & $1.573-07$ & $8.299-08$ & $4.833-08$ & $1.583-08$ & $6.445-09$ \\
\hline 20 & 371 & $4.238-06$ & $2.720-06$ & $1.743-06$ & $8.629-07$ & $3.199-07$ & $1.525-07$ & $8.340-08$ & $5.021-08$ & $1.870-08$ & $9.152-09$ \\
\hline 20 & 372 & $3.281-06$ & $2.273-06$ & $1.650-06$ & $1.203-06$ & $1.044-06$ & $1.113-06$ & $1.122-06$ & $1.115-06$ & $1.133-06$ & $1.188-06$ \\
\hline 20 & 373 & $5.090-06$ & $3.330-06$ & $2.137-06$ & $1.023-06$ & $3.581-07$ & $1.640-07$ & 8.659-08 & 5.034-08 & $1.640-08$ & 6.737-09 \\
\hline 20 & 374 & $9.023-06$ & $7.962-06$ & $7.435-06$ & $7.256-06$ & $7.780-06$ & $8.131-06$ & $8.447-06$ & $8.758-06$ & $9.233-06$ & $9.055-06$ \\
\hline 20 & 375 & $1.801-05$ & $1.211-05$ & 7.971-06 & $3.997-06$ & $1.452-06$ & 7.184-07 & $4.214-07$ & $2.756-07$ & $1.233-07$ & $6.581-08$ \\
\hline 20 & 376 & 2.354-05 & $1.595-05$ & $1.065-05$ & $5.483-06$ & $2.195-06$ & $1.254-06$ & $8.678-07$ & $6.813-07$ & $5.008-07$ & $4.384-07$ \\
\hline 20 & 377 & $3.431-05$ & 2.409-05 & $1.667-05$ & $9.184-06$ & 3.881-06 & $2.087-06$ & $1.264-06$ & $8.275-07$ & 3.584-07 & $1.885-07$ \\
\hline 20 & 378 & $4.088-05$ & $2.869-05$ & $1.982-05$ & $1.070-05$ & $4.272-06$ & $2.174-06$ & $1.267-06$ & $8.056-07$ & $3.209-07$ & $1.525-07$ \\
\hline 20 & 379 & $2.242-05$ & $1.810-05$ & $1.576-05$ & $1.438-05$ & $1.504-05$ & $1.629-05$ & $1.732-05$ & $1.822-05$ & $1.986-05$ & $2.065-05$ \\
\hline 20 & 380 & $4.582-05$ & $4.408-05$ & $4.479-05$ & $4.913-05$ & $5.737-05$ & 6.404-05 & $6.860-05$ & $7.172-05$ & 7.607-05 & $7.761-05$ \\
\hline 20 & 381 & $2.504-05$ & $1.779-05$ & $1.246-05$ & $6.867-06$ & $2.818-06$ & $1.452-06$ & $8.482-07$ & $5.379-07$ & $2.110-07$ & 9.884-08 \\
\hline 20 & 382 & 8.355-05 & $9.158-05$ & $1.028-04$ & $1.240-04$ & $1.529-04$ & $1.732-04$ & $1.865-04$ & $1.955-04$ & $2.078-04$ & $2.122-04$ \\
\hline 20 & 383 & $1.831-06$ & $1.319-06$ & $9.302-07$ & $5.304-07$ & $2.360-07$ & $1.287-07$ & 7.804-08 & $5.133-08$ & $2.364-08$ & $1.340-08$ \\
\hline 20 & 384 & $2.579-06$ & $1.881-06$ & $1.342-06$ & $7.849-07$ & $3.952-07$ & $2.609-07$ & $2.045-07$ & $1.728-07$ & $1.345-07$ & $1.330-07$ \\
\hline 20 & 385 & $3.356-06$ & $2.441-06$ & $1.738-06$ & $1.007-06$ & $4.547-07$ & $2.501-07$ & $1.531-07$ & $1.017-07$ & $4.802-08$ & $2.764-08$ \\
\hline 20 & 386 & $8.717-07$ & $5.872-07$ & $3.980-07$ & $2.316-07$ & $1.568-07$ & $1.562-07$ & $1.677-07$ & $1.811-07$ & $2.135-07$ & $2.428-07$ \\
\hline 20 & 387 & $1.358-06$ & $8.732-07$ & $5.449-07$ & $2.445-07$ & 8.037-08 & $4.180-08$ & $2.725-08$ & $1.895-08$ & 7.269-09 & 3.105-09 \\
\hline
\end{tabular}


Table VII-continued ...

\begin{tabular}{|c|c|c|c|c|c|c|c|c|c|c|c|}
\hline \multirow[t]{2}{*}{$\overline{\mathrm{i}}$} & \multirow[t]{2}{*}{$\mathrm{j}$} & \multicolumn{10}{|c|}{$\begin{array}{c}\text { Collision strength } \Omega \\
\text { Scattered electron energy }(\mathrm{eV})\end{array}$} \\
\hline & & 10 & 50 & 100 & 200 & 400 & 600 & 800 & 1000 & 1500 & 2000 \\
\hline 20 & 388 & $2.020-06$ & $1.480-06$ & $1.254-06$ & $1.331-06$ & $2.062-06$ & $2.655-06$ & $3.147-06$ & $3.318-06$ & $3.337-06$ & $3.813-06$ \\
\hline 20 & 389 & $2.868-06$ & $3.289-06$ & $4.671-06$ & $8.196-06$ & $1.552-05$ & $2.052-05$ & $2.452-05$ & $2.597-05$ & $2.628-05$ & $3.005-05$ \\
\hline 20 & 390 & $1.435-06$ & $9.306-07$ & $5.866-07$ & $2.681-07$ & $9.080-08$ & $4.752-08$ & $3.080-08$ & $2.129-08$ & 8.184-09 & 3.641-09 \\
\hline 20 & 391 & 3.854-06 & $2.786-06$ & $1.979-06$ & $1.136-06$ & $5.417-07$ & $3.212-07$ & $2.154-07$ & $1.562-07$ & 8.514-08 & $5.473-08$ \\
\hline 20 & 392 & $2.453-06$ & $1.824-06$ & $1.356-06$ & $8.870-07$ & $6.115-07$ & $5.703-07$ & 5.988-07 & $6.553-07$ & $8.278-07$ & $9.867-07$ \\
\hline 20 & 393 & 7.668-07 & $5.538-07$ & 3.923-07 & $2.235-07$ & $1.054-07$ & $6.159-08$ & $4.084-08$ & $2.942-08$ & $1.589-08$ & $1.014-08$ \\
\hline 20 & 395 & $1.241-04$ & $1.343-04$ & $1.493-04$ & $1.817-04$ & $2.556-04$ & 3.389-04 & $4.255-04$ & 5.113-04 & $7.110-04$ & 8.795-04 \\
\hline 20 & 397 & $4.359-06$ & $3.027-06$ & 2.059-06 & $1.121-06$ & $4.573-07$ & $2.413-07$ & $1.468-07$ & $9.723-08$ & $4.277-08$ & $2.263-08$ \\
\hline 20 & 399 & 5.629-06 & $3.892-06$ & $2.630-06$ & $1.443-06$ & $5.841-07$ & $3.063-07$ & $1.895-07$ & $1.276-07$ & $5.618-08$ & $3.312-08$ \\
\hline 20 & 400 & 6.934-06 & $4.802-06$ & $3.254-06$ & $1.763-06$ & $7.121-07$ & $3.733-07$ & $2.261-07$ & $1.494-07$ & $6.552-08$ & $3.464-08$ \\
\hline 20 & 401 & $1.559-06$ & $1.033-06$ & $6.559-07$ & $3.074-07$ & $1.006-07$ & 4.607-08 & 2.709-08 & $1.864-08$ & $1.156-08$ & 5.158-09 \\
\hline 20 & 403 & $2.851-06$ & $1.888-06$ & $1.201-06$ & $5.680-07$ & $1.932-07$ & $9.367-08$ & 5.928-08 & $4.390-08$ & $2.963-08$ & $3.088-08$ \\
\hline 20 & 405 & 2.197-06 & $1.455-06$ & $9.278-07$ & $4.335-07$ & $1.424-07$ & $6.353-08$ & $3.550-08$ & $2.357-08$ & $1.191-08$ & 6.783-09 \\
\hline 20 & 407 & $2.121-06$ & $1.519-06$ & $1.050-06$ & $5.683-07$ & $2.329-07$ & $1.195-07$ & $6.883-08$ & $4.332-08$ & $1.826-08$ & 9.643-09 \\
\hline 20 & 408 & $3.648-06$ & $2.620-06$ & $1.817-06$ & $9.937-07$ & $4.107-07$ & $2.141-07$ & $1.279-07$ & $8.303-08$ & $3.552-08$ & $2.451-08$ \\
\hline 20 & 409 & $5.368-06$ & $3.868-06$ & $2.700-06$ & $1.490-06$ & $6.270-07$ & $3.305-07$ & $1.958-07$ & $1.262-07$ & $5.498-08$ & $3.008-08$ \\
\hline 20 & 410 & $1.943-05$ & $1.538-05$ & $1.202-05$ & $8.117-06$ & $4.389-06$ & $2.796-06$ & $1.953-06$ & $1.442-06$ & 7.747-07 & $4.626-07$ \\
\hline 20 & 412 & $2.374-06$ & $1.593-06$ & $1.030-06$ & $4.926-07$ & $1.677-07$ & 7.864-08 & $4.584-08$ & $3.120-08$ & $1.618-08$ & $9.208-09$ \\
\hline 20 & 414 & $2.573-06$ & 2.921-06 & $4.131-06$ & $6.403-06$ & $1.046-05$ & $1.241-05$ & $1.306-05$ & $1.261-05$ & $1.353-05$ & $1.985-05$ \\
\hline 20 & 415 & $8.143-07$ & $6.228-07$ & 4.699-07 & $3.119-07$ & $2.071-07$ & $1.778-07$ & $1.677-07$ & $1.631-07$ & $1.576-07$ & $1.554-07$ \\
\hline 20 & 416 & 2.005-06 & $1.430-06$ & $9.692-07$ & $4.902-07$ & $1.792-07$ & $9.092-08$ & $5.690-08$ & $4.047-08$ & 2.169-08 & $1.298-08$ \\
\hline 20 & 418 & $3.440-06$ & $2.458-06$ & $1.670-06$ & $8.573-07$ & $3.192-07$ & $1.810-07$ & $1.345-07$ & $1.125-07$ & $1.126-07$ & $1.425-07$ \\
\hline 20 & 419 & $3.766-06$ & $3.407-06$ & $3.331-06$ & $4.302-06$ & $1.078-05$ & $1.935-05$ & 2.799-05 & $3.273-05$ & 5.819-05 & $1.004-04$ \\
\hline 20 & 421 & $1.246-06$ & $8.300-07$ & $5.227-07$ & $2.667-07$ & $1.320-07$ & 7.315-08 & $4.208-08$ & $2.688-08$ & $1.484-08$ & $1.324-08$ \\
\hline 20 & 423 & $1.613-06$ & $1.063-06$ & $6.740-07$ & $3.395-07$ & $1.615-07$ & $8.543-08$ & $4.705-08$ & $2.709-08$ & 8.296-09 & $3.608-09$ \\
\hline 20 & 424 & $1.997-06$ & $1.308-06$ & $8.470-07$ & $4.151-07$ & $1.875-07$ & $1.090-07$ & $6.008-08$ & $3.185-08$ & 9.954-09 & 3.991-09 \\
\hline 20 & 425 & $4.316-06$ & $3.615-06$ & 2.966-06 & 2.151-06 & $1.239-06$ & 7.913-07 & $5.381-07$ & $3.828-07$ & $1.897-07$ & $1.068-07$ \\
\hline 20 & 426 & $1.807-06$ & $1.366-06$ & $9.792-07$ & $5.228-07$ & $1.836-07$ & $8.597-08$ & $4.792-08$ & $2.956-08$ & $1.158-08$ & 5.465-09 \\
\hline 20 & 428 & $2.552-06$ & $1.951-06$ & $1.411-06$ & 7.363-07 & $2.885-07$ & $1.316-07$ & $8.564-08$ & $6.705-08$ & $4.774-08$ & $5.193-08$ \\
\hline 20 & 429 & $3.253-06$ & $2.441-06$ & $1.752-06$ & $9.405-07$ & $3.330-07$ & $1.576-07$ & $8.768-08$ & $5.401-08$ & $2.125-08$ & $9.892-09$ \\
\hline 20 & 430 & $5.610-05$ & 5.416-05 & 5.293-05 & 5.341-05 & $6.009-05$ & 6.988-05 & $7.922-05$ & $8.762-05$ & $1.037-04$ & $1.148-04$ \\
\hline 20 & 431 & $1.664-06$ & $1.105-06$ & $7.082-07$ & $3.601-07$ & $1.645-07$ & 8.769-08 & 4.831-08 & $2.756-08$ & 8.854-09 & 3.818-09 \\
\hline 20 & 432 & $9.403-06$ & $1.059-05$ & $1.188-05$ & $1.385-05$ & $1.666-05$ & $1.828-05$ & $1.945-05$ & $1.916-05$ & $2.362-05$ & $3.147-05$ \\
\hline 20 & 433 & $7.792-05$ & $6.413-05$ & 5.164-05 & $3.638-05$ & $2.081-05$ & $1.365-05$ & $9.663-06$ & $7.185-06$ & 3.929-06 & $2.435-06$ \\
\hline 20 & 434 & $5.759-06$ & $4.004-06$ & $2.776-06$ & $1.545-06$ & $5.714-07$ & $2.660-07$ & $1.425-07$ & 8.348-08 & $2.978-08$ & $1.362-08$ \\
\hline 20 & 435 & $6.947-06$ & 4.998-06 & $3.537-06$ & $1.876-06$ & $6.970-07$ & $3.327-07$ & $1.784-07$ & $1.037-07$ & $3.847-08$ & $1.967-08$ \\
\hline 20 & 436 & 8.393-06 & $5.837-06$ & $4.048-06$ & $2.256-06$ & $8.302-07$ & $3.868-07$ & $2.072-07$ & $1.210-07$ & 4.331-08 & $1.975-08$ \\
\hline 20 & 437 & $1.518-06$ & $1.113-06$ & $7.744-07$ & $4.362-07$ & $2.112-07$ & $1.275-07$ & $8.000-08$ & $5.103-08$ & $1.832-08$ & 7.357-09 \\
\hline 20 & 438 & $9.118-07$ & 6.684-07 & $4.690-07$ & 2.691-07 & $1.342-07$ & $8.546-08$ & $5.729-08$ & $4.002-08$ & $2.252-08$ & $1.842-08$ \\
\hline 20 & 439 & 2.116-06 & $1.565-06$ & $1.093-06$ & $6.065-07$ & $3.111-07$ & $1.938-07$ & $1.137-07$ & $6.681-08$ & 2.921-08 & $1.594-08$ \\
\hline 20 & 440 & $1.664-06$ & $1.214-06$ & $8.410-07$ & $4.701-07$ & $2.226-07$ & $1.329-07$ & $8.362-08$ & $5.372-08$ & $1.975-08$ & 8.187-09 \\
\hline 20 & 441 & $2.052-06$ & $2.639-06$ & $3.039-06$ & $5.032-06$ & $7.446-06$ & $8.501-06$ & 8.936-06 & $9.078-06$ & 8.890-06 & $9.111-06$ \\
\hline 20 & 442 & $1.086-06$ & $9.239-07$ & $7.352-07$ & $4.662-07$ & $2.069-07$ & $1.019-07$ & $5.359-08$ & $2.963-08$ & 8.445-09 & 2.992-09 \\
\hline 20 & 443 & $3.342-06$ & $2.883-06$ & $2.358-06$ & $1.619-06$ & $9.429-07$ & $6.960-07$ & 5.999-07 & $5.641-07$ & $5.642-07$ & 5.917-07 \\
\hline 20 & 444 & $5.374-06$ & $4.563-06$ & $3.636-06$ & 2.309-06 & $1.024-06$ & $5.040-07$ & 2.653-07 & $1.473-07$ & $4.213-08$ & $1.500-08$ \\
\hline 20 & 445 & $1.733-04$ & $2.224-04$ & $2.857-04$ & $4.009-04$ & 5.686-04 & 6.921-04 & $7.745-04$ & 8.381-04 & $9.565-04$ & $1.036-03$ \\
\hline 20 & 446 & $1.478-03$ & $1.507-03$ & $1.562-03$ & $1.682-03$ & $1.857-03$ & $1.982-03$ & $2.071-03$ & $2.138-03$ & $2.251-03$ & 2.324-03 \\
\hline 20 & 447 & $2.269-06$ & $1.843-06$ & $1.489-06$ & $1.026-06$ & $5.796-07$ & $3.666-07$ & $2.505-07$ & $1.801-07$ & $8.950-08$ & $5.044-08$ \\
\hline 20 & 448 & $8.340-06$ & $8.380-06$ & $8.701-06$ & $9.558-06$ & $1.143-05$ & $1.308-05$ & $1.438-05$ & $1.552-05$ & $1.780-05$ & $1.946-05$ \\
\hline 20 & 449 & $1.365-05$ & $1.114-05$ & $9.031-06$ & 6.249-06 & $3.550-06$ & $2.252-06$ & $1.541-06$ & $1.109-06$ & $5.520-07$ & $3.123-07$ \\
\hline 20 & 450 & $1.129-05$ & $1.293-05$ & $1.496-05$ & $1.867-05$ & $2.478-05$ & 2.954-05 & $3.313-05$ & $3.616-05$ & $4.215-05$ & 4.643-05 \\
\hline 20 & 451 & $5.123-06$ & $4.147-06$ & $3.290-06$ & $2.243-06$ & $1.200-06$ & 7.414-07 & $4.980-07$ & $3.546-07$ & $1.809-07$ & $1.056-07$ \\
\hline 20 & 452 & $5.484-06$ & $4.447-06$ & $3.535-06$ & 2.419-06 & $1.305-06$ & $8.125-07$ & $5.500-07$ & $3.940-07$ & $2.031-07$ & $1.201-07$ \\
\hline 20 & 453 & $1.491-05$ & $1.307-05$ & $1.153-05$ & $9.848-06$ & $8.428-06$ & 7.979-06 & $7.828-06$ & $7.815-06$ & 7.937-06 & $8.026-06$ \\
\hline 20 & 454 & $1.005-06$ & $9.619-07$ & $9.855-07$ & $1.182-06$ & $1.772-06$ & $2.360-06$ & $2.820-06$ & $3.175-06$ & $3.750-06$ & 4.049-06 \\
\hline 20 & 455 & $3.052-06$ & $2.476-06$ & $1.965-06$ & $1.339-06$ & 7.104-07 & $4.351-07$ & $2.901-07$ & $2.051-07$ & $1.035-07$ & $6.055-08$ \\
\hline 20 & 456 & $8.095-06$ & $7.021-06$ & $5.930-06$ & $4.420-06$ & $2.642-06$ & $1.755-06$ & $1.245-06$ & $9.232-07$ & $5.043-07$ & $3.111-07$ \\
\hline 20 & 457 & $1.048-04$ & $1.052-04$ & $1.069-04$ & $1.110-04$ & $1.155-04$ & $1.183-04$ & $1.201-04$ & $1.214-04$ & $1.237-04$ & $1.251-04$ \\
\hline 20 & 458 & $4.564-06$ & $3.688-06$ & 2.918-06 & $1.989-06$ & $1.083-06$ & $7.048-07$ & $5.140-07$ & $4.079-07$ & 2.897-07 & $2.389-07$ \\
\hline
\end{tabular}


Table VII-continued ...

\begin{tabular}{|c|c|c|c|c|c|c|c|c|c|c|c|}
\hline \multirow[t]{2}{*}{$\overline{\mathrm{i}}$} & \multirow[t]{2}{*}{$\mathrm{j}$} & \multicolumn{10}{|c|}{$\begin{array}{c}\text { Collision strength } \Omega \\
\text { Scattered electron energy }(\mathrm{eV})\end{array}$} \\
\hline & & 10 & 50 & 100 & 200 & 400 & 600 & 800 & 1000 & 1500 & 2000 \\
\hline 20 & 459 & $2.163-05$ & $1.739-05$ & $1.373-05$ & $9.331-06$ & $5.010-06$ & $3.121-06$ & $2.110-06$ & $1.506-06$ & $7.682-07$ & $4.490-07$ \\
\hline 20 & 460 & $2.912-05$ & $2.322-05$ & $1.821-05$ & $1.204-05$ & $6.468-06$ & $3.970-06$ & $2.640-06$ & $1.847-06$ & $8.625-07$ & 4.599-07 \\
\hline 20 & 461 & $2.168-05$ & $2.132-05$ & $2.126-05$ & 2.189-05 & $2.349-05$ & $2.490-05$ & 2.599-05 & 2.693-05 & 2.867-05 & 2.957-05 \\
\hline 20 & 462 & $1.302-04$ & $1.211-04$ & $1.161-04$ & $1.151-04$ & $1.264-04$ & $1.422-04$ & $1.581-04$ & $1.734-04$ & $2.075-04$ & $2.348-04$ \\
\hline 20 & 463 & $1.348-04$ & $1.059-04$ & 8.203-05 & 5.317-05 & $2.771-05$ & $1.670-05$ & $1.096-05$ & 7.593-06 & $3.461-06$ & $1.792-06$ \\
\hline 20 & 464 & $6.511-06$ & $5.471-06$ & $4.491-06$ & $3.216-06$ & $1.823-06$ & $1.166-06$ & 8.036-07 & $5.842-07$ & $3.091-07$ & $1.859-07$ \\
\hline 20 & 465 & $2.383-05$ & $1.850-05$ & $1.419-05$ & $9.125-06$ & $4.608-06$ & $2.738-06$ & $1.787-06$ & $1.241-06$ & 5.929-07 & $3.294-07$ \\
\hline 20 & 466 & $1.800-05$ & $1.381-05$ & $1.062-05$ & $7.226-06$ & $4.555-06$ & $3.724-06$ & $3.426-06$ & $3.322-06$ & $3.349-06$ & $3.510-06$ \\
\hline 20 & 467 & $2.652-05$ & $2.221-05$ & $1.823-05$ & $1.306-05$ & $7.580-06$ & 4.905-06 & 3.394-06 & $2.467-06$ & $1.302-06$ & 7.969-07 \\
\hline 20 & 468 & $3.051-04$ & $3.549-04$ & 4.134-04 & 5.134-04 & $6.713-04$ & 7.996-04 & $9.066-04$ & $1.000-03$ & $1.194-03$ & $1.344-03$ \\
\hline 20 & 469 & $9.648-06$ & $8.011-06$ & $6.553-06$ & 4.779-06 & $2.968-06$ & $2.186-06$ & $1.796-06$ & $1.587-06$ & $1.377-06$ & $1.316-06$ \\
\hline 20 & 471 & $1.608-04$ & $1.601-04$ & $1.610-04$ & $1.646-04$ & $1.671-04$ & $1.683-04$ & $1.689-04$ & $1.693-04$ & $1.704-04$ & $1.712-04$ \\
\hline 20 & 472 & $3.650-04$ & 4.569-04 & $5.655-04$ & 7.581-04 & $1.087-03$ & $1.371-03$ & $1.621-03$ & $1.849-03$ & 2.334-03 & $2.705-03$ \\
\hline 20 & 474 & $5.114-08$ & $2.851-08$ & $1.512-08$ & $5.610-09$ & $1.305-09$ & $4.880-10$ & $2.256-10$ & $1.169-10$ & $3.934-11$ & $1.831-11$ \\
\hline 20 & 475 & $1.085-07$ & $6.070-08$ & $3.251-08$ & $1.209-08$ & 2.961-09 & 1.019-09 & $4.114-10$ & $1.920-10$ & 3.995-11 & $2.145-11$ \\
\hline 20 & 477 & 2.035-05 & $1.481-05$ & $1.066-05$ & $6.186-06$ & $2.755-06$ & $1.522-06$ & $9.476-07$ & $6.375-07$ & $2.877-07$ & $1.519-07$ \\
\hline 20 & 478 & $2.749-05$ & $2.920-05$ & $3.179-05$ & $3.701-05$ & $4.470-05$ & 5.046-05 & $5.462-05$ & $5.761-05$ & 6.191-05 & $6.360-05$ \\
\hline 20 & 479 & $8.176-06$ & $5.684-06$ & $3.891-06$ & $2.095-06$ & $8.501-07$ & $4.471-07$ & $2.687-07$ & $1.760-07$ & 7.719-08 & $4.064-08$ \\
\hline 20 & 480 & 8.948-06 & $6.519-06$ & 4.891-06 & $3.438-06$ & $2.721-06$ & 2.669-06 & $2.717-06$ & $2.779-06$ & 2.911-06 & 2.996-06 \\
\hline 20 & 481 & $2.276-06$ & $1.832-06$ & $1.441-06$ & $9.673-07$ & $4.942-07$ & $2.906-07$ & $1.870-07$ & $1.285-07$ & $6.204-08$ & $3.509-08$ \\
\hline 20 & 482 & $4.760-06$ & $4.530-06$ & $4.531-06$ & $4.781-06$ & $5.315-06$ & $5.566-06$ & $5.699-06$ & $5.772-06$ & 5.993-06 & $6.112-06$ \\
\hline 20 & 483 & $5.102-06$ & $3.484-06$ & $2.344-06$ & $1.220-06$ & 4.791-07 & $2.398-07$ & $1.369-07$ & $8.530-08$ & $3.257-08$ & $1.532-08$ \\
\hline 20 & 484 & $9.335-07$ & $5.707-07$ & $3.415-07$ & $1.490-07$ & $4.857-08$ & $2.244-08$ & $1.226-08$ & 7.479-09 & 2.918-09 & $1.466-09$ \\
\hline 20 & 485 & $1.132-06$ & 7.193-07 & 4.579-07 & $2.417-07$ & $1.445-07$ & $1.187-07$ & 5.894-08 & $4.543-08$ & $9.777-08$ & 8.779-08 \\
\hline 20 & 486 & $2.992-05$ & $2.286-05$ & $1.734-05$ & $1.102-05$ & $5.359-06$ & $3.129-06$ & $2.028-06$ & $1.412-06$ & 7.034-07 & $4.108-07$ \\
\hline 20 & 487 & $1.538-05$ & $1.157-05$ & $8.630-06$ & $5.323-06$ & $2.448-06$ & $1.370-06$ & $8.571-07$ & 5.803-07 & $2.781-07$ & $1.618-07$ \\
\hline 20 & 488 & 2.013-05 & $1.504-05$ & $1.111-05$ & $6.776-06$ & $3.113-06$ & $1.791-06$ & $1.193-06$ & 8.835-07 & $5.667-07$ & $4.618-07$ \\
\hline 20 & 489 & 2.894-05 & $2.249-05$ & $1.743-05$ & $1.147-05$ & $5.850-06$ & $3.536-06$ & $2.350-06$ & $1.668-06$ & $8.587-07$ & $5.158-07$ \\
\hline 20 & 490 & $2.528-05$ & $2.087-05$ & $1.754-05$ & $1.375-05$ & $1.048-05$ & $9.458-06$ & $9.148-06$ & $9.137-06$ & $9.558-06$ & $1.011-05$ \\
\hline 20 & 491 & $9.180-06$ & 7.244-06 & $5.712-06$ & $3.862-06$ & $2.047-06$ & $1.269-06$ & $8.586-07$ & $6.173-07$ & $3.234-07$ & $1.954-07$ \\
\hline 20 & 492 & $1.212-05$ & $9.913-06$ & $8.533-06$ & $7.840-06$ & $8.705-06$ & $9.906-06$ & $1.093-05$ & $1.176-05$ & $1.334-05$ & $1.463-05$ \\
\hline 20 & 493 & $3.515-04$ & 3.866-04 & $4.288-04$ & $4.985-04$ & 5.990-04 & $6.727-04$ & 7.309-04 & $7.782-04$ & 8.692-04 & $9.407-04$ \\
\hline 20 & 494 & $5.093-08$ & $2.700-08$ & $1.375-08$ & $4.758-09$ & $1.149-09$ & $4.493-10$ & $2.114-10$ & $1.101-10$ & 2.959-11 & $1.330-11$ \\
\hline 20 & 497 & $9.581-08$ & $5.265-08$ & $2.768-08$ & $9.922-09$ & 2.357-09 & $8.092-10$ & $3.249-10$ & $1.474-10$ & $2.636-11$ & $1.349-11$ \\
\hline 20 & 498 & $9.110-06$ & $6.289-06$ & $4.271-06$ & $2.259-06$ & $8.870-07$ & $4.585-07$ & $2.755-07$ & $1.823-07$ & $8.210-08$ & $4.378-08$ \\
\hline 20 & 500 & 7.921-08 & $4.542-08$ & $2.474-08$ & $9.503-09$ & 2.155-09 & $8.013-10$ & $3.701-10$ & $1.864-10$ & 7.244-11 & $3.327-11$ \\
\hline 20 & 501 & $1.503-07$ & $9.089-08$ & $5.358-08$ & $2.355-08$ & 7.409-09 & $3.242-09$ & 1.697-09 & $1.016-09$ & $4.016-10$ & $2.123-10$ \\
\hline 20 & 503 & $9.425-06$ & $6.456-06$ & 4.399-06 & $2.446-06$ & $1.259-06$ & $9.696-07$ & $8.745-07$ & $8.372-07$ & $8.166-07$ & $8.295-07$ \\
\hline 20 & 504 & $2.156-05$ & $1.570-05$ & $1.131-05$ & $6.576-06$ & 2.938-06 & $1.629-06$ & $1.018-06$ & $6.877-07$ & $3.134-07$ & $1.671-07$ \\
\hline 20 & 505 & 2.197-05 & $1.920-05$ & $1.754-05$ & $1.662-05$ & $1.712-05$ & $1.832-05$ & $1.939-05$ & $2.022-05$ & $2.146-05$ & 2.194-05 \\
\hline 20 & 506 & $1.741-05$ & $1.292-05$ & $9.499-06$ & 5.691-06 & 2.637-06 & $1.487-06$ & $9.373-07$ & $6.350-07$ & $2.895-07$ & $1.543-07$ \\
\hline 20 & 507 & $1.422-05$ & $9.984-06$ & $6.905-06$ & $3.795-06$ & $1.592-06$ & $8.520-07$ & $5.145-07$ & $3.361-07$ & $1.454-07$ & 7.623-08 \\
\hline 20 & 508 & 5.204-05 & $5.782-05$ & 6.494-05 & $7.781-05$ & $9.561-05$ & $1.085-04$ & $1.178-04$ & $1.244-04$ & $1.339-04$ & $1.375-04$ \\
\hline 20 & 509 & $9.654-06$ & 7.554-06 & $6.274-06$ & $5.350-06$ & $5.308-06$ & $5.674-06$ & 5.986-06 & $6.234-06$ & $6.657-06$ & 6.894-06 \\
\hline 20 & 510 & $4.018-06$ & $3.625-06$ & $3.477-06$ & $3.520-06$ & $3.824-06$ & $3.986-06$ & $4.074-06$ & 4.124-06 & $4.263-06$ & 4.331-06 \\
\hline 20 & 511 & $4.159-06$ & $2.775-06$ & $1.820-06$ & $9.039-07$ & $3.332-07$ & $1.595-07$ & $8.737-08$ & $5.238-08$ & $1.824-08$ & 7.877-09 \\
\hline 20 & 512 & 4.691-06 & $3.191-06$ & $2.131-06$ & $1.088-06$ & $4.139-07$ & $1.999-07$ & $1.098-07$ & $6.577-08$ & $2.285-08$ & $9.940-09$ \\
\hline 20 & 513 & $8.222-06$ & $7.710-06$ & $7.587-06$ & $7.832-06$ & $8.584-06$ & $8.957-06$ & $9.158-06$ & $9.269-06$ & $9.591-06$ & $9.766-06$ \\
\hline 20 & 514 & $1.015-06$ & $6.123-07$ & 3.609-07 & $1.514-07$ & $4.503-08$ & $2.023-08$ & $1.110-08$ & 6.873-09 & 2.723-09 & $1.309-09$ \\
\hline 20 & 515 & $1.229-06$ & $7.565-07$ & $4.620-07$ & $2.212-07$ & $1.083-07$ & $8.940-08$ & $8.606-08$ & $7.826-08$ & $5.698-08$ & $6.402-08$ \\
\hline 20 & 516 & $1.351-06$ & $9.045-07$ & $6.176-07$ & $3.774-07$ & $2.802-07$ & $2.709-07$ & $2.792-07$ & $2.616-07$ & $1.913-07$ & $2.136-07$ \\
\hline 20 & 517 & $1.633-06$ & $1.022-06$ & $6.296-07$ & $2.917-07$ & $1.044-07$ & 4.974-08 & 2.719-08 & $1.644-08$ & 6.355-09 & 3.223-09 \\
\hline 20 & 518 & $5.011-07$ & $4.080-07$ & $3.264-07$ & $2.229-07$ & $1.247-07$ & $7.853-08$ & $5.327-08$ & $3.790-08$ & $1.834-08$ & $1.009-08$ \\
\hline 20 & 519 & 7.619-06 & $9.353-06$ & $1.145-05$ & $1.523-05$ & $2.179-05$ & $2.747-05$ & $3.248-05$ & $3.706-05$ & 4.677-05 & $5.422-05$ \\
\hline 20 & 520 & $1.988-06$ & $1.554-06$ & $1.196-06$ & 7.697-07 & $3.947-07$ & $2.357-07$ & $1.539-07$ & $1.065-07$ & $4.978-08$ & $2.705-08$ \\
\hline 20 & 521 & $1.102-04$ & 8.915-05 & 7.107-05 & $4.843-05$ & $2.623-05$ & $1.631-05$ & $1.108-05$ & 7.985-06 & $4.152-06$ & $2.481-06$ \\
\hline 20 & 522 & $1.887-04$ & $1.532-04$ & $1.225-04$ & $8.431-05$ & $4.656-05$ & 2.991-05 & $2.124-05$ & $1.608-05$ & $9.624-06$ & $6.902-06$ \\
\hline 20 & 523 & 2.695-04 & $2.183-04$ & $1.742-04$ & $1.189-04$ & $6.460-05$ & $4.025-05$ & $2.737-05$ & $1.975-05$ & $1.028-05$ & $6.145-06$ \\
\hline 20 & 524 & 7.383-07 & $5.480-07$ & $4.036-07$ & $2.481-07$ & $1.290-07$ & $9.294-08$ & $8.083-08$ & 7.444-08 & $6.575-08$ & $6.682-08$ \\
\hline
\end{tabular}


Table VII-continued ...

\begin{tabular}{|c|c|c|c|c|c|c|c|c|c|c|c|}
\hline \multirow[t]{2}{*}{$\overline{\mathrm{i}}$} & \multirow[t]{2}{*}{$\mathrm{j}$} & \multicolumn{10}{|c|}{$\begin{array}{c}\text { Collision strength } \Omega \\
\text { Scattered electron energy }(\mathrm{eV})\end{array}$} \\
\hline & & 10 & 50 & 100 & 200 & 400 & 600 & 800 & 1000 & 1500 & 2000 \\
\hline 20 & 525 & $6.311-07$ & $5.302-07$ & $4.368-07$ & $3.098-07$ & $1.797-07$ & $1.152-07$ & $7.890-08$ & $5.652-08$ & $2.788-08$ & $1.571-08$ \\
\hline 20 & 526 & $3.770-06$ & $3.978-06$ & $4.289-06$ & $4.940-06$ & $6.296-06$ & 7.643-06 & $8.922-06$ & $1.014-05$ & $1.283-05$ & $1.499-05$ \\
\hline 20 & 527 & $3.972-07$ & $3.216-07$ & $2.595-07$ & $1.814-07$ & $1.010-07$ & 6.395-08 & $4.410-08$ & $3.222-08$ & $1.721-08$ & $1.054-08$ \\
\hline 20 & 528 & $9.198-04$ & $1.040-03$ & $1.169-03$ & $1.382-03$ & $1.675-03$ & $1.876-03$ & $2.017-03$ & 2.119-03 & 2.303-03 & $2.428-03$ \\
\hline 20 & 529 & $1.568-07$ & $1.409-07$ & $1.301-07$ & $1.187-07$ & $1.064-07$ & $9.933-08$ & $9.476-08$ & $9.238-08$ & $9.197-08$ & $9.418-08$ \\
\hline 20 & 530 & $8.255-07$ & $6.319-07$ & 4.769-07 & $2.985-07$ & $1.458-07$ & $8.468-08$ & $5.435-08$ & $3.725-08$ & $1.730-08$ & $9.450-09$ \\
\hline 20 & 531 & $1.063-04$ & 7.761-05 & 5.553-05 & $3.175-05$ & $1.371-05$ & $7.152-06$ & 4.151-06 & 2.593-06 & $9.932-07$ & $4.666-07$ \\
\hline 20 & 532 & $1.481-04$ & $1.086-04$ & 7.843-05 & $4.504-05$ & $1.968-05$ & $1.049-05$ & $6.348-06$ & $4.204-06$ & $1.969-06$ & $1.258-06$ \\
\hline 20 & 533 & $1.920-04$ & $1.403-04$ & $1.004-04$ & $5.746-05$ & $2.484-05$ & $1.296-05$ & 7.527-06 & $4.705-06$ & 1.804-06 & $8.481-07$ \\
\hline 20 & 534 & $3.234-04$ & 3.931-04 & $4.572-04$ & $5.460-04$ & $6.561-04$ & 7.076-04 & 7.321-04 & 7.439-04 & 7.641-04 & 7.737-04 \\
\hline 20 & 535 & $9.591-06$ & $8.595-06$ & 7.908-06 & $7.146-06$ & $6.574-06$ & $6.402-06$ & $6.372-06$ & $6.455-06$ & 6.633-06 & $6.630-06$ \\
\hline 20 & 536 & $2.364-05$ & $2.654-05$ & $2.970-05$ & $3.493-05$ & $4.222-05$ & $4.722-05$ & $5.072-05$ & $5.327-05$ & $5.790-05$ & $6.101-05$ \\
\hline 20 & 537 & $1.902-06$ & $1.502-06$ & $1.169-06$ & $7.652-07$ & $3.915-07$ & $2.343-07$ & $1.550-07$ & $1.098-07$ & 5.604-08 & $3.343-08$ \\
\hline 20 & 538 & $3.739-06$ & $3.082-06$ & $2.504-06$ & $1.762-06$ & $9.961-07$ & $6.364-07$ & $4.400-07$ & $3.210-07$ & $1.695-07$ & $1.019-07$ \\
\hline 20 & 539 & $9.951-08$ & $6.751-08$ & $4.652-08$ & $2.866-08$ & $2.191-08$ & $2.149-08$ & $2.166-08$ & $2.065-08$ & $2.131-08$ & $3.116-08$ \\
\hline 20 & 540 & $1.033-05$ & $9.708-06$ & $9.249-06$ & $8.821-06$ & 8.629-06 & 8.789-06 & 8.998-06 & $9.195-06$ & $9.660-06$ & $1.003-05$ \\
\hline 20 & 541 & $3.532-06$ & $2.956-06$ & $2.442-06$ & $1.764-06$ & $1.036-06$ & $6.784-07$ & $4.770-07$ & $3.520-07$ & $1.891-07$ & $1.148-07$ \\
\hline 20 & 542 & $2.476-06$ & $2.139-06$ & $1.829-06$ & $1.399-06$ & 8.794-07 & $6.001-07$ & $4.335-07$ & $3.262-07$ & $1.815-07$ & $1.130-07$ \\
\hline 20 & 543 & 8.036-07 & $6.983-07$ & $6.037-07$ & $4.738-07$ & $3.228-07$ & $2.500-07$ & $2.119-07$ & $1.909-07$ & $1.655-07$ & $1.513-07$ \\
\hline 20 & 544 & $4.869-07$ & 4.108-07 & $3.426-07$ & $2.499-07$ & $1.430-07$ & $9.037-08$ & $6.106-08$ & $4.342-08$ & $2.196-08$ & $1.298-08$ \\
\hline 20 & 545 & $7.305-07$ & 7.313-07 & $7.547-07$ & $8.361-07$ & $1.018-06$ & $1.185-06$ & $1.322-06$ & $1.436-06$ & $1.645-06$ & $1.786-06$ \\
\hline 20 & 546 & $1.011-06$ & $8.112-07$ & $6.434-07$ & $4.380-07$ & $2.400-07$ & $1.516-07$ & $1.044-07$ & 7.613-08 & $4.021-08$ & $2.414-08$ \\
\hline 20 & 547 & $1.770-06$ & $1.307-06$ & $9.456-07$ & $5.510-07$ & $2.436-07$ & $1.297-07$ & 7.675-08 & $4.879-08$ & $1.937-08$ & 9.359-09 \\
\hline 20 & 548 & $3.811-06$ & 4.127-06 & $4.464-06$ & 4.998-06 & $5.775-06$ & $6.176-06$ & $6.374-06$ & $6.475-06$ & $6.631-06$ & $6.682-06$ \\
\hline 20 & 549 & $3.716-06$ & $3.907-06$ & $4.141-06$ & $4.545-06$ & $5.192-06$ & $5.516-06$ & $5.670-06$ & $5.743-06$ & $5.872-06$ & $5.928-06$ \\
\hline 20 & 550 & $2.932-06$ & $2.125-06$ & $1.508-06$ & $8.512-07$ & $3.623-07$ & $1.869-07$ & $1.074-07$ & $6.652-08$ & $2.491-08$ & $1.148-08$ \\
\hline 20 & 551 & $3.021-07$ & $3.708-07$ & $4.670-07$ & $6.541-07$ & $9.471-07$ & $1.169-06$ & $1.324-06$ & $1.445-06$ & $1.666-06$ & $1.818-06$ \\
\hline 20 & 552 & $1.021-07$ & 7.789-08 & $5.790-08$ & $3.453-08$ & $1.491-08$ & 7.765-09 & 4.538-09 & 2.889-09 & $1.228-09$ & $6.581-10$ \\
\hline 20 & 553 & $3.951-08$ & $2.723-08$ & $1.937-08$ & $1.476-08$ & $1.417-08$ & $1.466-08$ & $1.483-08$ & $1.479-08$ & $1.415-08$ & $1.450-08$ \\
\hline 20 & 554 & $7.549-08$ & $5.173-08$ & $3.489-08$ & $1.841-08$ & 7.072-09 & 3.467-09 & 1.933-09 & $1.170-09$ & $4.253-10$ & $1.908-10$ \\
\hline 20 & 555 & $2.076-05$ & $2.417-05$ & $2.781-05$ & $3.381-05$ & $4.200-05$ & $4.757-05$ & $5.152-05$ & $5.446-05$ & 5.907-05 & $6.127-05$ \\
\hline 20 & 556 & 5.847-07 & $4.480-07$ & 3.361-07 & $2.071-07$ & $9.699-08$ & $5.386-08$ & 3.309-08 & $2.179-08$ & $9.362-09$ & 4.801-09 \\
\hline 20 & 557 & $1.033-06$ & $1.110-06$ & $1.188-06$ & $1.304-06$ & $1.470-06$ & $1.555-06$ & $1.592-06$ & $1.607-06$ & $1.660-06$ & $1.708-06$ \\
\hline 20 & 558 & $1.875-07$ & $1.281-07$ & 8.633-08 & $4.489-08$ & $1.704-08$ & 8.204-09 & 4.607-09 & 2.925-09 & $1.343-09$ & $8.035-10$ \\
\hline 20 & 559 & 3.339-07 & $2.441-07$ & $1.814-07$ & $1.203-07$ & 8.871-08 & 8.094-08 & $8.416-08$ & 8.733-08 & 9.987-08 & $1.143-07$ \\
\hline
\end{tabular}


TABLE VIII:

\begin{tabular}{|c|c|c|c|c|c|c|c|c|c|c|c|}
\hline \multirow[t]{2}{*}{ i } & \multirow[t]{2}{*}{$\mathrm{j}$} & \multicolumn{10}{|c|}{$\begin{array}{l}\text { Collision strength } \Omega \\
\text { Scattered electron energy }\end{array}$} \\
\hline & & 10 & 50 & 100 & 200 & 400 & 600 & 800 & 1000 & 1500 & 2000 \\
\hline 1 & 2 & $6.724-02$ & $2.139-01$ & $2.335-01$ & $1.958-01$ & $1.253-01$ & $9.269-02$ & $7.719-02$ & $6.914-02$ & $6.121-02$ & $5.893-02$ \\
\hline 1 & 3 & $1.650-02$ & $1.489-02$ & $1.309-02$ & $1.025-02$ & 6.759-03 & $4.859-03$ & $3.722-03$ & 2.995-03 & $2.027-03$ & $1.577-03$ \\
\hline 1 & 4 & $2.315-02$ & $2.080-02$ & $1.819-02$ & $1.406-02$ & 8.981-03 & $6.205-03$ & $4.535-03$ & $3.459-03$ & 2.009-03 & $1.318-03$ \\
\hline 1 & 5 & $1.388-02$ & $1.247-02$ & $1.090-02$ & $8.420-03$ & 5.373-03 & $3.707-03$ & $2.705-03$ & 2.059-03 & $1.188-03$ & 7.726-04 \\
\hline 1 & 6 & $2.564-02$ & $2.300-02$ & $2.007-02$ & $1.555-02$ & $9.980-03$ & 6.911-03 & 5.056-03 & 3.859-03 & $2.266-03$ & 1.501-03 \\
\hline 1 & 7 & $6.374-01$ & $6.883-01$ & 7.419-01 & $8.268-01$ & $9.505-01$ & $1.038+00$ & $1.104+00$ & $1.158+00$ & $1.261+00$ & $1.337+00$ \\
\hline 1 & 8 & $3.672-01$ & 3.939-01 & $4.229-01$ & $4.696-01$ & 5.396-01 & 5.904-01 & $6.296-01$ & 6.616-01 & 7.231-01 & 7.686-01 \\
\hline 1 & 9 & $5.617-01$ & $6.022-01$ & $6.466-01$ & $7.183-01$ & 8.268-01 & $9.057-01$ & $9.670-01$ & $1.017+00$ & $1.113+00$ & $1.184+00$ \\
\hline 1 & 10 & $3.263-01$ & 3.491-01 & $3.742-01$ & $4.146-01$ & $4.760-01$ & 5.209-01 & $5.558-01$ & 5.844-01 & $6.392-01$ & 6.800-01 \\
\hline 1 & 11 & $1.119-03$ & $9.389-04$ & $7.585-04$ & $5.190-04$ & 2.876-04 & $1.749-04$ & $1.158-04$ & 8.179-05 & 4.093-05 & 2.499-05 \\
\hline 1 & 12 & $3.740-03$ & 3.996-03 & $4.259-03$ & $4.525-03$ & 8.712-03 & $6.551-03$ & $6.177-03$ & $6.325-03$ & $5.902-03$ & 6.069-03 \\
\hline 1 & 13 & $3.125-03$ & $3.141-03$ & $3.162-03$ & $3.138-03$ & 5.484-03 & 4.094-03 & 3.823-03 & 3.886-03 & $3.600-03$ & $3.688-03$ \\
\hline 1 & 14 & 2.932-03 & $2.881-03$ & 2.811-03 & $2.702-03$ & 2.599-03 & $2.543-03$ & $2.507-03$ & 2.481-03 & $2.443-03$ & 2.431-03 \\
\hline 1 & 15 & $1.866-03$ & $1.904-03$ & $1.947-03$ & $2.034-03$ & $2.235-03$ & $2.932-03$ & $2.644-03$ & $2.375-03$ & $2.463-03$ & 2.427-03 \\
\hline 1 & 16 & $5.196-03$ & $5.357-03$ & $5.696-03$ & $6.615-03$ & 8.720-03 & $1.068-02$ & $1.260-02$ & $1.430-02$ & $1.780-02$ & 2.066-02 \\
\hline 1 & 17 & $4.612-02$ & $4.606-02$ & $4.585-02$ & $4.552-02$ & 4.545-02 & $4.548-02$ & $4.549-02$ & $4.550-02$ & 4.551-02 & 4.551-02 \\
\hline 1 & 18 & $2.241-02$ & $2.066-02$ & $1.893-02$ & 1.684-02 & $1.518-02$ & $1.473-02$ & $1.477-02$ & $1.537-02$ & $1.673-02$ & $1.647-02$ \\
\hline 1 & 19 & $1.736-01$ & $1.905-01$ & $2.111-01$ & $2.505-01$ & $3.177-01$ & $3.727-01$ & $4.189-01$ & 4.598-01 & $5.442-01$ & 6.089-01 \\
\hline 1 & 20 & $3.759-02$ & $3.216-02$ & $2.722-02$ & 2.119-02 & $1.599-02$ & $1.398-02$ & $1.307-02$ & $1.318-02$ & $1.408-02$ & $1.345-02$ \\
\hline 1 & 21 & $3.453-03$ & 2.926-03 & $2.402-03$ & $1.704-03$ & $1.015-03$ & 6.908-04 & $5.108-04$ & 3.998-04 & $2.540-04$ & $1.855-04$ \\
\hline 1 & 22 & $4.372-03$ & $3.690-03$ & $3.015-03$ & $2.115-03$ & $1.226-03$ & 8.079-04 & $5.759-04$ & $4.330-04$ & $2.455-04$ & $1.571-04$ \\
\hline 1 & 23 & $7.765-05$ & $6.517-05$ & 5.326-05 & $3.744-05$ & 2.138-05 & $1.379-05$ & $9.598-06$ & 7.039-06 & $3.776-06$ & 2.310-06 \\
\hline 1 & 24 & $1.051-01$ & $1.092-01$ & $1.131-01$ & $1.186-01$ & $1.256-01$ & $1.294-01$ & $1.318-01$ & $1.333-01$ & $1.356-01$ & $1.368-01$ \\
\hline 1 & 25 & 6.921-04 & $5.890-04$ & 4.894-04 & $3.609-04$ & $2.421-04$ & $1.911-04$ & $1.669-04$ & $1.632-04$ & $1.692-04$ & $1.510-04$ \\
\hline 1 & 26 & $4.581-03$ & $5.154-03$ & $5.950-03$ & 7.613-03 & $1.081-02$ & $1.357-02$ & $1.605-02$ & $1.831-02$ & 2.299-02 & 2.661-02 \\
\hline 1 & 27 & 4.337-03 & $4.270-03$ & $4.322-03$ & $4.650-03$ & $5.620-03$ & $6.641-03$ & 7.632-03 & $8.577-03$ & $1.060-02$ & $1.220-02$ \\
\hline 1 & 28 & $4.557-03$ & $5.674-03$ & $7.101-03$ & $9.890-03$ & $1.496-02$ & $1.917-02$ & $2.289-02$ & $2.625-02$ & $3.314-02$ & 3.845-02 \\
\hline 1 & 29 & $3.780-03$ & 4.391-03 & $5.210-03$ & $6.875-03$ & $1.001-02$ & $1.268-02$ & $1.506-02$ & $1.723-02$ & $2.170-02$ & 2.514-02 \\
\hline 1 & 30 & 2.979-03 & $2.548-03$ & $2.140-03$ & $1.569-03$ & 9.304-04 & $6.102-04$ & $4.275-04$ & 3.141-04 & $1.673-04$ & 9.995-05 \\
\hline 1 & 31 & $9.525-06$ & 7.587-06 & $5.866-06$ & $3.759-06$ & $1.865-06$ & $1.080-06$ & $6.841-07$ & 4.604-07 & 2.039-07 & $1.060-07$ \\
\hline 1 & 32 & $1.669-03$ & $1.462-03$ & $1.273-03$ & $1.021-03$ & 7.721-04 & $6.778-04$ & $6.478-04$ & $6.484-04$ & 6.961-04 & 7.573-04 \\
\hline 1 & 33 & $1.439-03$ & $1.240-03$ & $1.051-03$ & 7.887-04 & 4.947-04 & $3.544-04$ & $2.761-04$ & 2.304-04 & $1.803-04$ & 1.636-04 \\
\hline 1 & 34 & $1.579-03$ & $1.362-03$ & $1.156-03$ & $8.682-04$ & 5.497-04 & 3.949-04 & $3.111-04$ & 2.627-04 & 2.091-04 & 1.924-04 \\
\hline 1 & 35 & $1.128-03$ & $9.690-04$ & $8.174-04$ & $6.025-04$ & $3.595-04$ & $2.367-04$ & $1.663-04$ & $1.224-04$ & $6.535-05$ & 3.918-05 \\
\hline 1 & 36 & $1.473-02$ & $1.914-02$ & $2.477-02$ & $3.574-02$ & 5.574-02 & 7.239-02 & 8.709-02 & $1.004-01$ & $1.278-01$ & $1.488-01$ \\
\hline 1 & 37 & 2.974-04 & 2.594-04 & $2.234-04$ & $1.727-04$ & $1.168-04$ & 8.944-05 & 7.395-05 & $6.435-05$ & $5.247-05$ & 4.797-05 \\
\hline 1 & 38 & 8.201-03 & 6.919-03 & $5.685-03$ & $4.042-03$ & 2.373-03 & $1.593-03$ & $1.166-03$ & $9.096-04$ & $5.902-04$ & 4.497-04 \\
\hline 1 & 39 & $9.742-03$ & $8.204-03$ & $6.715-03$ & $4.725-03$ & 2.698-03 & $1.755-03$ & $1.231-03$ & $9.126-04$ & $5.148-04$ & 3.393-04 \\
\hline 1 & 40 & $7.842-03$ & $6.592-03$ & $5.389-03$ & $3.785-03$ & $2.150-03$ & $1.381-03$ & $9.574-04$ & 7.011-04 & $3.785-04$ & 2.340-04 \\
\hline 1 & 41 & $2.486-03$ & $3.263-03$ & $4.262-03$ & $6.231-03$ & 9.863-03 & $1.291-02$ & $1.561-02$ & $1.808-02$ & 2.316-02 & 2.706-02 \\
\hline 1 & 42 & $5.665-06$ & $4.513-06$ & $3.514-06$ & $2.290-06$ & $1.188-06$ & $7.250-07$ & $4.845-07$ & $3.433-07$ & $1.702-07$ & $9.718-08$ \\
\hline 1 & 43 & $2.556-03$ & $2.141-03$ & $1.744-03$ & $1.219-03$ & 6.884-04 & $4.417-04$ & $3.071-04$ & $2.262-04$ & $1.251-04$ & 8.027-05 \\
\hline 1 & 44 & $5.405-03$ & 4.559-03 & $3.746-03$ & 2.671-03 & $1.597-03$ & $1.108-03$ & 8.428-04 & $6.847-04$ & 4.944-04 & 4.161-04 \\
\hline 1 & 45 & $9.072-03$ & $7.778-03$ & $6.550-03$ & 4.954-03 & $3.405-03$ & $2.722-03$ & $2.376-03$ & $2.184-03$ & $1.977-03$ & 1.907-03 \\
\hline 1 & 46 & $3.164-03$ & $2.647-03$ & $2.153-03$ & $1.499-03$ & 8.408-04 & $5.352-04$ & $3.686-04$ & 2.686-04 & $1.437-04$ & $8.822-05$ \\
\hline 1 & 47 & $1.781-02$ & $2.003-02$ & $2.248-02$ & $2.662-02$ & $3.266-02$ & $3.658-02$ & $3.952-02$ & $4.175-02$ & $4.549-02$ & 4.780-02 \\
\hline 1 & 48 & $1.234-02$ & $1.330-02$ & $1.441-02$ & $1.639-02$ & $1.947-02$ & $2.154-02$ & 2.314-02 & $2.437-02$ & $2.645-02$ & 2.774-02 \\
\hline 1 & 49 & $4.132-03$ & $4.167-03$ & $4.245-03$ & $4.468-03$ & 4.949-03 & $5.325-03$ & $5.640-03$ & 5.893-03 & $6.340-03$ & $6.630-03$ \\
\hline 1 & 50 & $1.027-03$ & $9.324-04$ & $8.450-04$ & 7.406-04 & 6.603-04 & $6.386-04$ & $6.372-04$ & $6.425-04$ & $6.620-04$ & 6.792-04 \\
\hline 1 & 51 & $1.304-04$ & $1.131-04$ & $9.652-05$ & $7.472-05$ & 5.315-05 & $4.335-05$ & 3.809-05 & $3.498-05$ & 3.121-05 & 2.961-05 \\
\hline 1 & 52 & 2.043-02 & $2.128-02$ & $2.209-02$ & 2.328-02 & $2.481-02$ & $2.570-02$ & 2.626-02 & 2.664-02 & $2.720-02$ & 2.749-02 \\
\hline 1 & 53 & $2.272-03$ & $2.061-03$ & $1.859-03$ & $1.614-03$ & $1.435-03$ & $1.381-03$ & $1.373-03$ & $1.382-03$ & $1.423-03$ & $1.457-03$ \\
\hline 1 & 54 & 4.179-02 & 4.739-02 & 5.355-02 & $6.388-02$ & 7.898-02 & 8.873-02 & $9.601-02$ & $1.016-01$ & 1.109-01 & 1.167-01 \\
\hline 1 & 55 & $1.050-03$ & 8.801-04 & 7.177-04 & $5.030-04$ & 2.860-04 & $1.847-04$ & $1.293-04$ & $9.596-05$ & $5.446-05$ & $3.607-05$ \\
\hline 1 & 56 & $1.521-02$ & $1.712-02$ & $1.924-02$ & $2.282-02$ & 2.811-02 & $3.155-02$ & $3.412-02$ & 3.609-02 & $3.942-02$ & 4.145-02 \\
\hline 1 & 57 & $5.935-04$ & $5.501-04$ & 5.099-04 & $4.613-04$ & $4.217-04$ & $4.090-04$ & $4.040-04$ & $4.023-04$ & $4.028-04$ & 4.044-04 \\
\hline 1 & 58 & $1.285-03$ & $1.583-03$ & $1.982-03$ & $2.803-03$ & $4.387-03$ & $5.757-03$ & 6.984-03 & 8.116-03 & $1.049-02$ & $1.232-02$ \\
\hline 1 & 59 & $1.227-03$ & $1.095-03$ & $9.731-04$ & $8.122-04$ & $6.550-04$ & $6.047-04$ & $5.948-04$ & $6.056-04$ & $6.650-04$ & 7.299-04 \\
\hline 1 & 60 & $1.997-03$ & $1.755-03$ & $1.512-03$ & $1.151-03$ & 7.232-04 & 4.964-04 & $3.633-04$ & $2.790-04$ & $1.699-04$ & $1.225-04$ \\
\hline
\end{tabular}


Table VIII-continued ...

\begin{tabular}{|c|c|c|c|c|c|c|c|c|c|c|c|}
\hline \multirow[t]{2}{*}{$\overline{\mathrm{i}}$} & \multirow[t]{2}{*}{$\bar{j}$} & \multicolumn{10}{|c|}{$\begin{array}{c}\text { Collision strength } \Omega \\
\text { Scattered electron energy }(\mathrm{eV})\end{array}$} \\
\hline & & 10 & 50 & 100 & 200 & 400 & 600 & 800 & 1000 & 1500 & 2000 \\
\hline 1 & 61 & $1.395-03$ & $1.719-03$ & $2.168-03$ & $3.106-03$ & 4.981-03 & $6.609-03$ & $8.089-03$ & $9.459-03$ & $1.231-02$ & $1.450-02$ \\
\hline 1 & 62 & $1.415-03$ & $1.517-03$ & $1.661-03$ & $1.967-03$ & 2.561-03 & 3.083-03 & $3.546-03$ & $3.972-03$ & 4.869-03 & $5.558-03$ \\
\hline 1 & 63 & $3.633-05$ & $3.297-05$ & $2.980-05$ & $2.588-05$ & 2.304-05 & 2.294-05 & $2.390-05$ & $2.538-05$ & 2.946-05 & $3.308-05$ \\
\hline 1 & 64 & $4.346-05$ & $3.803-05$ & $3.252-05$ & $2.446-05$ & $1.519-05$ & $1.042-05$ & 7.668-06 & 5.961-06 & $3.802-06$ & 2.864-06 \\
\hline 1 & 65 & $2.707-05$ & $2.342-05$ & $1.976-05$ & $1.452-05$ & 8.690-06 & $5.747-06$ & 4.053-06 & $3.000-06$ & $1.644-06$ & $1.027-06$ \\
\hline 1 & 66 & $6.444-03$ & $5.383-03$ & $4.371-03$ & $3.044-03$ & 1.716-03 & $1.103-03$ & $7.720-04$ & $5.744-04$ & $3.312-04$ & $2.251-04$ \\
\hline 1 & 67 & $1.137-02$ & $1.233-02$ & $1.343-02$ & $1.541-02$ & 1.852-02 & 2.064-02 & $2.227-02$ & 2.354-02 & $2.574-02$ & $2.713-02$ \\
\hline 1 & 68 & 7.624-03 & 8.293-03 & $9.063-03$ & $1.043-02$ & $1.257-02$ & $1.401-02$ & $1.511-02$ & $1.597-02$ & $1.749-02$ & $1.844-02$ \\
\hline 1 & 69 & $6.917-03$ & 7.093-03 & $7.342-03$ & 7.899-03 & 8.971-03 & $9.773-03$ & $1.042-02$ & 1.094-02 & $1.190-02$ & $1.251-02$ \\
\hline 1 & 70 & 2.868-04 & $3.486-04$ & $4.333-04$ & $6.076-04$ & $9.545-04$ & $1.258-03$ & $1.530-03$ & $1.786-03$ & $2.325-03$ & $2.731-03$ \\
\hline 1 & 71 & $1.443-03$ & $1.253-03$ & $1.068-03$ & $8.227-04$ & 5.738-04 & $4.582-04$ & 3.944-04 & $3.562-04$ & $3.102-04$ & $2.861-04$ \\
\hline 1 & 72 & 5.930-03 & $6.283-03$ & $6.708-03$ & $7.505-03$ & 8.831-03 & $9.758-03$ & $1.048-02$ & $1.105-02$ & $1.205-02$ & $1.270-02$ \\
\hline 1 & 73 & $2.300-04$ & $2.585-04$ & $3.008-04$ & $3.922-04$ & 5.844-04 & 7.579-04 & $9.157-04$ & $1.066-03$ & $1.385-03$ & $1.626-03$ \\
\hline 1 & 74 & $4.314-05$ & $3.658-05$ & 2.996-05 & $2.087-05$ & $1.172-05$ & $7.445-06$ & $5.156-06$ & $3.823-06$ & $2.261-06$ & $1.639-06$ \\
\hline 1 & 75 & $1.212-04$ & $1.053-04$ & 8.881-05 & $6.525-05$ & 3.978-05 & 2.693-05 & $1.953-05$ & $1.493-05$ & 8.998-06 & $6.345-06$ \\
\hline 1 & 76 & $2.071-04$ & $1.805-04$ & $1.529-04$ & $1.136-04$ & 7.119-05 & $4.990-05$ & $3.779-05$ & $3.028-05$ & $2.064-05$ & $1.645-05$ \\
\hline 1 & 77 & $2.204-04$ & $1.916-04$ & $1.618-04$ & $1.190-04$ & $7.250-05$ & 4.889-05 & $3.525-05$ & 2.671-05 & $1.560-05$ & $1.058-05$ \\
\hline 1 & 78 & $6.179-05$ & $5.275-05$ & 4.394-05 & $3.172-05$ & $1.878-05$ & $1.255-05$ & $9.059-06$ & $6.928-06$ & $4.258-06$ & $3.082-06$ \\
\hline 1 & 79 & $9.021-05$ & $1.001-04$ & $1.158-04$ & $1.510-04$ & $2.293-04$ & $3.007-04$ & $3.660-04$ & $4.291-04$ & $5.627-04$ & $6.620-04$ \\
\hline 1 & 80 & $1.800-04$ & $1.568-04$ & $1.327-04$ & $9.805-05$ & 5.987-05 & $4.026-05$ & $2.880-05$ & $2.156-05$ & $1.205-05$ & 7.676-06 \\
\hline 1 & 81 & $6.525-05$ & $5.705-05$ & 4.839-05 & $3.583-05$ & $2.224-05$ & $1.522-05$ & $1.109-05$ & 8.466-06 & 4.941-06 & $3.261-06$ \\
\hline 1 & 82 & $1.304-04$ & $1.142-04$ & $9.722-05$ & $7.283-05$ & 4.670-05 & $3.349-05$ & $2.587-05$ & 2.111-05 & $1.492-05$ & $1.209-05$ \\
\hline 1 & 83 & $1.663-04$ & $1.447-04$ & $1.222-04$ & 8.993-05 & $5.476-05$ & $3.690-05$ & $2.658-05$ & 2.011-05 & $1.172-05$ & $7.972-06$ \\
\hline 1 & 84 & $1.231-04$ & $1.074-04$ & $9.168-05$ & $7.092-05$ & 5.133-05 & 4.392-05 & 4.071-05 & 3.906-05 & $3.932-05$ & $4.215-05$ \\
\hline 1 & 85 & $1.105-04$ & $1.003-04$ & $9.035-05$ & $7.746-05$ & 6.615-05 & $6.287-05$ & $6.180-05$ & $6.160-05$ & $6.352-05$ & 6.616-05 \\
\hline 1 & 86 & $9.164-05$ & 8.913-05 & $8.728-05$ & 8.671-05 & 9.116-05 & $9.722-05$ & $1.020-04$ & $1.060-04$ & $1.145-04$ & $1.204-04$ \\
\hline 1 & 87 & $4.018-04$ & $4.115-04$ & $4.200-04$ & 4.321-04 & 4.497-04 & 4.609-04 & 4.681-04 & $4.733-04$ & $4.815-04$ & 4.863-04 \\
\hline 1 & 88 & $6.443-05$ & $5.482-05$ & $4.504-05$ & $3.158-05$ & $1.810-05$ & $1.185-05$ & $8.518-06$ & 6.591-06 & $4.353-06$ & $3.476-06$ \\
\hline 1 & 89 & $1.988-04$ & $1.649-04$ & $1.328-04$ & $9.186-05$ & 5.382-05 & $3.802-05$ & $3.052-05$ & 2.671-05 & $2.345-05$ & $2.318-05$ \\
\hline 1 & 90 & $2.530-04$ & $2.082-04$ & $1.656-04$ & $1.107-04$ & $5.805-05$ & $3.482-05$ & $2.289-05$ & $1.610-05$ & $8.080-06$ & $4.713-06$ \\
\hline 1 & 91 & $2.644-04$ & $2.180-04$ & $1.739-04$ & $1.166-04$ & 6.146-05 & $3.708-05$ & $2.447-05$ & $1.722-05$ & $8.617-06$ & $5.028-06$ \\
\hline 1 & 92 & $2.021-04$ & $1.675-04$ & $1.343-04$ & $9.095-05$ & 4.863-05 & $2.970-05$ & $1.979-05$ & $1.402-05$ & 7.085-06 & 4.163-06 \\
\hline 1 & 93 & 7.895-04 & 8.504-04 & $9.230-04$ & $1.055-03$ & $1.270-03$ & $1.424-03$ & $1.537-03$ & $1.626-03$ & $1.784-03$ & $1.879-03$ \\
\hline 1 & 94 & $5.432-04$ & 4.679-04 & $3.923-04$ & $2.869-04$ & $1.740-04$ & $1.176-04$ & $8.520-05$ & $6.504-05$ & $3.910-05$ & $2.744-05$ \\
\hline 1 & 95 & $3.079-04$ & $2.808-04$ & $2.565-04$ & $2.312-04$ & $2.225-04$ & $2.318-04$ & $2.467-04$ & $2.633-04$ & 3.039-04 & 3.393-04 \\
\hline 1 & 96 & $\begin{array}{l}1.668-04 \\
\text { available c }\end{array}$ & $\begin{array}{l}1.489-04 \\
\text { request }\end{array}$ & $1.320-04$ & $1.119-04$ & 9.761-05 & $9.555-05$ & $9.806-05$ & $1.024-04$ & $1.153-04$ & $1.276-04$ \\
\hline 15 & 172 & $3.773-04$ & $3.951-04$ & 4.197-04 & $4.726-04$ & 5.729-04 & $6.616-04$ & 7.385-04 & 8.089-04 & $9.597-04$ & $1.077-03$ \\
\hline 15 & 173 & $4.751-04$ & $5.026-04$ & $5.365-04$ & $6.021-04$ & 7.212-04 & $8.171-04$ & 8.947-04 & $9.569-04$ & $1.066-03$ & $1.136-03$ \\
\hline 15 & 174 & $6.746-04$ & 7.399-04 & $8.143-04$ & $9.401-04$ & $1.131-03$ & $1.261-03$ & $1.359-03$ & $1.434-03$ & $1.559-03$ & $1.632-03$ \\
\hline 15 & 175 & $2.024-04$ & $1.604-04$ & $1.243-04$ & $8.086-05$ & $4.251-05$ & $2.715-05$ & $1.949-05$ & $1.520-05$ & $1.042-05$ & $8.628-06$ \\
\hline 15 & 176 & $2.390-04$ & $2.174-04$ & $2.002-04$ & $1.819-04$ & $1.725-04$ & $1.726-04$ & $1.737-04$ & $1.749-04$ & $1.773-04$ & $1.779-04$ \\
\hline 15 & 177 & $1.726-04$ & $1.326-04$ & $9.933-05$ & $6.071-05$ & $2.800-05$ & $1.535-05$ & $9.223-06$ & $5.912-06$ & $2.387-06$ & $1.157-06$ \\
\hline 15 & 178 & $1.929-04$ & $2.086-04$ & $2.269-04$ & $2.589-04$ & 3.096-04 & $3.463-04$ & $3.754-04$ & $3.983-04$ & $4.362-04$ & $4.568-04$ \\
\hline 15 & 179 & 3.369-04 & $3.462-04$ & 3.593-04 & $3.834-04$ & $4.264-04$ & $4.559-04$ & $4.743-04$ & $4.868-04$ & $5.036-04$ & 5.089-04 \\
\hline 15 & 180 & 3.821-04 & $3.719-04$ & $3.689-04$ & $3.802-04$ & $4.260-04$ & 4.771-04 & $5.245-04$ & 5.696-04 & $6.701-04$ & $7.508-04$ \\
\hline 15 & 181 & $2.819-04$ & $3.234-04$ & $3.651-04$ & $4.237-04$ & 5.024-04 & 5.466-04 & $5.675-04$ & 5.794-04 & $5.992-04$ & $6.078-04$ \\
\hline 15 & 182 & $1.329-04$ & $1.097-04$ & 8.966-05 & $6.577-05$ & 4.588-05 & $3.901-05$ & $3.636-05$ & $3.557-05$ & 3.664-05 & $3.867-05$ \\
\hline 15 & 183 & $6.186-05$ & $4.900-05$ & $3.775-05$ & $2.407-05$ & $1.183-05$ & $6.784-06$ & $4.269-06$ & 2.865-06 & $1.278-06$ & $6.779-07$ \\
\hline 15 & 184 & $2.043-03$ & 2.239-03 & $2.466-03$ & $2.882-03$ & $3.582-03$ & 4.163-03 & 4.651-03 & 5.091-03 & $6.036-03$ & $6.778-03$ \\
\hline 15 & 185 & $5.216-04$ & $5.661-04$ & $6.162-04$ & 7.019-04 & 8.430-04 & $9.537-04$ & $1.039-03$ & $1.114-03$ & $1.274-03$ & $1.396-03$ \\
\hline 15 & 186 & $7.322-05$ & 7.677-05 & $8.268-05$ & $9.622-05$ & $1.238-04$ & $1.498-04$ & $1.741-04$ & $1.975-04$ & 2.503-04 & 2.930-04 \\
\hline 15 & 187 & $1.094-04$ & $9.833-05$ & $8.730-05$ & $7.259-05$ & 5.970-05 & $5.520-05$ & $5.392-05$ & 5.399-05 & $5.550-05$ & 5.679-05 \\
\hline 15 & 188 & $1.496-03$ & $1.515-03$ & $1.529-03$ & $1.553-03$ & 1.599-03 & $1.634-03$ & $1.659-03$ & $1.679-03$ & $1.712-03$ & $1.731-03$ \\
\hline 15 & 189 & $3.978-04$ & $3.659-04$ & $3.395-04$ & $3.166-04$ & $3.170-04$ & 3.337-04 & $3.543-04$ & $3.752-04$ & $4.227-04$ & $4.622-04$ \\
\hline 15 & 190 & $6.528-05$ & $5.579-05$ & $4.623-05$ & $3.326-05$ & 2.043-05 & $1.466-05$ & $1.213-05$ & $1.099-05$ & $9.310-06$ & $9.132-06$ \\
\hline 15 & 191 & $1.518-03$ & $1.581-03$ & $1.665-03$ & $1.844-03$ & 2.174-03 & $2.448-03$ & $2.685-03$ & 2.896-03 & $3.332-03$ & $3.675-03$ \\
\hline 15 & 192 & $8.667-04$ & $8.776-04$ & 8.868-04 & $9.025-04$ & 9.326-04 & $9.547-04$ & $9.712-04$ & $9.837-04$ & $1.003-03$ & $1.016-03$ \\
\hline 15 & 193 & $3.567-05$ & $2.781-05$ & 2.183-05 & $1.577-05$ & $1.173-05$ & $1.062-05$ & $1.020-05$ & 1.004-05 & 9.946-06 & 9.955-06 \\
\hline
\end{tabular}


Table VIII-continued ...

\begin{tabular}{|c|c|c|c|c|c|c|c|c|c|c|c|}
\hline \multirow[t]{2}{*}{$\overline{\mathrm{i}}$} & \multirow[t]{2}{*}{$\mathrm{j}$} & \multicolumn{10}{|c|}{$\begin{array}{c}\text { Collision strength } \Omega \\
\text { Scattered electron energy }(\mathrm{eV})\end{array}$} \\
\hline & & 10 & 50 & 100 & 200 & 400 & 600 & 800 & 1000 & 1500 & 2000 \\
\hline 15 & 194 & $4.897-05$ & $4.287-05$ & $3.880-05$ & $3.558-05$ & $3.540-05$ & $3.652-05$ & $3.732-05$ & $3.786-05$ & $3.910-05$ & $4.009-05$ \\
\hline 15 & 195 & $1.623-04$ & $1.328-04$ & $1.095-04$ & 8.631-05 & $7.348-05$ & $7.232-05$ & $7.402-05$ & 7.621-05 & $8.062-05$ & $8.317-05$ \\
\hline 15 & 196 & $2.778-04$ & 2.594-04 & $2.500-04$ & $2.540-04$ & $2.832-04$ & $3.111-04$ & $3.356-04$ & $3.549-04$ & $3.851-04$ & $4.002-04$ \\
\hline 15 & 197 & 7.659-05 & 6.941-05 & $6.346-05$ & $5.775-05$ & $5.702-05$ & $6.136-05$ & 6.709-05 & 7.323-05 & $8.755-05$ & 9.969-05 \\
\hline 15 & 198 & $1.204-04$ & 1.154-04 & $1.128-04$ & $1.154-04$ & $1.346-04$ & $1.597-04$ & $1.861-04$ & $2.132-04$ & $2.767-04$ & $3.296-04$ \\
\hline 15 & 199 & $9.315-05$ & $9.952-05$ & $1.093-04$ & $1.312-04$ & $1.733-04$ & 2.093-04 & 2.404-04 & $2.684-04$ & $3.260-04$ & 3.693-04 \\
\hline 15 & 200 & $2.738-04$ & 3.193-04 & $3.786-04$ & 4.948-04 & $6.952-04$ & $8.543-04$ & $9.856-04$ & $1.100-03$ & $1.326-03$ & $1.488-03$ \\
\hline 15 & 201 & $2.523-04$ & $2.679-04$ & $2.886-04$ & 3.329-04 & $4.215-04$ & 5.067-04 & 5.875-04 & 6.659-04 & $8.461-04$ & 9.985-04 \\
\hline 15 & 202 & $9.890-05$ & 9.937-05 & $1.007-04$ & $1.043-04$ & $1.130-04$ & $1.202-04$ & $1.257-04$ & $1.302-04$ & $1.385-04$ & $1.438-04$ \\
\hline 15 & 203 & $2.025-04$ & $2.113-04$ & $2.220-04$ & $2.411-04$ & 2.729-04 & $2.953-04$ & 3.113-04 & $3.237-04$ & $3.459-04$ & 3.597-04 \\
\hline 15 & 204 & $2.434-04$ & $2.426-04$ & $2.446-04$ & $2.531-04$ & 2.759-04 & $2.961-04$ & 3.134-04 & $3.273-04$ & $3.518-04$ & 3.692-04 \\
\hline 15 & 205 & $1.184-04$ & $1.030-04$ & 8.839-05 & $6.918-05$ & $5.074-05$ & $4.264-05$ & 3.883-05 & $3.666-05$ & $3.448-05$ & 3.527-05 \\
\hline 15 & 206 & $5.565-05$ & $5.676-05$ & 5.881-05 & $6.371-05$ & 7.388-05 & $8.260-05$ & $9.021-05$ & $9.658-05$ & $1.073-04$ & $1.139-04$ \\
\hline 15 & 207 & $2.206-04$ & $2.247-04$ & $2.323-04$ & $2.531-04$ & $3.037-04$ & $3.520-04$ & 3.939-04 & $4.293-04$ & 4.951-04 & 5.404-04 \\
\hline 15 & 208 & $7.135-05$ & $6.573-05$ & $6.175-05$ & 5.859-05 & $5.952-05$ & $6.242-05$ & $6.501-05$ & $6.716-05$ & 7.024-05 & 7.114-05 \\
\hline 15 & 209 & $9.214-05$ & 8.644-05 & 8.284-05 & 8.089-05 & $8.474-05$ & 8.975-05 & $9.370-05$ & $9.671-05$ & 9.989-05 & $9.928-05$ \\
\hline 15 & 210 & $2.187-04$ & $1.890-04$ & $1.629-04$ & $1.320-04$ & $1.067-04$ & $9.785-05$ & $9.383-05$ & $9.183-05$ & $9.002-05$ & $8.959-05$ \\
\hline 15 & 211 & $3.120-04$ & $3.073-04$ & $3.068-04$ & $3.114-04$ & $3.297-04$ & $3.461-04$ & $3.558-04$ & $3.623-04$ & $3.730-04$ & $3.780-04$ \\
\hline 15 & 212 & $2.215-04$ & $2.071-04$ & $1.954-04$ & $1.863-04$ & $1.928-04$ & $2.084-04$ & $2.253-04$ & $2.429-04$ & $2.865-04$ & $3.234-04$ \\
\hline 15 & 213 & 8.559-04 & 8.878-04 & $9.312-04$ & $1.028-03$ & $1.223-03$ & $1.404-03$ & $1.567-03$ & $1.720-03$ & $2.066-03$ & $2.355-03$ \\
\hline 15 & 219 & $1.444-05$ & $1.326-05$ & $1.232-05$ & $1.137-05$ & $1.111-05$ & $1.164-05$ & $1.233-05$ & $1.307-05$ & $1.470-05$ & $1.584-05$ \\
\hline 15 & 228 & 4.166-05 & $3.649-05$ & $3.129-05$ & $2.418-05$ & $1.733-05$ & $1.430-05$ & $1.278-05$ & $1.200-05$ & $1.129-05$ & $1.108-05$ \\
\hline 15 & 229 & $2.180-04$ & 2.109-04 & $2.032-04$ & $1.924-04$ & $1.829-04$ & $1.790-04$ & $1.770-04$ & $1.761-04$ & $1.756-04$ & $1.758-04$ \\
\hline 15 & 236 & $1.404-04$ & $1.273-04$ & $1.160-04$ & $1.047-04$ & $1.009-04$ & $1.042-04$ & $1.093-04$ & $1.148-04$ & $1.275-04$ & $1.381-04$ \\
\hline 15 & 237 & $4.900-04$ & 5.037-04 & $5.237-04$ & $5.702-04$ & $6.622-04$ & 7.403-04 & 8.076-04 & 8.668-04 & $9.865-04$ & $1.078-03$ \\
\hline 15 & 243 & $5.880-07$ & $3.823-07$ & $2.417-07$ & $1.214-07$ & $7.302-08$ & $7.722-08$ & $9.228-08$ & $9.147-08$ & $9.505-08$ & $1.833-07$ \\
\hline 15 & 247 & $5.747-05$ & $4.587-05$ & 3.659-05 & $2.724-05$ & $2.177-05$ & $2.102-05$ & $2.150-05$ & $2.222-05$ & $2.370-05$ & $2.457-05$ \\
\hline 15 & 248 & 8.943-05 & 7.961-05 & 7.313-05 & $7.013-05$ & $7.532-05$ & $8.230-05$ & 8.936-05 & $9.525-05$ & $1.045-04$ & $1.091-04$ \\
\hline 15 & 249 & $6.842-06$ & 4.697-06 & $3.118-06$ & $1.636-06$ & 7.698-07 & $5.612-07$ & 4.941-07 & $4.613-07$ & $4.131-07$ & 3.866-07 \\
\hline 15 & 250 & 8.544-06 & 5.891-06 & $3.955-06$ & $2.159-06$ & $1.119-06$ & 8.566-07 & 7.583-07 & 7.133-07 & 6.920-07 & 7.181-07 \\
\hline
\end{tabular}


TABLE IX:

\begin{tabular}{|c|c|c|c|c|c|c|c|c|c|c|c|}
\hline \multirow[t]{3}{*}{$\mathrm{i}$} & \multirow[t]{3}{*}{$\mathrm{j}$} & \multicolumn{10}{|c|}{ Collision strength $\Omega$} \\
\hline & & & & & & ttered elec & n energy ( & & & & \\
\hline & & 10 & 50 & 100 & 200 & 400 & 600 & 800 & 1000 & 1500 & 20 \\
\hline 1 & 2 & $5.681-03$ & $5.114-03$ & $4.499-03$ & $3.539-03$ & $2.325-03$ & $1.634-03$ & $1.208-03$ & $9.291-04$ & $5.441-04$ & 3.55 \\
\hline 1 & 3 & $1.742-02$ & $1.576-02$ & $1.396-02$ & $1.115-02$ & $7.611-03$ & $5.600-03$ & $4.371-03$ & $3.572-03$ & $2.485-03$ & \\
\hline 1 & 4 & $2.818-02$ & $2.537-02$ & $2.233-02$ & $1.756-02$ & $1.154-02$ & 8.104-03 & $5.993-03$ & $4.609-03$ & 2.699-03 & 1.76 \\
\hline 1 & 5 & $1.006+00$ & $1.081+00$ & $1.162+00$ & $1.292+00$ & $1.484+00$ & $1.624+00$ & $1.731+00$ & $1.818+00$ & $1.984+00$ & \\
\hline 1 & 6 & $2.831-04$ & $2.423-04$ & $2.022-04$ & $1.457-04$ & $8.445-05$ & $5.488-05$ & $3.876-05$ & $2.916-05$ & $1.752-05$ & 1.27 \\
\hline 1 & 7 & $8.171-04$ & $6.961-04$ & $5.773-04$ & $4.103-04$ & $2.293-04$ & $1.420-04$ & $9.453-05$ & $6.627-05$ & $3.202-05$ & 1.80 \\
\hline 1 & 8 & $1.362-03$ & $1.168-03$ & $9.784-04$ & 7.116-04 & $4.237-04$ & $2.857-04$ & $2.110-04$ & $1.669-04$ & $1.142-04$ & 9.31 \\
\hline 1 & 9 & $6.221-03$ & $6.642-03$ & 7.076-03 & $7.748-03$ & $8.632-03$ & $9.182-03$ & $9.550-03$ & $9.814-03$ & $1.023-02$ & 1.04 \\
\hline 1 & 10 & $2.189-03$ & $2.164-03$ & $2.137-03$ & $2.092-03$ & $2.031-03$ & $1.990-03$ & $1.964-03$ & $1.947-03$ & $1.922-03$ & \\
\hline 1 & 11 & $5.048-03$ & $4.298-03$ & $3.577-03$ & $2.591-03$ & $1.551-03$ & $1.041-03$ & $7.491-04$ & $5.648-04$ & $3.172-04$ & 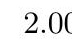 \\
\hline 1 & 12 & $5.047-02$ & 5.234-02 & 5.426-02 & 5.713-02 & $6.058-02$ & $6.257-02$ & $6.383-02$ & $6.468-02$ & $6.591-02$ & \\
\hline 1 & 13 & $1.927-02$ & $2.389-02$ & $2.970-02$ & $4.098-02$ & $6.154-02$ & 7.896-02 & $9.418-02$ & $1.080-01$ & $1.369-01$ & \\
\hline 1 & 14 & $1.100-03$ & $9.537-04$ & 8.077-04 & $6.005-04$ & $3.668-04$ & $2.440-04$ & $1.716-04$ & $1.256-04$ & $6.488-05$ & 3.74 \\
\hline 1 & 15 & $4.206-03$ & $4.073-03$ & $4.010-03$ & 4.113-03 & $4.690-03$ & 5.399-03 & $6.116-03$ & $6.819-03$ & 8.395-03 & 9.67 \\
\hline 1 & 16 & $5.447-03$ & $4.725-03$ & $4.003-03$ & $2.978-03$ & $1.820-03$ & $1.211-03$ & $8.517-04$ & $6.235-04$ & $3.222-04$ & 1.8 \\
\hline 1 & 17 & 6.439-03 & 5.493-03 & $4.568-03$ & $3.290-03$ & $1.930-03$ & $1.262-03$ & $8.858-04$ & $6.534-04$ & $3.531-04$ & 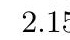 \\
\hline 1 & 18 & $1.073-02$ & $9.174-03$ & $7.628-03$ & 5.494-03 & $3.214-03$ & $2.118-03$ & $1.491-03$ & $1.100-03$ & 5.969-04 & 3.71 \\
\hline 1 & 19 & $1.503-02$ & $1.282-02$ & 1.066-02 & $7.680-03$ & $4.504-03$ & $2.947-03$ & $2.068-03$ & $1.526-03$ & $8.245-04$ & \\
\hline 1 & 20 & $5.673-02$ & 6.346-02 & 7.095-02 & $8.372-02$ & $1.030-01$ & $1.161-01$ & $1.256-01$ & $1.331-01$ & $1.462-01$ & \\
\hline 1 & 21 & 1.704-05 & $1.495-05$ & $1.283-05$ & $9.739-06$ & $6.131-06$ & 4.193-06 & $3.042-06$ & 2.304-06 & $1.308-06$ & \\
\hline 1 & 22 & 6.113-05 & $5.741-05$ & 5.426-05 & 5.134-05 & $5.249-05$ & $5.697-05$ & $6.254-05$ & $6.865-05$ & $8.337-05$ & \\
\hline 1 & 23 & $8.172-05$ & $7.172-05$ & $6.157-05$ & $4.673-05$ & $2.943-05$ & 2.014-05 & $1.462-05$ & $1.108-05$ & $6.297-06$ & \\
\hline 1 & 24 & $6.188-04$ & $7.462-04$ & $9.132-04$ & $1.248-03$ & $1.893-03$ & $2.463-03$ & $2.961-03$ & $3.425-03$ & $4.423-03$ & \\
\hline 1 & 25 & $1.002-04$ & 8.624-05 & 7.247-05 & 5.296-05 & $3.128-05$ & $2.037-05$ & $1.420-05$ & $1.040-05$ & $5.529-06$ & \\
\hline 1 & 26 & $2.096-04$ & $1.836-04$ & $1.574-04$ & $1.193-04$ & $7.482-05$ & $5.114-05$ & $3.706-05$ & $2.805-05$ & $1.597-05$ & \\
\hline 1 & 27 & $3.760-04$ & 3.303-04 & $2.838-04$ & $2.161-04$ & $1.370-04$ & $9.480-05$ & 6.985-05 & 5.377-05 & $3.201-05$ & \\
\hline 1 & 28 & $5.012-04$ & $4.397-04$ & $3.775-04$ & $2.867-04$ & $1.806-04$ & $1.238-04$ & 8.998-05 & $6.823-05$ & $3.897-05$ & 2. \\
\hline 1 & 29 & $9.111-05$ & $8.102-05$ & 7.084-05 & $5.573-05$ & $3.720-05$ & 2.669-05 & 2.014-05 & $1.573-05$ & $9.364-06$ & \\
\hline 1 & 30 & $2.739-05$ & $2.380-05$ & $2.029-05$ & $1.539-05$ & $1.005-05$ & $7.456-06$ & $6.028-06$ & $5.171-06$ & $4.107-06$ & \\
\hline 1 & 31 & $7.300-05$ & $6.230-05$ & 5.186-05 & $3.724-05$ & $2.130-05$ & $1.349-05$ & $9.171-06$ & $6.566-06$ & $3.318-06$ & 1. \\
\hline 1 & 32 & $1.225-04$ & $1.055-04$ & 8.896-05 & $6.602-05$ & $4.154-05$ & $3.004-05$ & $2.385-05$ & $2.025-05$ & $1.616-05$ & \\
\hline 1 & 33 & $2.726-04$ & $2.287-04$ & $1.866-04$ & $1.297-04$ & $7.123-05$ & 4.415-05 & 2.966-05 & $2.110-05$ & $1.063-05$ & \\
\hline 1 & 34 & $4.204-04$ & $3.541-04$ & $2.903-04$ & $2.040-04$ & $1.146-04$ & $7.256-05$ & $5.007-05$ & $3.683-05$ & $2.047-05$ & \\
\hline 1 & 35 & $1.609-04$ & $1.329-04$ & $1.064-04$ & 7.141-05 & $3.693-05$ & $2.179-05$ & $1.406-05$ & $9.699-06$ & 4.689-06 & \\
\hline 1 & 36 & $5.276-04$ & $4.442-04$ & $3.637-04$ & $2.544-04$ & $1.413-04$ & 8.829-05 & $5.970-05$ & $4.266-05$ & $2.162-05$ & \\
\hline 1 & 37 & $3.046-04$ & $3.385-04$ & 3.783-04 & $4.497-04$ & $5.632-04$ & $6.524-04$ & $7.126-04$ & $7.586-04$ & 8.464-04 & \\
\hline 1 & 38 & $1.097-04$ & $9.886-05$ & 8.944-05 & 7.901-05 & $7.390-05$ & $7.601-05$ & 8.054-05 & $8.558-05$ & $9.782-05$ & \\
\hline 1 & 39 & $1.467-04$ & $1.214-04$ & $9.740-05$ & $6.555-05$ & $3.407-05$ & $2.019-05$ & $1.311-05$ & $9.101-06$ & $4.504-06$ & \\
\hline 1 & 40 & $1.667-04$ & $1.365-04$ & $1.083-04$ & $7.154-05$ & $3.648-05$ & $2.174-05$ & $1.463-05$ & $1.084-05$ & $6.805-06$ & \\
\hline 1 & 41 & 3.908-04 & 3.363-04 & $2.822-04$ & $2.058-04$ & $1.219-04$ & 7.995-05 & 5.624-05 & $4.160-05$ & $2.272-05$ & \\
\hline 1 & 42 & $2.450-04$ & $2.125-04$ & $1.804-04$ & $1.352-04$ & 8.649-05 & $6.257-05$ & $4.957-05$ & $4.204-05$ & $3.346-05$ & \\
\hline 1 & 43 & $8.158-05$ & $7.027-05$ & 5.906-05 & $4.315-05$ & $2.563-05$ & $1.685-05$ & $1.187-05$ & 8.793-06 & $4.809-06$ & \\
\hline 1 & 44 & $4.977-04$ & $5.065-04$ & $5.153-04$ & $5.280-04$ & $5.421-04$ & $5.497-04$ & $5.544-04$ & $5.575-04$ & $5.615-04$ & \\
\hline 1 & 45 & $4.379-04$ & 4.974-04 & $5.658-04$ & $6.771-04$ & $8.233-04$ & $9.180-04$ & $9.847-04$ & $1.033-03$ & $1.106-03$ & \\
\hline 1 & 46 & 2.826-03 & 3.151-03 & 3.534-03 & $4.231-03$ & 5.399-03 & $6.331-03$ & $7.140-03$ & 7.835-03 & $9.262-03$ & 1. \\
\hline 1 & 47 & $1.603-03$ & $1.363-03$ & $1.133-03$ & $8.188-04$ & $4.882-04$ & $3.273-04$ & $2.356-04$ & $1.780-04$ & $1.007-04$ & \\
\hline 1 & 48 & $9.359-03$ & $9.757-03$ & $1.018-02$ & $1.082-02$ & $1.163-02$ & $1.212-02$ & $1.244-02$ & $1.267-02$ & $1.300-02$ & 1.31 \\
\hline 1 & 49 & $4.127-04$ & $3.580-04$ & $3.035-04$ & $2.261-04$ & $1.388-04$ & $9.287-05$ & $6.577-05$ & $4.850-05$ & $2.547-05$ & 1.47 \\
\hline 1 & 50 & $1.281-03$ & $1.133-03$ & 9.891-04 & 7.928-04 & 5.939-04 & 5.074-04 & $4.720-04$ & $4.601-04$ & 4.739-04 & \\
\hline 1 & 51 & $2.043-03$ & $1.773-03$ & $1.504-03$ & $1.121-03$ & $6.883-04$ & $4.607-04$ & $3.263-04$ & $2.407-04$ & $1.263-04$ & \\
\hline 1 & 52 & 4.435-03 & 5.443-03 & $6.702-03$ & $9.151-03$ & $1.361-02$ & $1.743-02$ & $2.073-02$ & $2.367-02$ & $3.002-02$ & 3. \\
\hline 1 & 53 & $1.848-03$ & $1.601-03$ & $1.354-03$ & $1.000-03$ & $6.057-04$ & $4.045-04$ & $2.884-04$ & $2.155-04$ & $1.194-04$ & 7. \\
\hline 1 & 54 & $3.079-03$ & $2.668-03$ & $2.258-03$ & $1.669-03$ & $1.012-03$ & $6.742-04$ & $4.824-04$ & $3.627-04$ & $2.032-04$ & 1.2 \\
\hline 1 & 55 & $4.306-03$ & $3.732-03$ & $3.155-03$ & $2.331-03$ & $1.412-03$ & $9.426-04$ & $6.722-04$ & $5.022-04$ & $2.783-04$ & 1.7 \\
\hline 1 & 56 & $1.286-03$ & $1.048-03$ & 8.286-04 & $5.485-04$ & $2.840-04$ & $1.691-04$ & $1.090-04$ & $7.407-05$ & $3.289-05$ & \\
\hline 1 & 57 & $1.790-03$ & $1.453-03$ & $1.150-03$ & $7.722-04$ & $3.999-04$ & $2.382-04$ & $1.548-04$ & $1.063-04$ & $4.881-05$ & 2.6 \\
\hline 1 & 58 & $2.313-03$ & $1.885-03$ & $1.491-03$ & $9.867-04$ & $5.110-04$ & $3.043-04$ & $1.961-04$ & $1.333-04$ & 5.918-05 & 3.01 \\
\hline 1 & 59 & $1.003-02$ & $1.111-02$ & $1.234-02$ & $1.442-02$ & $1.760-02$ & $1.990-02$ & $2.157-02$ & $2.283-02$ & $2.505-02$ & 2. \\
\hline 1 & 60 & 2.613-03 & 3.055-03 & $3.557-03$ & $4.372-03$ & $5.490-03$ & $6.252-03$ & $6.772-03$ & 7.115-03 & $7.548-03$ & 7.71 \\
\hline
\end{tabular}


Table IX-continued ...

\begin{tabular}{|c|c|c|c|c|c|c|c|c|c|c|c|}
\hline \multirow[t]{2}{*}{$\overline{\mathrm{i}}$} & \multirow[t]{2}{*}{$j$} & \multicolumn{10}{|c|}{$\begin{array}{c}\text { Collision strength } \Omega \\
\text { Scattered electron energy }(\mathrm{eV})\end{array}$} \\
\hline & & 10 & 50 & 100 & 200 & 400 & 600 & 800 & 1000 & 1500 & \\
\hline 1 & 61 & $5.912-06$ & $5.247-06$ & $4.553-06$ & $3.513-06$ & $2.258-06$ & $1.563-06$ & $1.142-06$ & $8.694-07$ & $4.964-07$ & \\
\hline 1 & 62 & $1.747-05$ & $1.552-05$ & $1.350-05$ & $1.048-05$ & $6.864-06$ & $4.908-06$ & $3.695-06$ & 2.923-06 & $1.943-06$ & \\
\hline 1 & 63 & $2.860-05$ & 2.539-05 & $2.205-05$ & $1.704-05$ & $1.097-05$ & 7.612-06 & $5.573-06$ & $4.248-06$ & $2.435-06$ & \\
\hline 1 & 64 & $1.263-05$ & $1.114-05$ & 9.669-06 & 7.563-06 & $5.115-06$ & $4.051-06$ & $3.255-06$ & $2.854-06$ & $2.802-06$ & \\
\hline 1 & 65 & $3.974-05$ & $3.444-05$ & 2.919-05 & $2.169-05$ & $1.318-05$ & $8.772-06$ & $6.224-06$ & 4.624-06 & $2.526-06$ & \\
\hline 1 & 66 & $4.085-05$ & $3.546-05$ & $3.012-05$ & $2.247-05$ & $1.375-05$ & $9.211-06$ & $6.572-06$ & $4.903-06$ & $2.698-06$ & \\
\hline 1 & 67 & $7.715-05$ & $6.730-05$ & $5.750-05$ & $4.340-05$ & $2.724-05$ & $1.876-05$ & $1.378-05$ & $1.064-05$ & $6.504-06$ & \\
\hline 1 & 68 & $1.058-04$ & $9.236-05$ & 7.891-05 & 5.949-05 & $3.706-05$ & $2.517-05$ & $1.814-05$ & $1.365-05$ & 7.628-06 & \\
\hline 1 & 69 & $6.203-05$ & $5.445-05$ & $4.686-05$ & $3.578-05$ & $2.279-05$ & $1.580-05$ & $1.161-05$ & 8.876-06 & $5.101-06$ & \\
\hline 1 & 70 & $2.187-05$ & $2.097-05$ & 2.014-05 & $1.906-05$ & $1.805-05$ & $1.768-05$ & $1.755-05$ & $1.752-05$ & $1.755-05$ & \\
\hline 1 & 71 & $9.133-05$ & $7.725-05$ & $6.356-05$ & $4.477-05$ & $2.507-05$ & $1.573-05$ & $1.067-05$ & 7.651-06 & $3.930-06$ & \\
\hline 1 & 72 & $3.893-05$ & $3.372-05$ & $2.857-05$ & $2.122-05$ & $1.289-05$ & $8.605-06$ & $6.129-06$ & $4.571-06$ & $2.514-06$ & \\
\hline 1 & 73 & 4.496-05 & $3.841-05$ & 3.204-05 & $2.315-05$ & $1.355-05$ & 8.934-06 & $6.465-06$ & $5.015-06$ & $3.267-06$ & \\
\hline 1 & 74 & $1.385-04$ & $1.180-04$ & 9.804-05 & $7.070-05$ & $4.215-05$ & $2.863-05$ & $2.131-05$ & $1.699-05$ & $1.177-05$ & \\
\hline 1 & 75 & $7.088-05$ & 5.940-05 & $4.838-05$ & $3.346-05$ & $1.819-05$ & $1.115-05$ & $7.422-06$ & $5.239-06$ & 2.621-06 & \\
\hline 1 & 76 & $1.801-04$ & $1.530-04$ & $1.265-04$ & 8.989-05 & 5.104-05 & $3.237-05$ & $2.214-05$ & $1.598-05$ & $8.299-06$ & \\
\hline 1 & 77 & $1.630-05$ & $1.419-05$ & $1.265-05$ & $1.168-05$ & $1.342-05$ & $1.645-05$ & $1.971-05$ & $2.244-05$ & $2.731-05$ & \\
\hline 1 & 78 & $7.867-05$ & $7.722-05$ & $7.687-05$ & $7.902-05$ & 8.824-05 & $9.849-05$ & $1.085-04$ & $1.178-04$ & $1.382-04$ & \\
\hline 1 & 79 & $2.725-05$ & $2.164-05$ & $1.662-05$ & $1.044-05$ & $4.953-06$ & $2.789-06$ & $1.741-06$ & $1.168-06$ & 5.333-07 & \\
\hline 1 & 80 & $2.068-05$ & $1.641-05$ & $1.254-05$ & 7.777-06 & $3.589-06$ & $1.961-06$ & $1.201-06$ & $7.980-07$ & $3.550-07$ & \\
\hline 1 & 81 & $3.165-05$ & $2.648-05$ & 2.193-05 & $1.683-05$ & $1.350-05$ & $1.272-05$ & $1.257-05$ & $1.265-05$ & $1.321-05$ & \\
\hline 1 & 82 & $8.267-05$ & 6.961-05 & $5.701-05$ & 3.984-05 & $2.206-05$ & $1.375-05$ & $9.265-06$ & $6.609-06$ & $3.368-06$ & \\
\hline 1 & 83 & $1.478-05$ & $1.320-05$ & $1.211-05$ & $1.152-05$ & $1.281-05$ & $1.465-05$ & $1.673-05$ & $1.847-05$ & $2.128-05$ & \\
\hline 1 & 84 & $6.928-05$ & $5.747-05$ & $4.630-05$ & $3.156-05$ & $1.721-05$ & $1.099-05$ & 7.903-06 & $6.212-06$ & 4.401-06 & \\
\hline 1 & 85 & $1.343-04$ & $1.143-04$ & $9.487-05$ & 6.789-05 & $3.912-05$ & $2.514-05$ & $1.737-05$ & $1.264-05$ & $6.653-06$ & \\
\hline 1 & 86 & $9.908-05$ & 8.533-05 & $7.190-05$ & 5.336-05 & $3.382-05$ & $2.468-05$ & $1.976-05$ & $1.695-05$ & $1.399-05$ & \\
\hline 1 & 87 & $2.366-05$ & $1.875-05$ & $1.435-05$ & $8.942-06$ & $4.206-06$ & $2.353-06$ & $1.466-06$ & $9.855-07$ & $4.544-07$ & \\
\hline 1 & 88 & $3.611-05$ & $3.088-05$ & $2.575-05$ & $1.860-05$ & $1.087-05$ & 7.063-06 & $4.922-06$ & $3.605-06$ & 1.916-06 & \\
\hline 1 & 89 & $3.159-05$ & $2.548-05$ & $1.993-05$ & $1.332-05$ & $7.900-06$ & $5.848-06$ & $4.948-06$ & $4.512-06$ & $4.155-06$ & \\
\hline 1 & 90 & 5.797-05 & 4.635-05 & 3.589-05 & $2.291-05$ & $1.116-05$ & $6.368-06$ & $3.987-06$ & $2.663-06$ & $1.185-06$ & \\
\hline 1 & 91 & $3.462-05$ & $3.360-05$ & $3.340-05$ & $3.498-05$ & $4.115-05$ & 4.621-05 & 4.941-05 & $5.180-05$ & 5.661-05 & \\
\hline 1 & 92 & $3.520-05$ & $2.855-05$ & $2.237-05$ & $1.447-05$ & 7.071-06 & 3.939-06 & 2.393-06 & $1.541-06$ & 5.943-07 & \\
\hline 1 & 93 & $\begin{array}{r}2.748-05 \\
\text { available }\end{array}$ & $\begin{array}{l}2.553-05 \\
\text { request }\end{array}$ & $2.428-05$ & 2.398-05 & $2.670-05$ & $3.003-05$ & $3.358-05$ & $3.650-05$ & 4.129-05 & \\
\hline 10 & 296 & $1.686-06$ & $1.202-06$ & $8.202-07$ & $4.171-07$ & $1.461-07$ & $6.578-08$ & $3.299-08$ & $1.752-08$ & $4.327-09$ & \\
\hline 10 & 297 & $4.551-05$ & $3.606-05$ & $2.766-05$ & $1.748-05$ & $8.538-06$ & $4.970-06$ & $3.205-06$ & $2.209-06$ & $1.046-06$ & \\
\hline 10 & 298 & $8.956-06$ & $6.613-06$ & $4.744-06$ & $2.758-06$ & $1.233-06$ & $6.726-07$ & $4.065-07$ & $2.633-07$ & $1.095-07$ & \\
\hline 10 & 299 & $9.659-06$ & 7.588-06 & 5.954-06 & $4.241-06$ & $3.200-06$ & $3.045-06$ & $3.142-06$ & $3.159-06$ & $3.120-06$ & \\
\hline 10 & 300 & $1.532-05$ & $1.474-05$ & $1.483-05$ & $1.610-05$ & $1.919-05$ & $2.147-05$ & $2.289-05$ & $2.388-05$ & $2.517-05$ & \\
\hline 10 & 301 & $1.882-05$ & $1.437-05$ & $1.060-05$ & $6.226-06$ & $2.714-06$ & $1.434-06$ & $8.532-07$ & $5.485-07$ & $2.189-07$ & \\
\hline 10 & 302 & $9.049-05$ & $7.290-05$ & $5.687-05$ & $3.676-05$ & $1.836-05$ & $1.070-05$ & $6.868-06$ & $4.707-06$ & $2.201-06$ & \\
\hline 10 & 303 & 4.906-05 & $4.082-05$ & $3.385-05$ & $2.625-05$ & $2.157-05$ & $2.116-05$ & $2.164-05$ & $2.224-05$ & $2.365-05$ & \\
\hline 10 & 304 & $1.050-04$ & $1.132-04$ & $1.245-04$ & $1.479-04$ & $1.900-04$ & $2.229-04$ & $2.500-04$ & $2.727-04$ & $3.128-04$ & \\
\hline 10 & 305 & $5.762-05$ & $4.962-05$ & $4.176-05$ & $3.063-05$ & $1.819-05$ & $1.191-05$ & 8.351-06 & $6.143-06$ & $3.290-06$ & \\
\hline 10 & 306 & $1.682-04$ & $1.441-04$ & $1.206-04$ & 8.798-05 & $5.230-05$ & $3.447-05$ & $2.431-05$ & $1.797-05$ & $9.650-06$ & \\
\hline 10 & 307 & $1.021-04$ & 8.976-05 & 7.788-05 & $6.171-05$ & 4.511-05 & $3.768-05$ & 3.397-05 & $3.213-05$ & $3.085-05$ & \\
\hline 10 & 308 & $1.112-04$ & $9.756-05$ & $8.388-05$ & $6.388-05$ & $4.038-05$ & $2.782-05$ & $2.033-05$ & $1.548-05$ & $8.818-06$ & \\
\hline 10 & 309 & $1.227-04$ & $1.195-04$ & $1.174-04$ & $1.171-04$ & $1.236-04$ & $1.314-04$ & $1.384-04$ & $1.453-04$ & $1.605-04$ & \\
\hline 10 & 310 & $1.020-03$ & $1.052-03$ & $1.085-03$ & $1.136-03$ & $1.203-03$ & $1.246-03$ & $1.274-03$ & $1.297-03$ & $1.338-03$ & \\
\hline 10 & 311 & $2.017-04$ & $1.691-04$ & $1.381-04$ & $9.642-05$ & $5.369-05$ & $3.382-05$ & $2.309-05$ & $1.669-05$ & 8.767-06 & \\
\hline 10 & 312 & $9.589-05$ & 7.929-05 & $6.368-05$ & $4.310-05$ & $2.274-05$ & $1.368-05$ & 8.977-06 & $6.270-06$ & $3.081-06$ & \\
\hline 10 & 313 & $2.593-08$ & $1.519-08$ & $9.232-09$ & 3.869-09 & $1.028-09$ & $4.231-10$ & $2.072-10$ & $1.150-10$ & $3.730-11$ & \\
\hline 10 & 315 & $1.279-04$ & $1.057-04$ & $8.492-05$ & $5.762-05$ & $3.100-05$ & $1.929-05$ & $1.332-05$ & $9.941-06$ & $6.074-06$ & \\
\hline 10 & 317 & $4.983-08$ & $3.145-08$ & $1.890-08$ & 8.161-09 & 2.827-09 & $1.018-09$ & $3.918-10$ & $1.774-10$ & $5.422-11$ & \\
\hline 10 & 319 & 4.799-08 & $2.751-08$ & $1.817-08$ & 8.597-09 & 2.449-09 & $1.022-09$ & $4.328-10$ & $1.913-10$ & $7.553-11$ & \\
\hline 10 & 320 & $6.457-08$ & $4.130-08$ & $2.532-08$ & $1.145-08$ & $4.261-09$ & $1.533-09$ & 5.391-10 & $2.049-10$ & $6.887-11$ & \\
\hline 10 & 322 & $1.913-04$ & $1.610-04$ & $1.320-04$ & $9.281-05$ & $5.232-05$ & $3.323-05$ & $2.281-05$ & $1.654-05$ & 8.688-06 & \\
\hline 10 & 323 & $1.612-04$ & $1.391-04$ & $1.180-04$ & 8.963-05 & $6.098-05$ & $4.825-05$ & $4.177-05$ & $3.828-05$ & $3.513-05$ & \\
\hline 10 & 324 & 6.383-05 & 5.424-05 & 4.495-05 & $3.220-05$ & 1.871-05 & $1.216-05$ & 8.499-06 & $6.251-06$ & 3.367-06 & \\
\hline
\end{tabular}


Table IX-continued ...

\begin{tabular}{|c|c|c|c|c|c|c|c|c|c|c|c|}
\hline \multirow[t]{2}{*}{$\overline{\mathrm{i}}$} & \multirow[t]{2}{*}{$\bar{j}$} & \multicolumn{10}{|c|}{$\begin{array}{c}\text { Collision strength } \Omega \\
\text { Scattered electron energy }(\mathrm{eV})\end{array}$} \\
\hline & & 10 & 50 & 100 & 200 & 400 & 600 & 800 & 1000 & 1500 & 20 \\
\hline 10 & 325 & $7.484-05$ & $6.608-05$ & $5.964-05$ & $5.481-05$ & $5.803-05$ & $6.500-05$ & $7.160-05$ & $7.719-05$ & $8.797-05$ & 9.60 \\
\hline 10 & 326 & $7.419-07$ & $5.150-07$ & $3.378-07$ & $1.611-07$ & 5.424-08 & $2.467-08$ & $1.325-08$ & 7.877-09 & 2.463-09 & 9 . \\
\hline 10 & 327 & 8.313-07 & $5.825-07$ & $3.882-07$ & $2.079-07$ & $9.187-08$ & $8.816-08$ & $8.961-08$ & $3.965-08$ & $5.175-08$ & \\
\hline 10 & 328 & $1.240-06$ & $9.506-07$ & 7.301-07 & $5.183-07$ & 4.394-07 & $4.076-07$ & 4.397-07 & $4.619-07$ & 4.924-07 & 6.1 \\
\hline 10 & 329 & $1.587-06$ & $1.124-06$ & $7.615-07$ & $3.832-07$ & $1.341-07$ & $6.096-08$ & $3.106-08$ & $1.685-08$ & $4.272-09$ & 1.4 \\
\hline 10 & 330 & $1.086-06$ & 8.136-07 & 5.939-07 & $4.060-07$ & $2.326-07$ & 2.946-07 & 3.394-07 & $1.452-07$ & 2.119-07 & \\
\hline 10 & 331 & $1.485-06$ & $1.091-06$ & $7.524-07$ & $3.888-07$ & $1.309-07$ & $5.177-08$ & $2.496-08$ & $1.445-08$ & $4.776-09$ & \\
\hline 10 & 332 & $1.677-03$ & $1.839-03$ & $2.024-03$ & $2.342-03$ & $2.851-03$ & $3.237-03$ & $3.548-03$ & $3.811-03$ & $4.337-03$ & \\
\hline 10 & 333 & $1.908-06$ & $1.373-06$ & $9.469-07$ & $4.891-07$ & $1.722-07$ & $7.645-08$ & $3.761-08$ & $1.954-08$ & $4.720-09$ & \\
\hline 10 & 334 & $2.379-06$ & $1.848-06$ & $1.447-06$ & $1.054-06$ & 8.886-07 & $8.211-07$ & 8.849-07 & $9.289-07$ & 9.914-07 & 1 . \\
\hline 10 & 335 & $4.279-05$ & $3.332-05$ & $2.502-05$ & $1.512-05$ & $6.820-06$ & $3.742-06$ & $2.330-06$ & $1.585-06$ & $7.662-07$ & \\
\hline 10 & 336 & 5.524-05 & 4.327-05 & $3.278-05$ & $2.029-05$ & $9.988-06$ & $6.212-06$ & $4.580-06$ & $3.746-06$ & $2.859-06$ & \\
\hline 10 & 337 & $8.155-06$ & 5.936-06 & $4.162-06$ & $2.277-06$ & $9.135-07$ & $4.739-07$ & $2.865-07$ & $1.901-07$ & $8.335-08$ & \\
\hline 10 & 338 & $9.501-06$ & 7.074-06 & $5.158-06$ & $3.125-06$ & $1.776-06$ & $1.432-06$ & $1.363-06$ & $1.345-06$ & $1.302-06$ & 1 . \\
\hline 10 & 339 & $1.514-05$ & $1.399-05$ & $1.352-05$ & $1.399-05$ & $1.618-05$ & $1.794-05$ & $1.908-05$ & $1.988-05$ & $2.093-05$ & \\
\hline 10 & 340 & $1.857-05$ & $1.407-05$ & $1.028-05$ & $5.935-06$ & $2.514-06$ & $1.305-06$ & 7.671-07 & 4.893-07 & $1.922-07$ & \\
\hline 10 & 341 & $1.061-04$ & 8.539-05 & $6.656-05$ & $4.295-05$ & $2.137-05$ & $1.242-05$ & $7.942-06$ & $5.426-06$ & $2.510-06$ & 1. \\
\hline 10 & 342 & $9.394-05$ & 7.491-05 & $5.793-05$ & $3.719-05$ & $1.867-05$ & $1.107-05$ & $7.211-06$ & 4.989-06 & $2.351-06$ & 1 . \\
\hline 10 & 343 & $1.073-04$ & $1.040-04$ & $1.032-04$ & $1.075-04$ & $1.234-04$ & $1.390-04$ & $1.528-04$ & $1.649-04$ & $1.873-04$ & \\
\hline 10 & 344 & $1.236-05$ & $1.015-05$ & $8.419-06$ & $6.638-06$ & $5.721-06$ & $5.827-06$ & $6.201-06$ & $6.462-06$ & $6.645-06$ & \\
\hline 10 & 345 & $1.767-05$ & $1.312-05$ & $9.501-06$ & $5.652-06$ & $2.626-06$ & $1.455-06$ & $8.816-07$ & $5.686-07$ & $2.319-07$ & $1.1^{\prime}$ \\
\hline 10 & 346 & $1.908-05$ & $1.468-05$ & $1.091-05$ & $6.481-06$ & $2.862-06$ & $1.512-06$ & 8.916-07 & 5.654-07 & $2.169-07$ & 9 . \\
\hline 10 & 347 & $2.785-05$ & 2.656-05 & 2.636-05 & $2.801-05$ & $3.268-05$ & $3.627-05$ & $3.856-05$ & 4.016-05 & $4.230-05$ & \\
\hline 10 & 348 & $5.421-05$ & $4.826-05$ & $4.381-05$ & $4.047-05$ & $4.241-05$ & $4.680-05$ & $5.057-05$ & $5.351-05$ & $5.876-05$ & 6 . \\
\hline 10 & 349 & $7.880-05$ & $6.411-05$ & $5.055-05$ & $3.326-05$ & $1.703-05$ & $1.006-05$ & 6.494-06 & $4.449-06$ & $2.043-06$ & 1.07 \\
\hline 10 & 350 & $2.400-04$ & 2.663-04 & 2.995-04 & 3.627-04 & 4.699-04 & 5.509-04 & $6.166-04$ & 6.713-04 & 7.693-04 & 8.27 \\
\hline
\end{tabular}


TABLE X:

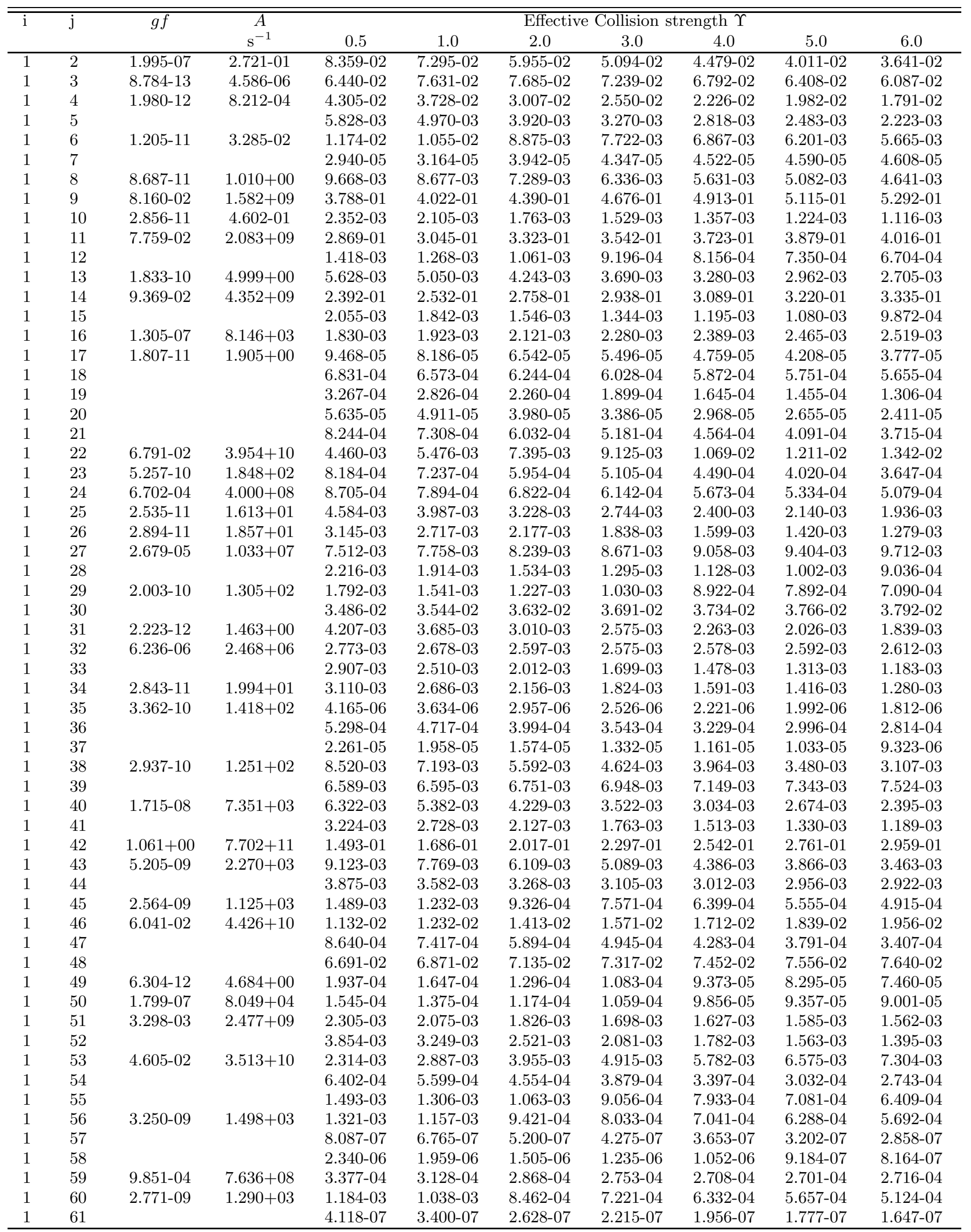


Table X-continued ...

\begin{tabular}{|c|c|c|c|c|c|c|c|c|c|c|}
\hline \multirow[t]{2}{*}{ i } & \multirow[t]{2}{*}{$\bar{j}$} & \multirow[t]{2}{*}{$g f$} & \multirow{2}{*}{$\begin{array}{c}A \\
\mathrm{~s}^{-1}\end{array}$} & \multicolumn{7}{|c|}{ Effective Collision strength $\Upsilon$} \\
\hline & & & & 0.5 & 1.0 & 2.0 & 3.0 & 4.0 & 5.0 & 6.0 \\
\hline 1 & 62 & $1.782-01$ & $1.418+11$ & $6.436-03$ & $8.366-03$ & $1.201-02$ & $1.537-02$ & $1.849-02$ & $2.138-02$ & $2.403-02$ \\
\hline 1 & 63 & $1.067-09$ & $5.101+02$ & $4.254-04$ & $3.722-04$ & $3.018-04$ & $2.582-04$ & $2.227-04$ & $1.911-04$ & $1.644-04$ \\
\hline 1 & 64 & & & $1.183-05$ & $1.233-05$ & $1.315-05$ & $1.379-05$ & $1.434-05$ & $1.480-05$ & $1.518-05$ \\
\hline 1 & 65 & $1.549-09$ & $7.475+02$ & $5.307-04$ & $4.660-04$ & $3.795-04$ & $3.253-04$ & $2.812-04$ & $2.419-04$ & $2.088-04$ \\
\hline 1 & 66 & & & $8.741-05$ & $7.673-05$ & $6.253-05$ & $5.366-05$ & $4.645-05$ & $4.001-05$ & $3.458-05$ \\
\hline 1 & 67 & $7.794-02$ & $6.313+10$ & $2.819-03$ & $3.668-03$ & $5.277-03$ & $6.766-03$ & $8.149-03$ & $9.430-03$ & $1.061-02$ \\
\hline 1 & 68 & $5.959-11$ & $2.899+01$ & $6.977-05$ & $6.135-05$ & $5.012-05$ & $4.310-05$ & $3.737-05$ & $3.226-05$ & $2.794-05$ \\
\hline 1 & 69 & & & $6.618-05$ & 5.799-05 & $4.721-05$ & $4.050-05$ & $3.505-05$ & $3.018-05$ & $2.608-05$ \\
\hline 1 & 70 & $5.209-07$ & $2.557+05$ & $1.967-04$ & $2.107-04$ & $2.342-04$ & $2.536-04$ & $2.706-04$ & 2.854-04 & $2.980-04$ \\
\hline 1 & 71 & $4.959-14$ & $4.056-02$ & 4.804-05 & $4.236-05$ & $3.479-05$ & $3.002-05$ & 2.613-05 & $2.264-05$ & $1.971-05$ \\
\hline 1 & 72 & $2.265-12$ & $1.895+00$ & $3.326-03$ & $2.872-03$ & $2.290-03$ & $1.937-03$ & $1.653-03$ & $1.400-03$ & $1.189-03$ \\
\hline 1 & 73 & $3.107-08$ & $1.561+04$ & $4.083-03$ & $3.526-03$ & 2.813-03 & $2.380-03$ & $2.033-03$ & $1.723-03$ & $1.464-03$ \\
\hline 1 & 74 & & & $2.840-03$ & $2.452-03$ & $1.955-03$ & $1.653-03$ & $1.411-03$ & $1.195-03$ & $1.015-03$ \\
\hline 1 & 75 & & & $6.749-07$ & $5.676-07$ & 4.363-07 & $3.615-07$ & $3.025-07$ & $2.495-07$ & $2.052-07$ \\
\hline 1 & 76 & & & 2.157-06 & $1.815-06$ & $1.398-06$ & $1.160-06$ & $9.722-07$ & 8.034-07 & $6.623-07$ \\
\hline 1 & 77 & $1.647-09$ & $8.338+02$ & $1.113-07$ & $1.085-07$ & $1.050-07$ & $1.033-07$ & $1.019-07$ & $1.006-07$ & 9.951-08 \\
\hline 1 & 78 & & & $4.250-04$ & $3.669-04$ & $2.925-04$ & $2.474-04$ & $2.112-04$ & $1.789-04$ & $1.519-04$ \\
\hline 1 & 79 & $4.361-13$ & 3.697-01 & $1.143-05$ & $9.975-06$ & $8.087-06$ & 6.934-06 & $6.005-06$ & $5.178-06$ & $4.487-06$ \\
\hline 1 & 80 & $1.500-07$ & $7.633+04$ & $1.860-03$ & $1.610-03$ & $1.290-03$ & $1.096-03$ & 9.409-04 & 8.024-04 & $6.867-04$ \\
\hline 1 & 81 & & & $3.470-03$ & 2.995-03 & $2.387-03$ & $2.018-03$ & $1.722-03$ & $1.458-03$ & $1.237-03$ \\
\hline 1 & 82 & & & 4.309-07 & $3.545-07$ & $2.638-07$ & $2.152-07$ & $1.775-07$ & $1.433-07$ & $1.147-07$ \\
\hline 1 & 83 & & & $2.028-03$ & $1.746-03$ & $1.386-03$ & $1.169-03$ & $9.955-04$ & $8.404-04$ & 7.109-04 \\
\hline 1 & 84 & $7.245-07$ & $3.736+05$ & $5.251-04$ & 4.884-04 & $4.465-04$ & $4.259-04$ & 4.105-04 & $3.965-04$ & $3.851-04$ \\
\hline 1 & 85 & $6.828-13$ & 5.875-01 & $2.595-05$ & $2.231-05$ & $1.768-05$ & $1.491-05$ & $1.268-05$ & $1.069-05$ & $9.032-06$ \\
\hline 1 & 86 & $5.088-05$ & $2.631+07$ & $9.502-03$ & $1.054-02$ & $1.218-02$ & $1.347-02$ & $1.458-02$ & $1.556-02$ & $1.638-02$ \\
\hline 1 & 87 & $6.628-14$ & $5.726-02$ & $1.773-04$ & $1.527-04$ & $1.213-04$ & $1.023-04$ & $8.710-05$ & 7.353-05 & $6.220-05$ \\
\hline 1 & 88 & & & $1.042-04$ & $1.063-04$ & $1.096-04$ & $1.121-04$ & $1.141-04$ & $1.158-04$ & $1.172-04$ \\
\hline 1 & 89 & $1.097-04$ & $5.752+07$ & $1.944-02$ & $2.167-02$ & $2.517-02$ & $2.791-02$ & $3.027-02$ & $3.234-02$ & $3.410-02$ \\
\hline 1 & 90 & & & $9.751-04$ & 8.414-04 & $6.703-04$ & $5.666-04$ & $4.833-04$ & 4.091-04 & $3.471-04$ \\
\hline 1 & 91 & & & $1.732-06$ & $1.725-06$ & $1.780-06$ & $1.867-06$ & $1.949-06$ & $2.020-06$ & $2.080-06$ \\
\hline 1 & 92 & $6.364-12$ & $5.635+00$ & $4.065-05$ & 3.504-05 & $2.790-05$ & $2.362-05$ & $2.018-05$ & $1.710-05$ & $1.452-05$ \\
\hline 1 & 93 & $4.486-11$ & $2.396+01$ & $5.774-05$ & 4.901-05 & $3.814-05$ & $3.175-05$ & $2.666-05$ & $2.213-05$ & $1.836-05$ \\
\hline 1 & 94 & & & $3.036-05$ & $2.872-05$ & $2.752-05$ & $2.755-05$ & $2.778-05$ & 2.798-05 & $2.822-05$ \\
\hline 1 & 95 & & & $2.862-05$ & $2.450-05$ & $1.930-05$ & $1.619-05$ & $1.370-05$ & $1.148-05$ & $9.635-06$ \\
\hline 1 & 96 & $6.087-12$ & $3.260+00$ & 3.599-04 & 3.093-04 & $2.449-04$ & $2.062-04$ & $1.752-04$ & $1.475-04$ & $1.245-04$ \\
\hline 1 & 97 & $\begin{array}{l}5.764-13 \\
\text { available }\end{array}$ & $\begin{array}{l}5.165-01 \\
\text { request }\end{array}$ & $1.074-02$ & $1.202-02$ & $1.404-02$ & $1.561-02$ & $1.696-02$ & $1.816-02$ & $1.917-02$ \\
\hline 20 & 454 & & & $9.911-07$ & $1.037-06$ & $1.206-06$ & $1.414-06$ & $1.612-06$ & $1.786-06$ & $1.941-06$ \\
\hline 20 & 455 & $1.219-07$ & $4.969+04$ & $2.635-06$ & $2.271-06$ & $1.804-06$ & $1.524-06$ & $1.299-06$ & $1.098-06$ & $9.291-07$ \\
\hline 20 & 456 & & & 7.287-06 & 6.493-06 & $5.387-06$ & 4.671-06 & $4.081-06$ & $3.555-06$ & $3.112-06$ \\
\hline 20 & 457 & $1.060-14$ & 7.237-03 & $1.054-04$ & $1.066-04$ & $1.088-04$ & $1.108-04$ & $1.126-04$ & $1.140-04$ & $1.152-04$ \\
\hline 20 & 458 & $6.790-13$ & 4.644-01 & 3.931-06 & 3.386-06 & 2.693-06 & 2.284-06 & $1.957-06$ & $1.665-06$ & $1.421-06$ \\
\hline 20 & 459 & & & $1.858-05$ & $1.597-05$ & $1.267-05$ & $1.070-05$ & $9.127-06$ & $7.714-06$ & $6.530-06$ \\
\hline 20 & 460 & & & $2.487-05$ & $2.129-05$ & $1.679-05$ & $1.412-05$ & $1.199-05$ & $1.008-05$ & $8.488-06$ \\
\hline 20 & 461 & & & $2.148-05$ & $2.153-05$ & $2.191-05$ & $2.249-05$ & $2.304-05$ & $2.350-05$ & 2.391-05 \\
\hline 20 & 462 & $2.074-11$ & $8.535+00$ & $1.242-04$ & $1.213-04$ & $1.214-04$ & $1.252-04$ & $1.296-04$ & $1.338-04$ & $1.381-04$ \\
\hline 20 & 463 & & & $1.141-04$ & $9.706-05$ & 7.588-05 & $6.347-05$ & 5.359-05 & $4.476-05$ & 3.739-05 \\
\hline 20 & 464 & $1.697-03$ & $1.164+09$ & $5.745-06$ & $5.040-06$ & $4.098-06$ & $3.509-06$ & $3.031-06$ & $2.604-06$ & $2.245-06$ \\
\hline 20 & 465 & $4.434-10$ & $1.825+02$ & $2.004-05$ & $1.696-05$ & $1.318-05$ & $1.098-05$ & $9.240-06$ & 7.679-06 & $6.378-06$ \\
\hline 20 & 466 & $6.656-07$ & $2.741+05$ & $1.507-05$ & $1.283-05$ & $1.022-05$ & $8.810-06$ & $7.724-06$ & $6.750-06$ & $5.947-06$ \\
\hline 20 & 467 & & & $2.335-05$ & 2.049-05 & $1.668-05$ & $1.431-05$ & $1.238-05$ & $1.065-05$ & $9.205-06$ \\
\hline 20 & 468 & $2.044-12$ & $1.405+00$ & $3.445-04$ & $3.896-04$ & $4.667-04$ & 5.329-04 & 5.931-04 & $6.484-04$ & $6.986-04$ \\
\hline 20 & 469 & $2.045-10$ & $8.431+01$ & $8.462-06$ & $7.425-06$ & 6.093-06 & $5.299-06$ & $4.663-06$ & 4.095-06 & $3.620-06$ \\
\hline 20 & 471 & 8.417-09 & $3.479+03$ & $1.606-04$ & $1.612-04$ & $1.625-04$ & $1.639-04$ & $1.651-04$ & $1.661-04$ & $1.668-04$ \\
\hline 20 & 472 & 5.705-02 & $3.932+10$ & $4.382-04$ & $5.243-04$ & 6.779-04 & $8.158-04$ & 9.434-04 & $1.062-03$ & $1.172-03$ \\
\hline 20 & 473 & & & 6.928-08 & $5.498-08$ & $4.325-08$ & $3.900-08$ & 3.491-08 & 2.925-08 & $2.251-08$ \\
\hline 20 & 474 & & & $3.612-08$ & $2.689-08$ & $1.780-08$ & $1.349-08$ & $1.032-08$ & 7.403-09 & 4.984-09 \\
\hline 20 & 475 & & & 7.667-08 & $5.724-08$ & $3.810-08$ & $2.882-08$ & $2.196-08$ & $1.572-08$ & $1.061-08$ \\
\hline 20 & 476 & & & $4.164-08$ & $3.361-08$ & $2.438-08$ & $1.676-08$ & $1.008-08$ & 4.159-09 & $* * * * * * * *$ \\
\hline 20 & 477 & & & $1.648-05$ & $1.355-05$ & $1.014-05$ & 8.257-06 & 6.791-06 & 5.475-06 & 4.384-06 \\
\hline
\end{tabular}


Table X-continued ...

\begin{tabular}{|c|c|c|c|c|c|c|c|c|c|c|}
\hline \multirow[t]{2}{*}{$\mathrm{i}$} & \multirow[t]{2}{*}{$\mathrm{j}$} & \multirow[t]{2}{*}{$g f$} & \multirow{2}{*}{$\begin{array}{c}A \\
\mathrm{~s}^{-1}\end{array}$} & \multicolumn{7}{|c|}{ Effective Collision strength $\Upsilon$} \\
\hline & & & & 0.5 & 1.0 & 2.0 & 3.0 & 4.0 & 5.0 & 6.0 \\
\hline 20 & 478 & & & $2.898-05$ & $3.102-05$ & $3.464-05$ & $3.770-05$ & $4.038-05$ & $4.272-05$ & $4.471-05$ \\
\hline 20 & 479 & & & $6.452-06$ & $5.200-06$ & $3.792-06$ & $3.044-06$ & $2.468-06$ & $1.948-06$ & $1.517-06$ \\
\hline 20 & 480 & & & 7.294-06 & $6.168-06$ & $4.992-06$ & $4.437-06$ & $4.029-06$ & $3.656-06$ & $3.350-06$ \\
\hline 20 & 481 & $2.027-08$ & $8.397+03$ & $1.955-06$ & $1.678-06$ & $1.323-06$ & $1.111-06$ & $9.421-07$ & $7.907-07$ & $6.641-07$ \\
\hline 20 & 482 & & & $4.634-06$ & $4.649-06$ & $4.774-06$ & $4.917-06$ & $5.044-06$ & $5.147-06$ & $5.226-06$ \\
\hline 20 & 483 & & & $3.986-06$ & $3.190-06$ & $2.306-06$ & $1.839-06$ & $1.481-06$ & $1.158-06$ & 8.911-07 \\
\hline 20 & 484 & & & $6.887-07$ & $5.297-07$ & $3.653-07$ & $2.831-07$ & $2.213-07$ & $1.654-07$ & 1.193-07 \\
\hline 20 & 485 & & & $8.535-07$ & $6.733-07$ & $4.879-07$ & $3.963-07$ & $3.277-07$ & $2.653-07$ & $2.138-07$ \\
\hline 20 & 486 & & & $2.494-05$ & $2.099-05$ & $1.620-05$ & $1.343-05$ & $1.124-05$ & $9.282-06$ & 7.655-06 \\
\hline 20 & 487 & $6.702-12$ & $2.781+00$ & $1.270-05$ & $1.060-05$ & 8.089-06 & $6.654-06$ & $5.525-06$ & $4.515-06$ & $3.677-06$ \\
\hline 20 & 488 & & & $1.655-05$ & $1.377-05$ & $1.046-05$ & 8.601-06 & 7.143-06 & $5.838-06$ & 4.757-06 \\
\hline 20 & 489 & $2.323-14$ & $1.608-02$ & $2.438-05$ & $2.072-05$ & $1.622-05$ & $1.355-05$ & $1.143-05$ & $9.541-06$ & 7.968-06 \\
\hline 20 & 490 & $1.240-11$ & $5.153+00$ & $2.219-05$ & $1.978-05$ & $1.692-05$ & $1.534-05$ & $1.412-05$ & $1.304-05$ & $1.216-05$ \\
\hline 20 & 491 & $6.203-05$ & $4.296+07$ & 7.807-06 & 6.693-06 & $5.297-06$ & $4.458-06$ & $3.786-06$ & $3.187-06$ & 2.689-06 \\
\hline 20 & 492 & & & $1.066-05$ & $9.797-06$ & $9.192-06$ & $9.220-06$ & $9.349-06$ & $9.453-06$ & $9.568-06$ \\
\hline 20 & 493 & & & $3.793-04$ & $4.108-04$ & $4.623-04$ & $5.043-04$ & $5.417-04$ & $5.754-04$ & $6.052-04$ \\
\hline 20 & 494 & $9.102-04$ & $6.318+08$ & $3.514-08$ & $2.590-08$ & $1.705-08$ & $1.278-08$ & $9.631-09$ & $6.780-09$ & 4.454-09 \\
\hline 20 & 495 & & & $4.132-08$ & $3.147-08$ & $2.156-08$ & $1.558-08$ & $1.078-08$ & 6.479-09 & 2.873-09 \\
\hline 20 & 496 & & & $5.887-08$ & $4.586-08$ & $3.471-08$ & $3.033-08$ & $2.647-08$ & $2.167-08$ & $1.633-08$ \\
\hline 20 & 497 & $1.067-14$ & $4.451-03$ & $6.713-08$ & $4.988-08$ & $3.304-08$ & $2.489-08$ & $1.886-08$ & $1.340-08$ & 8.945-09 \\
\hline 20 & 498 & & & 7.161-06 & $5.751-06$ & $4.173-06$ & $3.339-06$ & $2.698-06$ & $2.119-06$ & $1.640-06$ \\
\hline 20 & 499 & & & $4.811-08$ & $4.024-08$ & $3.032-08$ & $2.059-08$ & $1.171-08$ & 3.845-09 & $* * * * * * * *$ \\
\hline 20 & 500 & & & 5.669-08 & $4.249-08$ & $2.825-08$ & $2.155-08$ & $1.660-08$ & $1.203-08$ & 8.220-09 \\
\hline 20 & 501 & & & $1.103-07$ & $8.458-08$ & $5.812-08$ & $4.495-08$ & $3.508-08$ & $2.613-08$ & $1.875-08$ \\
\hline 20 & 502 & & & $1.220-07$ & $9.455-08$ & 6.954-08 & $5.881-08$ & $4.945-08$ & $3.841-08$ & 2.654-08 \\
\hline 20 & 503 & & & $7.388-06$ & $5.958-06$ & $4.403-06$ & $3.612-06$ & $3.013-06$ & $2.470-06$ & $2.022-06$ \\
\hline 20 & 504 & & & $1.747-05$ & $1.437-05$ & $1.076-05$ & 8.763-06 & $7.211-06$ & $5.817-06$ & 4.660-06 \\
\hline 20 & 505 & & & $2.013-05$ & $1.904-05$ & $1.820-05$ & $1.809-05$ & $1.811-05$ & $1.811-05$ & $1.811-05$ \\
\hline 20 & 506 & & & $1.426-05$ & $1.183-05$ & 8.956-06 & $7.343-06$ & $6.079-06$ & $4.946-06$ & 4.006-06 \\
\hline 20 & 507 & & & $1.129-05$ & $9.135-06$ & $6.702-06$ & $5.400-06$ & $4.396-06$ & $3.490-06$ & $2.738-06$ \\
\hline 20 & 508 & & & $5.670-05$ & $6.220-05$ & 7.124-05 & 7.854-05 & $8.486-05$ & $9.039-05$ & $9.507-05$ \\
\hline 20 & 509 & & & $8.252-06$ & $7.383-06$ & $6.587-06$ & $6.313-06$ & $6.143-06$ & $5.979-06$ & $5.848-06$ \\
\hline 20 & 510 & & & $3.773-06$ & $3.679-06$ & $3.658-06$ & $3.704-06$ & $3.752-06$ & $3.787-06$ & 3.813-06 \\
\hline 20 & 511 & & & $3.208-06$ & $2.543-06$ & $1.815-06$ & $1.436-06$ & $1.147-06$ & 8.859-07 & $6.702-07$ \\
\hline 20 & 512 & 4.516-09 & $1.886+03$ & $3.656-06$ & 2.917-06 & 2.099-06 & $1.669-06$ & $1.340-06$ & $1.043-06$ & 7.969-07 \\
\hline 20 & 513 & $2.703-14$ & $1.881-02$ & $7.917-06$ & $7.852-06$ & $7.947-06$ & $8.120-06$ & $8.280-06$ & $8.404-06$ & $8.500-06$ \\
\hline 20 & 514 & & & 7.434-07 & $5.688-07$ & 3.894-07 & $3.001-07$ & $2.332-07$ & $1.726-07$ & $1.228-07$ \\
\hline 20 & 515 & & & $9.109-07$ & $7.072-07$ & $5.001-07$ & $3.990-07$ & $3.238-07$ & $2.554-07$ & 1.991-07 \\
\hline 20 & 516 & $3.592-08$ & $1.501+04$ & $1.048-06$ & $8.515-07$ & $6.512-07$ & $5.565-07$ & $4.865-07$ & $4.219-07$ & $3.680-07$ \\
\hline 20 & 517 & & & $1.220-06$ & $9.471-07$ & $6.616-07$ & $5.169-07$ & $4.078-07$ & $3.090-07$ & $2.276-07$ \\
\hline 20 & 518 & & & $4.335-07$ & $3.749-07$ & $2.997-07$ & $2.542-07$ & $2.176-07$ & $1.849-07$ & $1.575-07$ \\
\hline 20 & 519 & & & $9.013-06$ & $1.068-05$ & $1.370-05$ & $1.644-05$ & $1.897-05$ & 2.134-05 & $2.352-05$ \\
\hline 20 & 520 & $1.053-03$ & $7.458+08$ & $1.678-06$ & $1.423-06$ & $1.108-06$ & $9.250-07$ & 7.795-07 & $6.492-07$ & 5.405-07 \\
\hline 20 & 521 & 9.034-12 & $3.844+00$ & $9.499-05$ & $8.197-05$ & $6.528-05$ & $5.522-05$ & $4.713-05$ & 3.991-05 & 3.387-05 \\
\hline 20 & 522 & $2.878-14$ & 2.044-02 & $1.630-04$ & $1.410-04$ & $1.128-04$ & $9.575-05$ & $8.207-05$ & $6.987-05$ & $5.967-05$ \\
\hline 20 & 523 & $5.270-09$ & $2.246+03$ & $2.325-04$ & $2.008-04$ & $1.600-04$ & $1.354-04$ & $1.157-04$ & $9.800-05$ & 8.323-05 \\
\hline 20 & 524 & & & $6.052-07$ & $5.035-07$ & 3.854-07 & $3.215-07$ & $2.720-07$ & $2.277-07$ & $1.910-07$ \\
\hline 20 & 525 & & & $5.566-07$ & $4.885-07$ & $3.977-07$ & $3.406-07$ & $2.942-07$ & $2.528-07$ & $2.181-07$ \\
\hline 20 & 526 & & & $3.951-06$ & $4.213-06$ & $4.769-06$ & $5.342-06$ & $5.895-06$ & $6.420-06$ & $6.918-06$ \\
\hline 20 & 527 & $2.227-05$ & $1.588+07$ & $3.431-07$ & $2.976-07$ & $2.393-07$ & $2.033-07$ & $1.742-07$ & $1.484-07$ & $1.269-07$ \\
\hline 20 & 528 & $5.271-11$ & $2.260+01$ & $1.012-03$ & $1.110-03$ & $1.264-03$ & $1.386-03$ & $1.491-03$ & $1.583-03$ & $1.662-03$ \\
\hline 20 & 529 & $4.784-06$ & $2.054+06$ & $1.458-07$ & $1.378-07$ & $1.281-07$ & $1.222-07$ & $1.176-07$ & $1.134-07$ & $1.099-07$ \\
\hline 20 & 530 & 7.924-05 & $5.687+07$ & $6.882-07$ & $5.783-07$ & $4.448-07$ & $3.684-07$ & $3.080-07$ & $2.540-07$ & $2.090-07$ \\
\hline 20 & 531 & $8.235-11$ & $3.554+01$ & $8.616-05$ & $7.070-05$ & $5.264-05$ & $4.276-05$ & $3.507-05$ & $2.815-05$ & $2.240-05$ \\
\hline 20 & 532 & $1.812-12$ & $7.860-01$ & $1.204-04$ & 9.904-05 & $7.406-05$ & $6.023-05$ & $4.946-05$ & 3.979-05 & 3.179-05 \\
\hline 20 & 533 & & & $1.557-04$ & $1.278-04$ & $9.518-05$ & $7.733-05$ & $6.344-05$ & 5.093-05 & $4.052-05$ \\
\hline 20 & 534 & & & $3.745-04$ & $4.213-04$ & $4.863-04$ & $5.313-04$ & $5.681-04$ & 5.995-04 & $6.249-04$ \\
\hline 20 & 535 & & & $8.902-06$ & 8.394-06 & $7.813-06$ & 7.514-06 & $7.290-06$ & $7.089-06$ & 6.926-06 \\
\hline 20 & 536 & $1.257-02$ & $9.140+09$ & $2.588-05$ & $2.828-05$ & $3.208-05$ & $3.508-05$ & $3.768-05$ & 3.996-05 & 4.191-05 \\
\hline 20 & 537 & $1.082-07$ & $4.757+04$ & $1.616-06$ & $1.377-06$ & $1.078-06$ & $9.026-07$ & 7.625-07 & $6.374-07$ & 5.329-07 \\
\hline
\end{tabular}


Table X-continued ...

\begin{tabular}{|c|c|c|c|c|c|c|c|c|c|c|}
\hline \multirow[t]{2}{*}{$\overline{\mathrm{i}}$} & \multirow[t]{2}{*}{$\overline{\mathrm{j}}$} & \multirow[t]{2}{*}{$\overline{g f}$} & \multirow{2}{*}{$\begin{array}{c}A \\
\mathrm{~s}^{-1}\end{array}$} & \multicolumn{7}{|c|}{ Effective Collision strength $\Upsilon$} \\
\hline & & & & 0.5 & 1.0 & 2.0 & 3.0 & 4.0 & 5.0 & 6.0 \\
\hline 20 & 539 & & & $7.781-08$ & $6.343-08$ & $4.876-08$ & $4.199-08$ & $3.709-08$ & $3.261-08$ & $2.895-08$ \\
\hline 20 & 541 & $3.242-08$ & $1.434+04$ & $3.112-06$ & $2.736-06$ & $2.237-06$ & $1.923-06$ & $1.666-06$ & $1.438-06$ & $1.246-06$ \\
\hline 20 & 542 & & & $2.228-06$ & $1.997-06$ & $1.676-06$ & $1.463-06$ & $1.287-06$ & $1.131-06$ & $9.982-07$ \\
\hline 20 & 543 & & & $7.264-07$ & $6.559-07$ & $5.602-07$ & $4.986-07$ & $4.483-07$ & $4.040-07$ & $3.670-07$ \\
\hline 20 & 546 & & & $8.678-07$ & $7.471-07$ & 5.944-07 & $5.028-07$ & $4.294-07$ & $3.637-07$ & $3.087-07$ \\
\hline 20 & 547 & & & $1.444-06$ & $1.191-06$ & $8.922-07$ & $7.276-07$ & 5.991-07 & $4.835-07$ & $3.874-07$ \\
\hline 20 & 548 & $9.335-11$ & $4.187+01$ & $4.052-06$ & $4.304-06$ & $4.691-06$ & $4.984-06$ & $5.230-06$ & $5.437-06$ & 5.603-06 \\
\hline 20 & 549 & & & $3.868-06$ & $4.046-06$ & $4.335-06$ & $4.566-06$ & $4.760-06$ & $4.922-06$ & $5.050-06$ \\
\hline 20 & 550 & & & $2.367-06$ & $1.935-06$ & $1.435-06$ & $1.163-06$ & $9.511-07$ & $7.607-07$ & $6.023-07$ \\
\hline 20 & 551 & & & $3.604-07$ & $4.372-07$ & $5.724-07$ & $6.870-07$ & $7.890-07$ & $8.801-07$ & $9.600-07$ \\
\hline 20 & 556 & & & $4.874-07$ & $4.084-07$ & $3.123-07$ & $2.576-07$ & $2.145-07$ & $1.759-07$ & $1.436-07$ \\
\hline 20 & 557 & 7.979-12 & $3.609+00$ & $1.091-06$ & $1.148-06$ & $1.233-06$ & $1.298-06$ & $1.351-06$ & $1.396-06$ & $1.432-06$ \\
\hline 20 & 558 & & & $1.466-07$ & $1.173-07$ & $8.471-08$ & $6.748-08$ & $5.427-08$ & $4.236-08$ & $3.252-08$ \\
\hline 20 & 559 & & & $2.722-07$ & $2.285-07$ & $1.816-07$ & $1.587-07$ & $1.418-07$ & $1.264-07$ & $1.138-07$ \\
\hline
\end{tabular}


TABLE XI:

\begin{tabular}{|c|c|c|c|c|c|c|c|c|c|c|}
\hline \multirow[t]{2}{*}{$\bar{i}$} & \multirow[t]{2}{*}{$\mathrm{j}$} & \multirow{2}{*}{$\overline{\overline{g f f}}$} & \multirow{2}{*}{$\begin{array}{c}A \\
\mathrm{~s}^{-1}\end{array}$} & \multicolumn{7}{|c|}{$\overline{\text { Effective Collision strength } \Upsilon}$} \\
\hline & & & & 0.5 & 1.0 & 2.0 & 3.0 & 4.0 & 5.0 & 6.0 \\
\hline 1 & 2 & $3.683-07$ & $2.871+00$ & $1.899-01$ & $1.966-01$ & $1.862-01$ & $1.717-01$ & $1.591-01$ & $1.486-01$ & $1.400-01$ \\
\hline 1 & 3 & $3.261-05$ & $2.691+05$ & $1.523-02$ & $1.389-02$ & $1.192-02$ & $1.052-02$ & $9.472-03$ & $8.649-03$ & $7.982-03$ \\
\hline 1 & 4 & $1.404-06$ & $5.983+03$ & $2.130-02$ & $1.936-02$ & $1.648-02$ & $1.445-02$ & $1.292-02$ & $1.172-02$ & $1.074-02$ \\
\hline 1 & 5 & $2.090-11$ & $6.194-02$ & $1.277-02$ & $1.160-02$ & $9.875-03$ & $8.655-03$ & $7.737-03$ & $7.015-03$ & $6.430-03$ \\
\hline 1 & 6 & $2.173-10$ & $2.061+00$ & $2.356-02$ & $2.140-02$ & $1.824-02$ & $1.600-02$ & $1.431-02$ & $1.299-02$ & 1.191-02 \\
\hline 1 & 7 & $1.421-01$ & $2.022+09$ & $6.756-01$ & 7.173-01 & 7.821-01 & $8.325-01$ & $8.741-01$ & 9.097-01 & 9.409-01 \\
\hline 1 & 8 & $1.126-01$ & $5.238+09$ & $3.874-01$ & $4.101-01$ & $4.459-01$ & $4.743-01$ & $4.980-01$ & 5.184-01 & 5.364-01 \\
\hline 1 & 9 & $1.900-01$ & $1.007+10$ & 5.924-01 & $6.271-01$ & $6.822-01$ & $7.261-01$ & $7.628-01$ & 7.944-01 & $8.224-01$ \\
\hline 1 & 10 & $1.103-01$ & $2.981+09$ & $3.437-01$ & $3.632-01$ & $3.943-01$ & $4.192-01$ & $4.400-01$ & $4.580-01$ & 4.739-01 \\
\hline 1 & 11 & $6.767-11$ & $6.494+00$ & $9.835-04$ & $8.565-04$ & 6.903-04 & 5.836-04 & $5.080-04$ & $4.511-04$ & $4.065-04$ \\
\hline 1 & 12 & $7.770-11$ & $8.860+03$ & $3.932-03$ & $4.216-03$ & $4.850-03$ & $5.283-03$ & 5.544-03 & $5.706-03$ & $5.811-03$ \\
\hline 1 & 13 & $9.541-08$ & $3.586+03$ & $3.137-03$ & $3.211-03$ & $3.466-03$ & $3.644-03$ & $3.744-03$ & $3.797-03$ & $3.826-03$ \\
\hline 1 & 14 & & & $2.886-03$ & 2.839-03 & $2.771-03$ & $2.724-03$ & 2.689-03 & $2.662-03$ & $2.640-03$ \\
\hline 1 & 15 & $1.092-10$ & $6.431+03$ & $1.894-03$ & $1.934-03$ & $2.029-03$ & $2.119-03$ & $2.186-03$ & $2.235-03$ & $2.270-03$ \\
\hline 1 & 16 & $5.113-02$ & $5.616+10$ & $5.364-03$ & 5.699-03 & $6.485-03$ & $7.295-03$ & $8.082-03$ & $8.833-03$ & $9.547-03$ \\
\hline 1 & 17 & $1.320-11$ & $1.585+01$ & $4.599-02$ & $4.590-02$ & $4.576-02$ & $4.569-02$ & $4.565-02$ & $4.562-02$ & $4.560-02$ \\
\hline 1 & 18 & $1.998-10$ & $2.358+07$ & $2.110-02$ & $1.993-02$ & $1.852-02$ & $1.773-02$ & $1.726-02$ & $1.696-02$ & $1.677-02$ \\
\hline 1 & 19 & $1.246+00$ & $8.101+11$ & $1.872-01$ & $2.043-01$ & $2.350-01$ & $2.618-01$ & $2.857-01$ & $3.073-01$ & $3.270-01$ \\
\hline 1 & 20 & $2.224-08$ & $9.649+03$ & $3.363-02$ & $3.017-02$ & $2.593-02$ & $2.340-02$ & $2.172-02$ & $2.052-02$ & $1.963-02$ \\
\hline 1 & 21 & $3.551-11$ & $4.677+01$ & $3.057-03$ & $2.687-03$ & $2.200-03$ & $1.887-03$ & $1.664-03$ & $1.495-03$ & $1.362-03$ \\
\hline 1 & 22 & $5.257-11$ & $4.802+03$ & $3.861-03$ & $3.383-03$ & $2.755-03$ & $2.351-03$ & $2.063-03$ & $1.845-03$ & $1.674-03$ \\
\hline 1 & 23 & $3.057-11$ & $1.351+01$ & $6.840-05$ & 5.987-05 & $4.868-05$ & $4.146-05$ & $3.631-05$ & $3.242-05$ & $2.935-05$ \\
\hline 1 & 24 & $3.146-12$ & $4.326+00$ & $1.080-01$ & $1.110-01$ & $1.151-01$ & $1.180-01$ & $1.201-01$ & $1.218-01$ & $1.232-01$ \\
\hline 1 & 25 & $2.212-11$ & $3.171+05$ & $6.155-04$ & 5.455-04 & $4.563-04$ & $4.011-04$ & 3.634-04 & $3.359-04$ & $3.149-04$ \\
\hline 1 & 26 & 7.384-02 & $1.046+11$ & $5.071-03$ & $5.758-03$ & $7.105-03$ & $8.362-03$ & $9.525-03$ & $1.060-02$ & $1.161-02$ \\
\hline 1 & 27 & $3.442-02$ & $2.442+10$ & $4.317-03$ & $4.402-03$ & $4.707-03$ & $5.073-03$ & $5.454-03$ & $5.831-03$ & $6.198-03$ \\
\hline 1 & 28 & $1.200-01$ & $1.706+11$ & $5.481-03$ & $6.677-03$ & $8.902-03$ & $1.091-02$ & $1.273-02$ & $1.439-02$ & $1.593-02$ \\
\hline 1 & 29 & 7.729-02 & $5.500+10$ & $4.295-03$ & 4.994-03 & $6.335-03$ & $7.570-03$ & $8.705-03$ & $9.753-03$ & $1.073-02$ \\
\hline 1 & 30 & $9.246-09$ & $4.388+03$ & $2.659-03$ & $2.359-03$ & $1.951-03$ & $1.679-03$ & $1.480-03$ & $1.328-03$ & $1.207-03$ \\
\hline 1 & 31 & & & 8.124-06 & 6.905-06 & $5.401-06$ & $4.480-06$ & $3.847-06$ & $3.380-06$ & $3.020-06$ \\
\hline 1 & 32 & 2.124-03 & $1.533+09$ & $1.517-03$ & $1.380-03$ & $1.204-03$ & $1.096-03$ & $1.023-03$ & $9.729-04$ & $9.364-04$ \\
\hline 1 & 33 & $3.626-04$ & $5.303+08$ & $1.291-03$ & $1.153-03$ & $9.652-04$ & $8.410-04$ & $7.515-04$ & $6.834-04$ & $6.297-04$ \\
\hline 1 & 34 & 3.908-04 & $2.863+08$ & $1.417-03$ & $1.266-03$ & $1.062-03$ & $9.264-04$ & $8.289-04$ & $7.549-04$ & $6.965-04$ \\
\hline 1 & 35 & $3.767-09$ & $1.843+03$ & $1.009-03$ & 8.975-04 & $7.444-04$ & $6.415-04$ & $5.665-04$ & $5.087-04$ & $4.625-04$ \\
\hline 1 & 36 & $4.688-01$ & $3.467+11$ & $1.838-02$ & 2.309-02 & $3.186-02$ & 3.977-02 & 4.696-02 & 5.355-02 & 5.965-02 \\
\hline 1 & 37 & $8.158-10$ & $4.040+02$ & $2.691-04$ & 2.426-04 & $2.067-04$ & $1.828-04$ & $1.655-04$ & $1.523-04$ & $1.418-04$ \\
\hline 1 & 38 & $6.598-12$ & $4.163+05$ & $7.248-03$ & 6.365-03 & $5.207-03$ & $4.459-03$ & 3.927-03 & $3.525-03$ & $3.209-03$ \\
\hline 1 & 39 & $1.523-07$ & $7.683+04$ & $8.597-03$ & $7.532-03$ & $6.130-03$ & $5.225-03$ & $4.580-03$ & $4.092-03$ & $3.707-03$ \\
\hline 1 & 40 & & & $6.913-03$ & $6.052-03$ & $4.920-03$ & $4.188-03$ & $3.667-03$ & $3.272-03$ & 2.961-03 \\
\hline 1 & 41 & $8.900-02$ & $1.352+11$ & $3.132-03$ & $3.973-03$ & $5.553-03$ & $6.988-03$ & $8.299-03$ & $9.504-03$ & $1.062-02$ \\
\hline 1 & 42 & & & $4.838-06$ & $4.124-06$ & $3.247-06$ & $2.709-06$ & $2.338-06$ & 2.064-06 & $1.851-06$ \\
\hline 1 & 43 & $2.642-12$ & $4.066+00$ & $2.248-03$ & $1.964-03$ & $1.593-03$ & $1.355-03$ & $1.185-03$ & $1.057-03$ & $9.566-04$ \\
\hline 1 & 44 & $2.840-14$ & $3.857+05$ & $4.777-03$ & 4.197-03 & $3.441-03$ & 2.956-03 & 2.613-03 & $2.355-03$ & $2.153-03$ \\
\hline 1 & 45 & $4.298-06$ & $2.207+06$ & $8.114-03$ & $7.242-03$ & $6.120-03$ & $5.412-03$ & $4.916-03$ & $4.547-03$ & $4.260-03$ \\
\hline 1 & 46 & & & $2.781-03$ & $2.427-03$ & $1.966-03$ & $1.669-03$ & $1.459-03$ & $1.300-03$ & $1.175-03$ \\
\hline 1 & 47 & $2.206-12$ & $9.177+07$ & $1.952-02$ & $2.145-02$ & $2.451-02$ & $2.688-02$ & $2.879-02$ & $3.039-02$ & $3.175-02$ \\
\hline 1 & 48 & 6.989-05 & $3.603+07$ & $1.309-02$ & $1.398-02$ & $1.546-02$ & $1.665-02$ & $1.762-02$ & $1.844-02$ & $1.915-02$ \\
\hline 1 & 49 & $1.505-05$ & $7.787+06$ & $4.167-03$ & $4.246-03$ & $4.424-03$ & $4.592-03$ & $4.744-03$ & $4.879-03$ & $5.000-03$ \\
\hline 1 & 50 & $1.196-12$ & $1.009+06$ & $9.575-04$ & 8.975-04 & $8.260-04$ & $7.853-04$ & 7.596-04 & $7.422-04$ & $7.299-04$ \\
\hline 1 & 51 & $9.514-14$ & $1.479-01$ & $1.176-04$ & $1.058-04$ & $9.043-05$ & $8.064-05$ & $7.372-05$ & $6.852-05$ & $6.445-05$ \\
\hline 1 & 52 & $4.243-12$ & $6.616+00$ & $2.104-02$ & $2.166-02$ & $2.255-02$ & $2.317-02$ & $2.365-02$ & $2.403-02$ & $2.434-02$ \\
\hline 1 & 53 & $4.523-11$ & $3.094+06$ & $2.115-03$ & $1.978-03$ & $1.813-03$ & $1.719-03$ & $1.659-03$ & $1.618-03$ & $1.589-03$ \\
\hline 1 & 54 & $3.010-04$ & $1.606+08$ & $4.611-02$ & 5.095-02 & $5.860-02$ & $6.451-02$ & $6.928-02$ & 7.327-02 & 7.667-02 \\
\hline 1 & 55 & & & $9.240-04$ & 8.079-04 & $6.562-04$ & $5.585-04$ & $4.891-04$ & $4.367-04$ & $3.954-04$ \\
\hline 1 & 56 & 6.269-12 & $8.704+07$ & $1.668-02$ & $1.836-02$ & $2.102-02$ & $2.308-02$ & $2.475-02$ & $2.615-02$ & $2.734-02$ \\
\hline 1 & 57 & $1.927-14$ & $3.115-02$ & $5.614-04$ & $5.338-04$ & $5.002-04$ & 4.804-04 & $4.673-04$ & $4.580-04$ & $4.511-04$ \\
\hline 1 & 58 & $4.519-02$ & $7.572+10$ & $1.537-03$ & $1.880-03$ & $2.549-03$ & $3.173-03$ & $3.752-03$ & $4.290-03$ & $4.792-03$ \\
\hline 1 & 59 & $5.940-04$ & $4.981+08$ & $1.130-03$ & $1.042-03$ & $9.305-04$ & 8.634-04 & $8.201-04$ & $7.910-04$ & 7.713-04 \\
\hline 1 & 60 & $5.742-09$ & $3.214+03$ & $1.813-03$ & $1.633-03$ & $1.377-03$ & $1.201-03$ & $1.070-03$ & $9.687-04$ & $8.871-04$ \\
\hline 1 & 61 & 5.679-02 & $4.768+10$ & $1.673-03$ & $2.062-03$ & $2.838-03$ & $3.571-03$ & $4.256-03$ & 4.895-03 & 5.493-03 \\
\hline
\end{tabular}


Table XI-continued ...

\begin{tabular}{|c|c|c|c|c|c|c|c|c|c|c|}
\hline \multirow[t]{2}{*}{ i } & \multirow[t]{2}{*}{$\bar{j}$} & \multirow[t]{2}{*}{$g f$} & \multirow{2}{*}{$\begin{array}{c}A \\
\mathrm{~s}^{-1}\end{array}$} & \multicolumn{7}{|c|}{ Effective Collision strength $\Upsilon$} \\
\hline & & & & 0.5 & 1.0 & 2.0 & 3.0 & 4.0 & 5.0 & 6.0 \\
\hline 1 & 62 & $1.716-02$ & $2.929+10$ & $1.503-03$ & $1.628-03$ & $1.877-03$ & $2.110-03$ & $2.328-03$ & $2.530-03$ & $2.720-03$ \\
\hline 1 & 63 & $1.057-04$ & $1.841+08$ & $3.384-05$ & $3.166-05$ & $2.911-05$ & $2.780-05$ & $2.713-05$ & $2.684-05$ & $2.678-05$ \\
\hline 1 & 64 & $4.122-06$ & $3.596+06$ & $3.933-05$ & $3.528-05$ & $2.961-05$ & $2.575-05$ & $2.291-05$ & $2.072-05$ & $1.897-05$ \\
\hline 1 & 65 & & & $2.431-05$ & $2.164-05$ & $1.796-05$ & $1.548-05$ & $1.368-05$ & $1.229-05$ & $1.118-05$ \\
\hline 1 & 66 & & & $5.658-03$ & $4.937-03$ & $3.998-03$ & $3.397-03$ & 2.971-03 & $2.651-03$ & $2.399-03$ \\
\hline 1 & 67 & $8.161-05$ & $4.831+07$ & $1.212-02$ & $1.301-02$ & $1.450-02$ & $1.569-02$ & $1.668-02$ & $1.752-02$ & $1.824-02$ \\
\hline 1 & 68 & 7.911-13 & $4.992+07$ & $8.147-03$ & $8.767-03$ & $9.791-03$ & $1.061-02$ & 1.129-02 & $1.186-02$ & $1.236-02$ \\
\hline 1 & 69 & $3.772-05$ & $2.257+07$ & 7.064-03 & $7.289-03$ & $7.726-03$ & 8.113-03 & 8.453-03 & $8.751-03$ & $9.016-03$ \\
\hline 1 & 70 & $1.045-02$ & $1.895+10$ & 3.395-04 & $4.124-04$ & 5.569-04 & 6.932-04 & $8.206-04$ & 9.396-04 & $1.051-03$ \\
\hline 1 & 71 & $4.526-13$ & $8.222-01$ & $1.301-03$ & $1.170-03$ & $9.967-04$ & $8.853-04$ & $8.060-04$ & $7.460-04$ & 6.989-04 \\
\hline 1 & 72 & $6.206-13$ & $3.436+07$ & 6.209-03 & $6.561-03$ & $7.167-03$ & 7.666-03 & 8.086-03 & 8.446-03 & $8.760-03$ \\
\hline 1 & 73 & $6.200-03$ & $5.640+09$ & $2.552-04$ & $2.925-04$ & $3.698-04$ & $4.451-04$ & $5.166-04$ & $5.842-04$ & $6.481-04$ \\
\hline 1 & 74 & & & 3.819-05 & $3.347-05$ & 2.719-05 & $2.312-05$ & $2.023-05$ & $1.804-05$ & $1.633-05$ \\
\hline 1 & 75 & $1.455-12$ & $2.683+00$ & $1.091-04$ & $9.716-05$ & $8.080-05$ & 6.985-05 & $6.188-05$ & 5.575-05 & 5.087-05 \\
\hline 1 & 76 & $3.090-12$ & $2.192+04$ & $1.868-04$ & $1.669-04$ & $1.396-04$ & $1.214-04$ & $1.081-04$ & $9.796-05$ & 8.986-05 \\
\hline 1 & 77 & 5.823-09 & $3.589+03$ & $1.984-04$ & $1.768-04$ & $1.471-04$ & $1.272-04$ & $1.126-04$ & $1.014-04$ & $9.250-05$ \\
\hline 1 & 78 & $8.523-11$ & $5.262+01$ & $5.502-05$ & 4.864-05 & $4.006-05$ & $3.443-05$ & $3.037-05$ & $2.728-05$ & $2.484-05$ \\
\hline 1 & 79 & $2.703-03$ & $2.504+09$ & $9.920-05$ & $1.133-04$ & $1.438-04$ & $1.742-04$ & $2.033-04$ & $2.310-04$ & $2.573-04$ \\
\hline 1 & 80 & & & $1.623-04$ & $1.448-04$ & $1.207-04$ & $1.044-04$ & $9.245-05$ & 8.324-05 & $7.588-05$ \\
\hline 1 & 81 & $2.037-12$ & $3.790+00$ & 5.894-05 & $5.269-05$ & $4.402-05$ & $3.817-05$ & 3.389-05 & $3.059-05$ & $2.794-05$ \\
\hline 1 & 82 & 3.899-13 & $7.983+03$ & $1.180-04$ & $1.057-04$ & $8.890-05$ & 7.763-05 & $6.943-05$ & $6.312-05$ & 5.809-05 \\
\hline 1 & 83 & $4.488-12$ & $2.791+00$ & $1.498-04$ & $1.335-04$ & $1.111-04$ & $9.606-05$ & $8.507-05$ & $7.661-05$ & $6.986-05$ \\
\hline 1 & 84 & $4.634-13$ & $1.668+04$ & $1.113-04$ & $1.002-04$ & 8.604-05 & $7.722-05$ & 7.119-05 & $6.683-05$ & $6.354-05$ \\
\hline 1 & 85 & $1.253-08$ & $7.885+03$ & $1.028-04$ & $9.591-05$ & $8.720-05$ & $8.201-05$ & 7.859-05 & $7.621-05$ & $7.448-05$ \\
\hline 1 & 86 & $1.050-12$ & $5.319+04$ & $8.988-05$ & $8.900-05$ & 8.919-05 & $9.039-05$ & $9.189-05$ & $9.345-05$ & $9.496-05$ \\
\hline 1 & 87 & $7.027-13$ & $1.345+00$ & $4.083-04$ & $4.152-04$ & $4.249-04$ & $4.320-04$ & 4.375-04 & $4.419-04$ & $4.457-04$ \\
\hline 1 & 88 & $5.699-13$ & $1.528+03$ & $5.715-05$ & $5.020-05$ & $4.093-05$ & $3.493-05$ & $3.067-05$ & $2.747-05$ & $2.495-05$ \\
\hline 1 & 89 & $\begin{array}{l}6.689-05 \\
\text { available }\end{array}$ & $\begin{array}{l}6.487+07 \\
\text { request }\end{array}$ & $1.738-04$ & $1.512-04$ & $1.225-04$ & $1.048-04$ & $9.252-05$ & $8.353-05$ & 7.663-05 \\
\hline 15 & 161 & $1.274-13$ & $3.859+04$ & $1.866-04$ & $1.801-04$ & $1.730-04$ & $1.704-04$ & $1.687-04$ & $1.671-04$ & $1.659-04$ \\
\hline 15 & 162 & $4.972-15$ & $4.941+01$ & $9.193-05$ & $8.048-05$ & $6.526-05$ & 5.589-05 & $4.830-05$ & $4.152-05$ & $3.583-05$ \\
\hline 15 & 163 & $9.447-14$ & $1.253+02$ & 4.969-05 & $4.341-05$ & $3.508-05$ & 2.995-05 & $2.580-05$ & $2.209-05$ & $1.898-05$ \\
\hline 15 & 164 & 2.907-04 & $1.369+08$ & $5.688-05$ & $5.860-05$ & $6.276-05$ & $6.736-05$ & 7.178-05 & $7.590-05$ & 7.974-05 \\
\hline 15 & 165 & $3.556-12$ & $1.256+00$ & $3.263-05$ & $2.802-05$ & $2.215-05$ & $1.872-05$ & $1.598-05$ & $1.353-05$ & $1.148-05$ \\
\hline 15 & 166 & $7.110-05$ & $5.029+07$ & $4.268-05$ & $3.987-05$ & $3.688-05$ & $3.574-05$ & $3.503-05$ & $3.441-05$ & $3.398-05$ \\
\hline 15 & 167 & & & $1.203-04$ & $1.074-04$ & $9.135-05$ & 8.233-05 & $7.522-05$ & $6.879-05$ & 6.339-05 \\
\hline 15 & 168 & $2.850-06$ & $1.345+06$ & 7.940-05 & 7.941-05 & $8.061-05$ & $8.250-05$ & $8.427-05$ & $8.575-05$ & $8.698-05$ \\
\hline 15 & 169 & 6.709-12 & $2.376+00$ & $1.329-04$ & $1.398-04$ & $1.514-04$ & $1.609-04$ & $1.692-04$ & $1.764-04$ & $1.825-04$ \\
\hline 15 & 170 & $3.612-11$ & $1.281+01$ & $2.462-04$ & 2.618-04 & $2.878-04$ & $3.096-04$ & $3.286-04$ & $3.453-04$ & $3.596-04$ \\
\hline 15 & 171 & 2.194-03 & $1.038+09$ & $6.513-05$ & 5.559-05 & $4.376-05$ & $3.698-05$ & $3.161-05$ & $2.679-05$ & $2.276-05$ \\
\hline 15 & 172 & $3.921-15$ & $1.819+05$ & $3.925-04$ & $4.133-04$ & $4.549-04$ & $4.958-04$ & 5.343-04 & $5.698-04$ & $6.026-04$ \\
\hline 15 & 173 & & & $4.974-04$ & $5.249-04$ & $5.760-04$ & $6.232-04$ & $6.658-04$ & $7.038-04$ & $7.372-04$ \\
\hline 15 & 174 & & & $7.257-04$ & 7.826-04 & $8.757-04$ & $9.521-04$ & $1.019-03$ & $1.078-03$ & $1.128-03$ \\
\hline 15 & 175 & $1.486-11$ & $5.287+00$ & $1.722-04$ & $1.466-04$ & $1.149-04$ & $9.650-05$ & $8.194-05$ & $6.891-05$ & $5.804-05$ \\
\hline 15 & 176 & $1.696-06$ & $6.039+05$ & $2.237-04$ & $2.118-04$ & $1.985-04$ & $1.927-04$ & $1.885-04$ & $1.845-04$ & $1.811-04$ \\
\hline 15 & 177 & $1.687-14$ & $4.689+05$ & $1.441-04$ & $1.206-04$ & $9.205-05$ & $7.582-05$ & $6.303-05$ & $5.156-05$ & $4.202-05$ \\
\hline 15 & 178 & $1.468-10$ & $5.230+01$ & $2.053-04$ & $2.195-04$ & $2.435-04$ & 2.639-04 & 2.819-04 & $2.978-04$ & $3.115-04$ \\
\hline 15 & 179 & $1.716-03$ & $8.149+08$ & $3.446-04$ & $3.546-04$ & $3.726-04$ & $3.887-04$ & $4.027-04$ & $4.146-04$ & $4.245-04$ \\
\hline 15 & 180 & & & 3.763-04 & $3.763-04$ & $3.868-04$ & $4.043-04$ & $4.221-04$ & $4.389-04$ & $4.550-04$ \\
\hline 15 & 181 & $6.370-05$ & $4.543+07$ & $3.129-04$ & $3.430-04$ & $3.867-04$ & $4.183-04$ & $4.446-04$ & $4.672-04$ & $4.856-04$ \\
\hline 15 & 182 & $2.705-13$ & $8.756+05$ & $1.162-04$ & $1.021-04$ & $8.480-05$ & $7.521-05$ & $6.775-05$ & 6.109-05 & $5.560-05$ \\
\hline 15 & 183 & 3.799-09 & $5.423+03$ & $5.257-05$ & $4.461-05$ & $3.464-05$ & $2.884-05$ & $2.423-05$ & $2.011-05$ & $1.667-05$ \\
\hline 15 & 184 & $1.514-02$ & $7.209+09$ & $2.198-03$ & $2.380-03$ & $2.703-03$ & 2.994-03 & $3.262-03$ & $3.508-03$ & $3.733-03$ \\
\hline 15 & 185 & $2.391-03$ & $1.710+09$ & 5.564-04 & $5.952-04$ & $6.616-04$ & 7.196-04 & $7.720-04$ & $8.196-04$ & $8.621-04$ \\
\hline 15 & 186 & $9.921-04$ & $1.500+09$ & 7.649-05 & $8.162-05$ & $9.268-05$ & $1.041-04$ & $1.150-04$ & $1.254-04$ & $1.352-04$ \\
\hline 15 & 187 & $8.517-13$ & $3.141+05$ & $1.011-04$ & $9.326-05$ & $8.318-05$ & $7.755-05$ & 7.316-05 & 6.924-05 & $6.602-05$ \\
\hline 15 & 188 & $1.382-12$ & $1.571+05$ & $1.509-03$ & $1.519-03$ & $1.538-03$ & $1.559-03$ & $1.577-03$ & $1.593-03$ & $1.605-03$ \\
\hline 15 & 189 & $5.450-13$ & $8.359+02$ & $3.753-04$ & $3.583-04$ & $3.427-04$ & $3.402-04$ & $3.405-04$ & $3.409-04$ & $3.422-04$ \\
\hline 15 & 190 & $1.656-15$ & $3.359+01$ & 5.816-05 & $5.133-05$ & $4.222-05$ & $3.671-05$ & $3.230-05$ & $2.839-05$ & $2.515-05$ \\
\hline 15 & 191 & $5.610-04$ & $4.369+08$ & $1.571-03$ & $1.642-03$ & $1.779-03$ & $1.913-03$ & $2.036-03$ & $2.150-03$ & $2.253-03$ \\
\hline
\end{tabular}


Table XI-continued ...

\begin{tabular}{|c|c|c|c|c|c|c|c|c|c|c|}
\hline \multirow[t]{2}{*}{ i } & \multirow[t]{2}{*}{$\mathrm{j}$} & \multirow[t]{2}{*}{$g f$} & \multirow{2}{*}{$\begin{array}{c}A \\
\mathrm{~s}^{-1}\end{array}$} & \multicolumn{7}{|c|}{ Effective Collision strength $\Upsilon$} \\
\hline & & & & 0.5 & 1.0 & 2.0 & 3.0 & 4.0 & 5.0 & 6.0 \\
\hline 15 & 192 & $5.099-03$ & $2.648+09$ & $8.745-04$ & $8.812-04$ & $8.932-04$ & $9.065-04$ & $9.184-04$ & $9.282-04$ & $9.361-04$ \\
\hline 15 & 193 & $2.885-14$ & $1.129-02$ & $3.018-05$ & $2.606-05$ & $2.139-05$ & $1.900-05$ & $1.717-05$ & $1.551-05$ & $1.413-05$ \\
\hline 15 & 194 & & & $4.483-05$ & $4.209-05$ & $3.951-05$ & $3.865-05$ & $3.811-05$ & $3.756-05$ & $3.710-05$ \\
\hline 15 & 195 & $5.899-14$ & $8.899+04$ & $1.416-04$ & $1.258-04$ & $1.084-04$ & $1.003-04$ & $9.441-05$ & 8.899-05 & $8.452-05$ \\
\hline 15 & 196 & $1.043-06$ & $4.093+05$ & $2.662-04$ & $2.616-04$ & $2.636-04$ & $2.719-04$ & $2.805-04$ & $2.877-04$ & 2.941-04 \\
\hline 15 & 197 & $3.665-04$ & $2.930+08$ & $7.151-05$ & $6.759-05$ & $6.392-05$ & $6.328-05$ & $6.335-05$ & $6.357-05$ & $6.411-05$ \\
\hline 15 & 198 & $1.792-03$ & $9.556+08$ & $1.173-04$ & $1.165-04$ & $1.204-04$ & $1.282-04$ & $1.366-04$ & $1.450-04$ & $1.534-04$ \\
\hline 15 & 199 & $9.215-04$ & $1.475+09$ & $9.884-05$ & $1.073-04$ & $1.244-04$ & $1.410-04$ & $1.566-04$ & $1.709-04$ & $1.840-04$ \\
\hline 15 & 200 & $3.423-03$ & $2.742+09$ & $3.116-04$ & $3.600-04$ & $4.486-04$ & $5.281-04$ & 6.007-04 & $6.670-04$ & $7.267-04$ \\
\hline 15 & 201 & $3.988-03$ & $6.439+09$ & $2.655-04$ & $2.832-04$ & $3.200-04$ & $3.575-04$ & $3.938-04$ & $4.283-04$ & $4.613-04$ \\
\hline 15 & 202 & $5.295-14$ & $5.356+04$ & 9.944-05 & $1.006-04$ & $1.035-04$ & $1.067-04$ & $1.098-04$ & $1.124-04$ & $1.147-04$ \\
\hline 15 & 203 & $1.549-13$ & $1.046+05$ & 2.095-04 & $2.178-04$ & $2.320-04$ & $2.445-04$ & $2.556-04$ & 2.653-04 & $2.736-04$ \\
\hline 15 & 204 & $6.120-07$ & $2.502+05$ & $2.434-04$ & $2.456-04$ & $2.524-04$ & 2.609-04 & 2.689-04 & $2.759-04$ & 2.821-04 \\
\hline 15 & 205 & $1.890-14$ & $1.890+04$ & $1.070-04$ & $9.653-05$ & $8.285-05$ & 7.473-05 & 6.824-05 & $6.245-05$ & $5.760-05$ \\
\hline 15 & 206 & $4.130-14$ & $4.149+05$ & $5.671-05$ & $5.849-05$ & $6.235-05$ & $6.630-05$ & 6.996-05 & 7.323-05 & 7.613-05 \\
\hline 15 & 207 & $1.284-13$ & $1.856+05$ & $2.247-04$ & $2.320-04$ & $2.498-04$ & 2.695-04 & 2.881-04 & $3.050-04$ & $3.203-04$ \\
\hline 15 & 208 & $2.820-05$ & $1.552+07$ & $6.750-05$ & $6.487-05$ & $6.262-05$ & $6.234-05$ & $6.237-05$ & $6.232-05$ & $6.232-05$ \\
\hline 15 & 209 & $5.008-11$ & $2.067+01$ & $8.833-05$ & $8.605-05$ & $8.470-05$ & $8.538-05$ & $8.625-05$ & $8.690-05$ & $8.744-05$ \\
\hline 15 & 210 & $5.308-13$ & 2.203-01 & $1.973-04$ & $1.789-04$ & $1.565-04$ & $1.442-04$ & $1.346-04$ & $1.259-04$ & $1.186-04$ \\
\hline 15 & 211 & & & 3.093-04 & $3.093-04$ & $3.130-04$ & $3.189-04$ & $3.245-04$ & $3.290-04$ & $3.327-04$ \\
\hline 15 & 212 & $9.007-04$ & $7.492+08$ & $2.114-04$ & $2.041-04$ & $1.989-04$ & $2.005-04$ & $2.035-04$ & $2.065-04$ & $2.100-04$ \\
\hline 15 & 213 & 6.849-03 & $3.798+09$ & 8.831-04 & $9.206-04$ & $9.992-04$ & $1.080-03$ & $1.157-03$ & $1.230-03$ & $1.297-03$ \\
\hline 15 & 219 & 7.324-06 & $1.272+07$ & $1.361-05$ & $1.297-05$ & $1.234-05$ & $1.217-05$ & $1.211-05$ & $1.207-05$ & $1.207-05$ \\
\hline 15 & 228 & $4.294-13$ & $1.409+06$ & $3.778-05$ & $3.405-05$ & $2.910-05$ & $2.612-05$ & $2.373-05$ & $2.160-05$ & $1.982-05$ \\
\hline 15 & 229 & $2.413-13$ & $1.077+06$ & $2.125-04$ & $2.069-04$ & $1.994-04$ & $1.955-04$ & $1.925-04$ & $1.896-04$ & $1.872-04$ \\
\hline 15 & 236 & $4.907-04$ & $4.324+08$ & $1.310-04$ & $1.235-04$ & $1.157-04$ & $1.132-04$ & $1.117-04$ & $1.105-04$ & $1.097-04$ \\
\hline 15 & 237 & 7.034-03 & $4.133+09$ & $5.020-04$ & 5.194-04 & $5.555-04$ & 5.921-04 & $6.263-04$ & $6.574-04$ & $6.856-04$ \\
\hline 15 & 242 & & & $3.631-07$ & $2.827-07$ & $1.998-07$ & $1.613-07$ & $1.329-07$ & $1.063-07$ & $8.405-08$ \\
\hline 15 & 243 & & & 4.479-07 & $3.526-07$ & $2.551-07$ & $2.111-07$ & $1.802-07$ & $1.527-07$ & $1.311-07$ \\
\hline 15 & 247 & $2.965-13$ & $5.022+04$ & $4.932-05$ & $4.302-05$ & $3.601-05$ & $3.267-05$ & $3.021-05$ & $2.795-05$ & $2.610-05$ \\
\hline 15 & 248 & $1.392-08$ & $6.156+03$ & $8.287-05$ & 7.895-05 & $7.646-05$ & $7.721-05$ & $7.840-05$ & 7.936-05 & $8.028-05$ \\
\hline 15 & 249 & $7.788-15$ & $3.446-03$ & $5.359-06$ & $4.285-06$ & $3.110-06$ & $2.527-06$ & $2.086-06$ & $1.682-06$ & $1.345-06$ \\
\hline 15 & 250 & & & $6.712-06$ & 5.396-06 & $3.962-06$ & $3.249-06$ & $2.711-06$ & 2.219-06 & $1.809-06$ \\
\hline
\end{tabular}


TABLE XII:

\begin{tabular}{|c|c|c|c|c|c|c|c|c|c|c|}
\hline \multirow[t]{2}{*}{$\mathrm{i}$} & \multirow[t]{2}{*}{$\mathrm{j}$} & \multirow{2}{*}{$\overline{g f f}$} & \multirow{2}{*}{$\begin{array}{c}A \\
\mathrm{~s}^{-1}\end{array}$} & \multicolumn{7}{|c|}{ 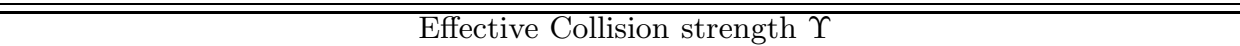 } \\
\hline & & & & 0.5 & 1.0 & 2.0 & 3.0 & 4.0 & 5.0 & 6.0 \\
\hline 1 & 2 & & & $5.049-03$ & $4.672-03$ & $4.154-03$ & $3.669-03$ & $3.192-03$ & $2.821-03$ & $2.525-03$ \\
\hline 1 & 3 & $4.835-05$ & $3.173+05$ & $1.558-02$ & $1.447-02$ & $1.295-02$ & 1.154-02 & $1.015-02$ & $9.068-03$ & 8.208-03 \\
\hline 1 & 4 & $5.669-11$ & $2.367-01$ & $2.505-02$ & $2.318-02$ & $2.061-02$ & $1.820-02$ & $1.584-02$ & $1.400-02$ & $1.253-02$ \\
\hline 1 & 5 & $2.666-01$ & $6.641+09$ & $1.103+00$ & $1.140+00$ & $1.219+00$ & $1.295+00$ & $1.373+00$ & $1.438+00$ & $1.493+00$ \\
\hline 1 & 6 & & & $2.327-04$ & $2.131-04$ & $1.877-04$ & $1.631-04$ & $1.355-04$ & $1.160-04$ & $1.008-04$ \\
\hline 1 & 7 & $4.846-10$ & $2.139+01$ & $6.676-04$ & $6.098-04$ & $5.347-04$ & $4.620-04$ & $3.804-04$ & $3.227-04$ & $2.776-04$ \\
\hline 1 & 8 & $6.542-10$ & $1.767+01$ & $1.123-03$ & $1.031-03$ & $9.108-04$ & 7.951-04 & $6.650-04$ & $5.733-04$ & 5.018-04 \\
\hline 1 & 9 & $1.497-07$ & $4.958+03$ & $6.800-03$ & $6.963-03$ & 7.319-03 & $7.662-03$ & $8.028-03$ & $8.307-03$ & 8.536-03 \\
\hline 1 & 10 & & & $2.180-03$ & $2.146-03$ & $2.117-03$ & 2.091-03 & $2.070-03$ & $2.051-03$ & $2.036-03$ \\
\hline 1 & 11 & $7.746-11$ & $8.391+01$ & 4.116-03 & $3.779-03$ & $3.344-03$ & $2.922-03$ & $2.432-03$ & $2.090-03$ & $1.822-03$ \\
\hline 1 & 12 & & & $5.331-02$ & $5.378-02$ & $5.515-02$ & $5.648-02$ & $5.801-02$ & $5.912-02$ & $6.001-02$ \\
\hline 1 & 13 & 5.533-01 & $6.425+11$ & 2.371-02 & $2.799-02$ & $3.680-02$ & $4.518-02$ & $5.285-02$ & $6.005-02$ & $6.662-02$ \\
\hline 1 & 14 & & & $9.236-04$ & $8.489-04$ & $7.503-04$ & $6.559-04$ & $5.528-04$ & $4.780-04$ & $4.188-04$ \\
\hline 1 & 15 & $3.592-02$ & $4.185+10$ & $3.975-03$ & $4.025-03$ & $4.279-03$ & 4.519-03 & $4.692-03$ & $4.916-03$ & 5.146-03 \\
\hline 1 & 16 & $2.424-08$ & $1.696+04$ & $4.575-03$ & $4.205-03$ & $3.718-03$ & $3.251-03$ & $2.741-03$ & $2.370-03$ & $2.077-03$ \\
\hline 1 & 17 & $6.595-12$ & $7.969+00$ & $5.270-03$ & $4.827-03$ & $4.256-03$ & $3.703-03$ & $3.074-03$ & $2.631-03$ & $2.283-03$ \\
\hline 1 & 18 & $3.219-08$ & $2.334+04$ & 8.802-03 & $8.062-03$ & $7.106-03$ & $6.178-03$ & $5.130-03$ & $4.392-03$ & 3.813-03 \\
\hline 1 & 19 & & & $1.229-02$ & $1.126-02$ & 9.931-03 & 8.643-03 & 7.173-03 & 6.141-03 & $5.327-03$ \\
\hline 1 & 20 & $4.552-04$ & $3.394+08$ & $6.490-02$ & $6.889-02$ & $7.688-02$ & 8.438-02 & $9.160-02$ & $9.758-02$ & $1.026-01$ \\
\hline 1 & 21 & & & $1.457-05$ & $1.342-05$ & $1.191-05$ & $1.045-05$ & $8.915-06$ & $7.777-06$ & $6.875-06$ \\
\hline 1 & 22 & $3.744-04$ & $4.906+08$ & 5.604-05 & $5.510-05$ & $5.564-05$ & $5.615-05$ & $5.598-05$ & $5.678-05$ & $5.795-05$ \\
\hline 1 & 23 & $1.356-11$ & $1.071+01$ & $6.990-05$ & $6.441-05$ & $5.710-05$ & $5.015-05$ & $4.278-05$ & $3.733-05$ & $3.300-05$ \\
\hline 1 & 24 & $2.114-02$ & $2.855+10$ & 7.324-04 & $8.630-04$ & $1.140-03$ & $1.403-03$ & $1.642-03$ & $1.871-03$ & $2.083-03$ \\
\hline 1 & 25 & $3.663-12$ & $4.984+00$ & $8.313-05$ & 7.634-05 & $6.723-05$ & $5.854-05$ & $4.891-05$ & $4.202-05$ & $3.659-05$ \\
\hline 1 & 26 & $3.497-14$ & $4.792-02$ & $1.787-04$ & $1.648-04$ & 1.461-04 & $1.282-04$ & $1.092-04$ & $9.525-05$ & 8.415-05 \\
\hline 1 & 27 & 4.418-09 & $3.638+03$ & $3.215-04$ & $2.968-04$ & $2.636-04$ & $2.318-04$ & $1.981-04$ & $1.732-04$ & $1.535-04$ \\
\hline 1 & 28 & & & $4.280-04$ & $3.950-04$ & $3.501-04$ & $3.077-04$ & $2.625-04$ & $2.291-04$ & $2.026-04$ \\
\hline 1 & 29 & $3.811-14$ & $5.307-02$ & $7.928-05$ & 7.371-05 & $6.593-05$ & $5.854-05$ & $5.084-05$ & $4.503-05$ & $4.038-05$ \\
\hline 1 & 30 & & & $2.298-05$ & $2.126-05$ & $1.904-05$ & $1.690-05$ & $1.449-05$ & $1.279-05$ & $1.146-05$ \\
\hline 1 & 31 & $8.069-12$ & $1.135+01$ & 5.983-05 & $5.476-05$ & $4.812-05$ & $4.173-05$ & $3.453-05$ & $2.943-05$ & $2.544-05$ \\
\hline 1 & 32 & $1.948-08$ & $1.647+04$ & $1.014-04$ & $9.356-05$ & $8.338-05$ & $7.355-05$ & $6.234-05$ & $5.453-05$ & $4.843-05$ \\
\hline 1 & 33 & $3.033-10$ & $2.571+02$ & $2.176-04$ & $1.984-04$ & $1.738-04$ & $1.503-04$ & $1.224-04$ & $1.032-04$ & 8.823-05 \\
\hline 1 & 34 & & & 3.371-04 & $3.079-04$ & $2.709-04$ & $2.347-04$ & $1.923-04$ & $1.630-04$ & $1.401-04$ \\
\hline 1 & 35 & 1.070-09 & $9.124+02$ & $1.253-04$ & $1.137-04$ & $9.945-05$ & $8.548-05$ & $6.837-05$ & $5.690-05$ & 4.796-05 \\
\hline 1 & 36 & & & 4.234-04 & 3.861-04 & $3.390-04$ & 2.931-04 & $2.395-04$ & $2.025-04$ & $1.736-04$ \\
\hline 1 & 37 & $1.227-06$ & $1.057+06$ & $3.434-04$ & $3.672-04$ & $4.152-04$ & $4.603-04$ & $5.021-04$ & $5.381-04$ & 5.688-04 \\
\hline 1 & 38 & 3.029-04 & $4.375+08$ & $9.524-05$ & $9.203-05$ & $9.001-05$ & $8.790-05$ & 8.393-05 & $8.236-05$ & 8.163-05 \\
\hline 1 & 39 & $2.288-10$ & $1.985+02$ & $1.145-04$ & $1.040-04$ & $9.102-05$ & $7.822-05$ & $6.266-05$ & $5.221-05$ & $4.407-05$ \\
\hline 1 & 40 & & & $1.279-04$ & $1.160-04$ & $1.016-04$ & $8.737-05$ & $6.944-05$ & $5.767-05$ & $4.851-05$ \\
\hline 1 & 41 & 1.874-09 & $1.639+03$ & $3.240-04$ & 2.973-04 & $2.619-04$ & $2.282-04$ & $1.906-04$ & $1.638-04$ & $1.427-04$ \\
\hline 1 & 42 & $6.010-05$ & $8.768+07$ & $2.052-04$ & $1.892-04$ & $1.687-04$ & $1.491-04$ & $1.271-04$ & $1.115-04$ & $9.940-05$ \\
\hline 1 & 43 & & & $6.783-05$ & $6.214-05$ & $5.479-05$ & $4.776-05$ & 3.994-05 & $3.434-05$ & 2.994-05 \\
\hline 1 & 44 & & & $5.138-04$ & $5.134-04$ & $5.185-04$ & $5.237-04$ & $5.307-04$ & $5.353-04$ & 5.391-04 \\
\hline 1 & 45 & & & $5.110-04$ & 5.471-04 & $6.100-04$ & 6.691-04 & $7.273-04$ & 7.739-04 & $8.120-04$ \\
\hline 1 & 46 & 2.973-02 & $4.452+10$ & $3.189-03$ & $3.426-03$ & $3.921-03$ & $4.394-03$ & $4.844-03$ & $5.248-03$ & 5.611-03 \\
\hline 1 & 47 & $3.319-11$ & $6.415+01$ & 1.304-03 & $1.196-03$ & $1.059-03$ & $9.251-04$ & $7.692-04$ & $6.606-04$ & $5.750-04$ \\
\hline 1 & 48 & & & $9.938-03$ & $1.007-02$ & $1.040-02$ & $1.071-02$ & $1.106-02$ & $1.131-02$ & 1.153-02 \\
\hline 1 & 49 & & & $3.466-04$ & $3.189-04$ & $2.820-04$ & $2.468-04$ & $2.082-04$ & $1.803-04$ & $1.582-04$ \\
\hline 1 & 50 & $2.122-03$ & $4.190+09$ & $1.100-03$ & $1.030-03$ & $9.460-04$ & 8.659-04 & $7.742-04$ & $7.122-04$ & $6.657-04$ \\
\hline 1 & 51 & $1.068-08$ & $1.266+04$ & $1.716-03$ & $1.579-03$ & $1.397-03$ & $1.222-03$ & $1.032-03$ & 8.934-04 & 7.839-04 \\
\hline 1 & 52 & $1.613-01$ & $3.192+11$ & $5.407-03$ & 6.333-03 & $8.244-03$ & $1.006-02$ & $1.173-02$ & $1.330-02$ & 1.473-02 \\
\hline 1 & 53 & $2.383-12$ & $4.758+00$ & $1.551-03$ & $1.423-03$ & $1.256-03$ & $1.097-03$ & $9.232-04$ & 7.973-04 & 6.984-04 \\
\hline 1 & 54 & 6.647-09 & $7.964+03$ & 2.583-03 & $2.372-03$ & $2.096-03$ & $1.830-03$ & $1.540-03$ & $1.331-03$ & 1.166-03 \\
\hline 1 & 55 & & & $3.612-03$ & $3.317-03$ & $2.928-03$ & $2.557-03$ & $2.152-03$ & $1.858-03$ & $1.628-03$ \\
\hline 1 & 56 & $6.135-05$ & $7.403+07$ & $9.772-04$ & 8.895-04 & $7.810-04$ & $6.725-04$ & $5.334-04$ & $4.426-04$ & $3.716-04$ \\
\hline 1 & 57 & $1.891-16$ & $2.282-04$ & $1.351-03$ & $1.235-03$ & $1.088-03$ & $9.397-04$ & 7.464-04 & $6.210-04$ & $5.225-04$ \\
\hline 1 & 58 & & & $1.758-03$ & $1.600-03$ & $1.404-03$ & $1.210-03$ & $9.596-04$ & 7.961-04 & $6.685-04$ \\
\hline 1 & 59 & & & $1.133-02$ & $1.199-02$ & $1.332-02$ & $1.458-02$ & $1.578-02$ & $1.679-02$ & 1.764-02 \\
\hline 1 & 60 & & & $3.148-03$ & $3.416-03$ & $3.894-03$ & $4.342-03$ & $4.775-03$ & $5.122-03$ & 5.404-03 \\
\hline 1 & 61 & & & $5.142-06$ & $4.748-06$ & $4.216-06$ & $3.711-06$ & $3.194-06$ & $2.803-06$ & $2.492-06$ \\
\hline
\end{tabular}


Table XII-continued ...

\begin{tabular}{|c|c|c|c|c|c|c|c|c|c|c|}
\hline \multirow[t]{2}{*}{ i } & \multirow[t]{2}{*}{$\mathrm{j}$} & \multirow[t]{2}{*}{$g f$} & \multirow{2}{*}{$\begin{array}{c}A \\
\mathrm{~s}^{-1}\end{array}$} & \multicolumn{7}{|c|}{ 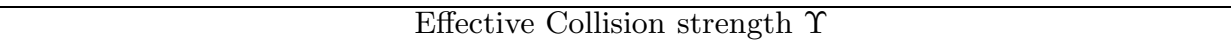 } \\
\hline & & & & 0.5 & 1.0 & 2.0 & 3.0 & 4.0 & 5.0 & 6.0 \\
\hline 1 & 62 & $1.001-07$ & $2.212+05$ & $1.521-05$ & $1.407-05$ & $1.253-05$ & $1.108-05$ & $9.588-06$ & $8.462-06$ & $7.570-06$ \\
\hline 1 & 63 & 7.161-12 & $9.531+00$ & $2.490-05$ & $2.300-05$ & $2.041-05$ & $1.798-05$ & $1.549-05$ & $1.360-05$ & $1.210-05$ \\
\hline 1 & 64 & $3.017-05$ & $6.720+07$ & $1.085-05$ & $1.008-05$ & $9.077-06$ & 8.119-06 & $7.088-06$ & $6.346-06$ & $5.769-06$ \\
\hline 1 & 65 & 8.399-13 & $1.880+00$ & $3.332-05$ & $3.065-05$ & $2.709-05$ & 2.369-05 & $1.996-05$ & $1.726-05$ & $1.513-05$ \\
\hline 1 & 66 & $4.697-13$ & $1.054+00$ & $3.434-05$ & $3.161-05$ & $2.796-05$ & $2.447-05$ & $2.066-05$ & $1.790-05$ & $1.572-05$ \\
\hline 1 & 67 & 1.427-09 & $1.922+03$ & $6.530-05$ & $6.022-05$ & $5.345-05$ & 4.698-05 & $3.997-05$ & $3.485-05$ & $3.081-05$ \\
\hline 1 & 68 & & & $8.971-05$ & $8.265-05$ & $7.326-05$ & $6.428-05$ & $5.458-05$ & $4.749-05$ & $4.187-05$ \\
\hline 1 & 69 & $1.484-13$ & $3.347-01$ & $5.298-05$ & $4.896-05$ & $4.353-05$ & $3.833-05$ & $3.279-05$ & 2.869-05 & 2.544-05 \\
\hline 1 & 70 & & & $2.087-05$ & 2.039-05 & $1.995-05$ & $1.953-05$ & $1.904-05$ & $1.871-05$ & $1.846-05$ \\
\hline 1 & 71 & 2.956-12 & $6.688+00$ & 7.381-05 & $6.734-05$ & 5.914-05 & $5.116-05$ & 4.196-05 & $3.556-05$ & $3.055-05$ \\
\hline 1 & 72 & $8.239-11$ & $1.119+02$ & $3.259-05$ & $3.001-05$ & $2.653-05$ & 2.319-05 & $1.954-05$ & $1.690-05$ & $1.482-05$ \\
\hline 1 & 73 & $2.563-10$ & $3.482+02$ & $3.685-05$ & $3.382-05$ & 2.981-05 & 2.597-05 & $2.161-05$ & $1.855-05$ & $1.616-05$ \\
\hline 1 & 74 & & & $1.129-04$ & $1.035-04$ & $9.166-05$ & 8.004-05 & 6.669-05 & $5.741-05$ & $5.015-05$ \\
\hline 1 & 75 & $4.944-11$ & $6.734+01$ & $5.647-05$ & $5.142-05$ & 4.507-05 & 3.889-05 & $3.159-05$ & 2.659-05 & $2.268-05$ \\
\hline 1 & 76 & & & $1.465-04$ & $1.338-04$ & $1.176-04$ & $1.019-04$ & 8.397-05 & 7.139-05 & $6.154-05$ \\
\hline 1 & 77 & $1.220-09$ & $1.666+03$ & $1.310-05$ & $1.303-05$ & $1.387-05$ & $1.455-05$ & $1.454-05$ & $1.506-05$ & $1.566-05$ \\
\hline 1 & 78 & 5.829-04 & $1.327+09$ & 7.609-05 & 7.693-05 & $8.087-05$ & 8.456-05 & $8.730-05$ & $9.062-05$ & 9.394-05 \\
\hline 1 & 79 & & & $1.984-05$ & $1.802-05$ & $1.580-05$ & $1.355-05$ & $1.047-05$ & $8.532-06$ & 7.022-06 \\
\hline 1 & 80 & $6.688-10$ & $9.148+02$ & $1.504-05$ & 1.361-05 & 1.191-05 & $1.018-05$ & 7.829-06 & $6.348-06$ & 5.199-06 \\
\hline 1 & 81 & & & $2.468-05$ & $2.319-05$ & $2.183-05$ & $2.032-05$ & $1.788-05$ & $1.652-05$ & $1.549-05$ \\
\hline 1 & 82 & & & $6.624-05$ & $6.051-05$ & $5.311-05$ & 4.590-05 & $3.748-05$ & $3.168-05$ & $2.714-05$ \\
\hline 1 & 83 & $2.808-07$ & $3.844+05$ & $1.243-05$ & $1.240-05$ & $1.301-05$ & $1.349-05$ & $1.343-05$ & $1.375-05$ & $1.409-05$ \\
\hline 1 & 84 & & & $5.422-05$ & $4.938-05$ & $4.346-05$ & $3.760-05$ & $3.042-05$ & $2.564-05$ & $2.193-05$ \\
\hline 1 & 85 & $9.311-10$ & $1.278+03$ & $1.096-04$ & $1.003-04$ & 8.824-05 & 7.661-05 & $6.335-05$ & 5.404-05 & $4.673-05$ \\
\hline 1 & 86 & $4.085-05$ & $9.347+07$ & $8.206-05$ & $7.559-05$ & $6.746-05$ & 5.958-05 & $5.058-05$ & $4.431-05$ & 3.943-05 \\
\hline 1 & 87 & & & $1.717-05$ & $1.556-05$ & $1.366-05$ & $1.170-05$ & $9.011-06$ & 7.330-06 & $6.018-06$ \\
\hline 1 & 88 & & & 2.968-05 & $2.718-05$ & $2.395-05$ & $2.082-05$ & $1.730-05$ & $1.481-05$ & $1.285-05$ \\
\hline 1 & 89 & & & $2.352-05$ & $2.150-05$ & $1.926-05$ & $1.695-05$ & $1.370-05$ & $1.170-05$ & $1.016-05$ \\
\hline 1 & 90 & & & $4.268-05$ & 3.879-05 & $3.402-05$ & 2.924-05 & $2.275-05$ & $1.864-05$ & $1.543-05$ \\
\hline 1 & 91 & & & $3.273-05$ & $3.344-05$ & $3.588-05$ & $3.800-05$ & $3.920-05$ & $4.069-05$ & 4.199-05 \\
\hline 1 & 92 & & & $2.660-05$ & $2.408-05$ & $2.105-05$ & $1.800-05$ & $1.412-05$ & $1.159-05$ & $9.620-06$ \\
\hline 1 & 93 & & & $2.452-05$ & $2.461-05$ & $2.584-05$ & $2.687-05$ & $2.713-05$ & $2.788-05$ & 2.863-05 \\
\hline 1 & 94 & $6.190-07$ & $8.529+05$ & $6.393-04$ & $6.466-04$ & $6.666-04$ & $6.859-04$ & $7.067-04$ & $7.223-04$ & $7.352-04$ \\
\hline 1 & 95 & $6.415-15$ & $1.475-02$ & $1.281-05$ & $1.160-05$ & $1.012-05$ & 8.663-06 & $6.812-06$ & 5.599-06 & $4.656-06$ \\
\hline 1 & 96 & $2.137-06$ & $2.950+06$ & $3.739-05$ & 4.194-05 & 5.114-05 & $5.967-05$ & $6.702-05$ & 7.375-05 & 7.953-05 \\
\hline 1 & 97 & & & $1.047-04$ & $1.139-04$ & $1.310-04$ & $1.468-04$ & $1.610-04$ & $1.732-04$ & $1.834-04$ \\
\hline 1 & 98 & $1.339-02$ & $3.087+10$ & $9.501-04$ & $1.026-03$ & $1.185-03$ & $1.337-03$ & $1.481-03$ & $1.611-03$ & $1.729-03$ \\
\hline 1 & 99 & $1.535-11$ & $3.668+01$ & 5.233-04 & 4.803-04 & $4.247-04$ & 3.711-04 & $3.081-04$ & 2.643-04 & 2.299-04 \\
\hline 1 & 100 & & & $3.057-03$ & $3.102-03$ & $3.211-03$ & 3.316-03 & $3.431-03$ & $3.518-03$ & $3.590-03$ \\
\hline 1 & 101 & & & $1.523-04$ & $1.402-04$ & $1.240-04$ & $1.086-04$ & $9.180-05$ & 7.956-05 & 6.989-05 \\
\hline 1 & 102 & $\begin{array}{c}\text { 3.318-04 } \\
\text { available }\end{array}$ & $\begin{array}{c}8.000+08 \\
n \text { request }\end{array}$ & $4.659-04$ & $4.318-04$ & $3.880-04$ & $3.458-04$ & $2.993-04$ & 2.663-04 & $2.405-04$ \\
\hline 10 & 252 & & & $6.855-07$ & $6.430-07$ & $6.193-07$ & $5.730-07$ & $4.279-07$ & $3.571-07$ & 2.949-07 \\
\hline 10 & 253 & & & $1.269-06$ & $1.198-06$ & $1.184-06$ & $1.135-06$ & $9.514-07$ & 8.539-07 & 7.574-07 \\
\hline 10 & 254 & & & $1.389-06$ & $1.284-06$ & $1.157-06$ & $1.004-06$ & $6.968-07$ & $5.351-07$ & $4.070-07$ \\
\hline 10 & 255 & & & $1.013-06$ & $9.737-07$ & $1.012-06$ & $1.007-06$ & $8.562-07$ & $7.850-07$ & 7.056-07 \\
\hline 10 & 256 & & & $1.342-06$ & $1.199-06$ & $1.057-06$ & 9.009-07 & $6.337-07$ & 4.839-07 & $3.673-07$ \\
\hline 10 & 257 & & & $1.638-06$ & $1.503-06$ & $1.346-06$ & $1.163-06$ & 8.139-07 & $6.266-07$ & $4.784-07$ \\
\hline 10 & 258 & & & $2.377-06$ & $2.245-06$ & $2.210-06$ & $2.111-06$ & $1.769-06$ & $1.583-06$ & $1.398-06$ \\
\hline 10 & 259 & & & $1.092-05$ & $1.003-05$ & $9.011-06$ & 7.799-06 & 5.594-06 & $4.390-06$ & $3.437-06$ \\
\hline 10 & 260 & & & $1.327-05$ & $1.231-05$ & $1.135-05$ & $1.019-05$ & $7.876-06$ & $6.662-06$ & 5.709-06 \\
\hline 10 & 261 & & & $3.116-05$ & $3.215-05$ & $3.452-05$ & $3.648-05$ & $3.710-05$ & $3.821-05$ & $3.909-05$ \\
\hline 10 & 262 & & & $2.717-05$ & $2.470-05$ & $2.191-05$ & $1.880-05$ & $1.384-05$ & $1.097-05$ & $8.717-06$ \\
\hline 10 & 263 & & & $1.379-04$ & $1.379-04$ & $1.446-04$ & $1.499-04$ & $1.492-04$ & $1.526-04$ & $1.562-04$ \\
\hline 10 & 264 & & & $7.312-05$ & $6.630-05$ & 5.831-05 & 5.003-05 & $3.790-05$ & 3.053-05 & 2.479-05 \\
\hline 10 & 265 & & & $2.411-05$ & $2.229-05$ & $2.007-05$ & $1.752-05$ & $1.295-05$ & $1.040-05$ & $8.362-06$ \\
\hline 10 & 266 & & & $2.012-05$ & $1.926-05$ & $1.868-05$ & $1.789-05$ & $1.591-05$ & $1.499-05$ & $1.427-05$ \\
\hline 10 & 267 & & & $9.483-05$ & $8.652-05$ & 7.666-05 & $6.653-05$ & $5.146-05$ & $4.239-05$ & $3.534-05$ \\
\hline 10 & 268 & 5.984-14 & $7.455-02$ & $5.096-03$ & $5.377-03$ & 5.961-03 & $6.519-03$ & 7.063-03 & 7.534-03 & $7.950-03$ \\
\hline 10 & 269 & & & $2.758-05$ & $2.508-05$ & $2.213-05$ & $1.899-05$ & $1.419-05$ & $1.133-05$ & $9.106-06$ \\
\hline
\end{tabular}


Table XII-continued ...

\begin{tabular}{|c|c|c|c|c|c|c|c|c|c|c|}
\hline \multirow[t]{2}{*}{$\mathrm{i}$} & \multirow[t]{2}{*}{$\mathrm{j}$} & \multirow[t]{2}{*}{$g f$} & \multirow{2}{*}{$\begin{array}{c}A \\
\mathrm{~s}^{-1}\end{array}$} & \multicolumn{7}{|c|}{ Effective Collision strength $\Upsilon$} \\
\hline & & & & 0.5 & 1.0 & 2.0 & 3.0 & 4.0 & 5.0 & 6.0 \\
\hline 10 & 270 & $3.621-06$ & $4.512+06$ & $6.111-05$ & $6.356-05$ & $6.895-05$ & $7.356-05$ & $7.618-05$ & $7.916-05$ & $8.154-05$ \\
\hline 10 & 271 & & & $2.008-04$ & $1.824-04$ & $1.600-04$ & $1.375-04$ & $1.076-04$ & $8.848-05$ & $7.358-05$ \\
\hline 10 & 272 & & & $1.653-04$ & $1.503-04$ & $1.326-04$ & $1.143-04$ & $8.893-05$ & $7.306-05$ & $6.064-05$ \\
\hline 10 & 273 & & & $2.730-04$ & $2.793-04$ & $2.992-04$ & $3.169-04$ & $3.282-04$ & $3.418-04$ & $3.543-04$ \\
\hline 10 & 274 & $5.004-13$ & $1.040+00$ & $1.124-04$ & $1.116-04$ & $1.148-04$ & $1.170-04$ & $1.150-04$ & $1.160-04$ & $1.172-04$ \\
\hline 10 & 275 & 5.698-02 & $1.184+11$ & $1.421-04$ & $1.291-04$ & $1.134-04$ & $9.744-05$ & 7.694-05 & $6.363-05$ & $5.322-05$ \\
\hline 10 & 276 & $1.728-05$ & $2.154+07$ & $7.112-04$ & $7.729-04$ & 8.973-04 & $1.013-03$ & $1.118-03$ & $1.211-03$ & $1.291-03$ \\
\hline 10 & 277 & & & $8.041-06$ & $7.404-06$ & $6.551-06$ & $5.749-06$ & $4.925-06$ & $4.303-06$ & $3.812-06$ \\
\hline 10 & 278 & $2.326-03$ & $5.086+09$ & $2.424-05$ & $2.444-05$ & $2.587-05$ & $2.721-05$ & $2.812-05$ & 2.938-05 & $3.068-05$ \\
\hline 10 & 279 & $1.630-12$ & $3.573+00$ & $4.635-05$ & $4.257-05$ & $3.761-05$ & $3.281-05$ & $2.743-05$ & $2.360-05$ & $2.058-05$ \\
\hline 10 & 280 & $1.007-11$ & $2.209+01$ & $5.776-05$ & $5.321-05$ & 4.713-05 & 4.129-05 & $3.495-05$ & 3.034-05 & 2.669-05 \\
\hline 10 & 281 & $1.959-05$ & $2.579+07$ & $8.508-05$ & $8.086-05$ & $7.622-05$ & $7.168-05$ & $6.611-05$ & $6.251-05$ & $5.980-05$ \\
\hline 10 & 282 & & & $2.573-04$ & $2.585-04$ & 2.637-04 & $2.688-04$ & $2.745-04$ & 2.789-04 & 2.827-04 \\
\hline 10 & 283 & 9.424-11 & $1.242+02$ & $5.222-05$ & 4.821-05 & $4.279-05$ & $3.769-05$ & $3.255-05$ & 2.861-05 & 2.549-05 \\
\hline 10 & 284 & $2.347-11$ & $3.094+01$ & $6.948-05$ & 6.339-05 & $5.568-05$ & $4.814-05$ & $3.907-05$ & $3.292-05$ & $2.810-05$ \\
\hline 10 & 285 & & & $3.644-05$ & 4.171-05 & $5.297-05$ & $6.359-05$ & $7.292-05$ & 8.197-05 & $9.026-05$ \\
\hline 10 & 286 & $7.441-10$ & $9.813+02$ & 9.887-09 & $1.118-08$ & $1.129-08$ & $1.059-08$ & 6.731-09 & 5.166-09 & 3.815-09 \\
\hline 10 & 289 & & & $2.531-08$ & $2.464-08$ & $2.355-08$ & $2.127-08$ & $1.382-08$ & $1.047-08$ & 7.701-09 \\
\hline 10 & 290 & & & $1.223-04$ & $1.116-04$ & $9.806-05$ & $8.486-05$ & 6.918-05 & 5.845-05 & 5.004-05 \\
\hline 10 & 291 & & & $8.073-05$ & 7.493-05 & $6.852-05$ & $6.203-05$ & $5.341-05$ & $4.802-05$ & $4.391-05$ \\
\hline 10 & 292 & & & 6.098-04 & $6.368-04$ & 6.959-04 & 7.524-04 & 8.066-04 & 8.544-04 & 8.967-04 \\
\hline 10 & 293 & & & 4.974-07 & $4.448-07$ & 3.948-07 & $3.378-07$ & $2.363-07$ & $1.804-07$ & $1.367-07$ \\
\hline 10 & 294 & & & $5.228-07$ & $4.871-07$ & 4.526-07 & $4.087-07$ & $3.132-07$ & $2.650-07$ & 2.269-07 \\
\hline 10 & 295 & & & $9.128-07$ & $8.792-07$ & $8.501-07$ & $8.097-07$ & 7.018-07 & $6.575-07$ & $6.279-07$ \\
\hline 10 & 296 & & & $1.010-06$ & $9.251-07$ & $8.295-07$ & $7.164-07$ & 5.008-07 & $3.851-07$ & 2.939-07 \\
\hline 10 & 297 & & & $3.301-05$ & $3.000-05$ & $2.641-05$ & $2.274-05$ & $1.761-05$ & $1.441-05$ & $1.191-05$ \\
\hline 10 & 298 & & & $5.715-06$ & $5.266-06$ & $4.722-06$ & 4.111-06 & $3.023-06$ & $2.416-06$ & $1.933-06$ \\
\hline 10 & 299 & & & $6.783-06$ & 6.398-06 & $6.038-06$ & $5.603-06$ & $4.722-06$ & $4.271-06$ & 3.925-06 \\
\hline 10 & 300 & & & $1.419-05$ & $1.482-05$ & $1.617-05$ & $1.733-05$ & $1.794-05$ & $1.868-05$ & $1.929-05$ \\
\hline 10 & 301 & & & $1.284-05$ & $1.165-05$ & $1.029-05$ & 8.824-06 & $6.589-06$ & $5.265-06$ & $4.232-06$ \\
\hline 10 & 302 & & & $6.747-05$ & $6.134-05$ & $5.381-05$ & $4.626-05$ & $3.627-05$ & 2.986-05 & $2.487-05$ \\
\hline 10 & 303 & & & $3.783-05$ & $3.579-05$ & 3.397-05 & $3.196-05$ & 2.833-05 & 2.643-05 & $2.502-05$ \\
\hline 10 & 304 & $3.917-06$ & $5.170+06$ & $1.128-04$ & $1.213-04$ & $1.393-04$ & $1.560-04$ & $1.703-04$ & $1.836-04$ & $1.951-04$ \\
\hline 10 & 305 & $2.001-11$ & $4.409+01$ & $4.785-05$ & 4.396-05 & $3.876-05$ & 3.379-05 & $2.827-05$ & $2.432-05$ & $2.120-05$ \\
\hline 10 & 306 & & & $1.384-04$ & $1.271-04$ & $1.123-04$ & $9.797-05$ & $8.175-05$ & 7.024-05 & $6.119-05$ \\
\hline 10 & 307 & 7.329-06 & $9.695+06$ & 8.678-05 & 8.116-05 & 7.429-05 & $6.757-05$ & 5.979-05 & $5.445-05$ & 5.034-05 \\
\hline 10 & 308 & $1.210-12$ & $2.668+00$ & $9.489-05$ & $8.771-05$ & $7.784-05$ & $6.848-05$ & $5.847-05$ & 5.109-05 & 4.523-05 \\
\hline 10 & 309 & $8.768-05$ & $1.161+08$ & $1.183-04$ & $1.180-04$ & $1.206-04$ & $1.229-04$ & $1.240-04$ & $1.260-04$ & $1.280-04$ \\
\hline 10 & 310 & & & $1.069-03$ & $1.077-03$ & $1.103-03$ & $1.129-03$ & $1.158-03$ & $1.180-03$ & $1.198-03$ \\
\hline 10 & 311 & $1.705-09$ & $2.258+03$ & $1.606-04$ & $1.468-04$ & $1.290-04$ & $1.117-04$ & $9.115-05$ & 7.708-05 & $6.605-05$ \\
\hline 10 & 312 & & & 7.469-05 & $6.801-05$ & 5.961-05 & $5.135-05$ & $4.122-05$ & $3.443-05$ & 2.914-05 \\
\hline 10 & 313 & 2.059-09 & $2.730+03$ & $9.872-09$ & $1.060-08$ & $1.056-08$ & 9.833-09 & 6.156-09 & 4.649-09 & 3.364-09 \\
\hline 10 & 315 & & & $9.940-05$ & $9.065-05$ & 7.965-05 & $6.882-05$ & $5.545-05$ & 4.653-05 & 3.959-05 \\
\hline 10 & 317 & & & $2.289-08$ & $2.237-08$ & $2.135-08$ & $1.934-08$ & $1.247-08$ & $9.410-09$ & 6.876-09 \\
\hline 10 & 319 & & & $1.713-08$ & $1.969-08$ & $2.006-08$ & $1.888-08$ & $1.207-08$ & $9.342-09$ & 6.962-09 \\
\hline 10 & 320 & & & 3.059-08 & 2.969-08 & $2.828-08$ & $2.551-08$ & $1.670-08$ & $1.270-08$ & $9.402-09$ \\
\hline 10 & 322 & & & $1.531-04$ & $1.400-04$ & $1.232-04$ & $1.068-04$ & 8.749-05 & $7.421-05$ & $6.378-05$ \\
\hline 10 & 323 & & & $1.335-04$ & $1.238-04$ & $1.119-04$ & $1.004-04$ & $8.661-05$ & $7.730-05$ & 7.011-05 \\
\hline 10 & 324 & & & 5.193-05 & $4.752-05$ & $4.190-05$ & $3.644-05$ & $3.015-05$ & $2.576-05$ & $2.230-05$ \\
\hline 10 & 325 & & & $6.211-05$ & $6.133-05$ & $6.295-05$ & $6.401-05$ & $6.249-05$ & 6.299-05 & $6.377-05$ \\
\hline 10 & 326 & & & $4.254-07$ & $3.879-07$ & $3.507-07$ & $3.045-07$ & $2.078-07$ & $1.578-07$ & $1.181-07$ \\
\hline 10 & 327 & & & $4.795-07$ & $4.441-07$ & 4.094-07 & $3.650-07$ & $2.622-07$ & $2.112-07$ & $1.708-07$ \\
\hline 10 & 328 & & & $8.289-07$ & 7.921-07 & $7.616-07$ & $7.190-07$ & $6.090-07$ & 5.599-07 & $5.237-07$ \\
\hline 10 & 329 & & & $9.401-07$ & $8.629-07$ & $7.739-07$ & $6.702-07$ & $4.663-07$ & $3.580-07$ & $2.725-07$ \\
\hline 10 & 330 & & & 6.973-07 & $6.598-07$ & $6.203-07$ & 5.697-07 & $4.561-07$ & 3.999-07 & $3.561-07$ \\
\hline 10 & 331 & & & $9.551-07$ & $8.510-07$ & 7.494-07 & $6.359-07$ & 4.499-07 & $3.443-07$ & $2.622-07$ \\
\hline 10 & 332 & & & $1.871-03$ & $1.972-03$ & $2.185-03$ & $2.388-03$ & $2.585-03$ & $2.756-03$ & $2.907-03$ \\
\hline 10 & 333 & & & $1.163-06$ & $1.065-06$ & $9.503-07$ & $8.182-07$ & 5.759-07 & $4.440-07$ & $3.401-07$ \\
\hline 10 & 334 & & & $1.633-06$ & $1.559-06$ & $1.498-06$ & $1.414-06$ & $1.211-06$ & $1.119-06$ & $1.052-06$ \\
\hline 10 & 335 & & & 3.015-05 & $2.730-05$ & 2.403-05 & 2.059-05 & $1.562-05$ & $1.258-05$ & $1.023-05$ \\
\hline
\end{tabular}


Table XII-continued ...

\begin{tabular}{|c|c|c|c|c|c|c|c|c|c|c|}
\hline \multirow[t]{2}{*}{$\overline{\mathrm{i}}$} & \multirow[t]{2}{*}{$\overline{\mathrm{j}}$} & \multirow[t]{2}{*}{$\overline{g f f}$} & \multirow{2}{*}{$\begin{array}{c}A \\
\mathrm{~s}^{-1}\end{array}$} & \multicolumn{7}{|c|}{ 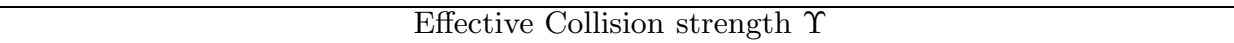 } \\
\hline & & & & 0.5 & 1.0 & 2.0 & 3.0 & 4.0 & 5.0 & 6.0 \\
\hline 10 & 336 & & & $3.928-05$ & $3.570-05$ & $3.159-05$ & $2.731-05$ & $2.106-05$ & $1.728-05$ & $1.434-05$ \\
\hline 10 & 337 & & & $5.070-06$ & $4.658-06$ & $4.169-06$ & $3.611-06$ & $2.596-06$ & $2.039-06$ & $1.599-06$ \\
\hline 10 & 338 & & & $6.105-06$ & $5.690-06$ & $5.212-06$ & $4.651-06$ & $3.579-06$ & $3.010-06$ & $2.565-06$ \\
\hline 10 & 339 & & & $1.328-05$ & $1.367-05$ & $1.465-05$ & $1.544-05$ & $1.564-05$ & $1.609-05$ & $1.647-05$ \\
\hline 10 & 340 & $6.092-09$ & $8.081+03$ & $1.247-05$ & $1.133-05$ & $1.001-05$ & $8.595-06$ & $6.364-06$ & $5.060-06$ & $4.040-06$ \\
\hline 10 & 341 & & & $7.908-05$ & $7.184-05$ & $6.295-05$ & 5.413-05 & $4.240-05$ & 3.489-05 & $2.903-05$ \\
\hline 10 & 342 & & & $6.886-05$ & $6.265-05$ & $5.518-05$ & $4.760-05$ & $3.714-05$ & $3.056-05$ & $2.541-05$ \\
\hline 10 & 343 & $7.645-14$ & $1.014-01$ & $1.013-04$ & $1.033-04$ & $1.103-04$ & $1.165-04$ & $1.202-04$ & $1.249-04$ & $1.293-04$ \\
\hline 10 & 344 & & & $9.280-06$ & $8.888-06$ & $8.593-06$ & $8.205-06$ & $7.327-06$ & $6.912-06$ & $6.603-06$ \\
\hline 10 & 345 & & & $1.137-05$ & $1.052-05$ & $9.446-06$ & $8.230-06$ & $6.108-06$ & $4.912-06$ & 3.961-06 \\
\hline 10 & 346 & $1.879-12$ & $4.155+00$ & $1.318-05$ & $1.194-05$ & $1.054-05$ & $9.035-06$ & $6.784-06$ & $5.436-06$ & $4.383-06$ \\
\hline 10 & 347 & & & $2.557-05$ & $2.645-05$ & $2.850-05$ & $3.023-05$ & $3.102-05$ & $3.212-05$ & $3.301-05$ \\
\hline 10 & 348 & & & $4.566-05$ & $4.502-05$ & $4.597-05$ & $4.653-05$ & $4.534-05$ & $4.549-05$ & $4.581-05$ \\
\hline 10 & 349 & $4.234-07$ & $5.620+05$ & $5.977-05$ & $5.433-05$ & $4.764-05$ & 4.099-05 & $3.243-05$ & $2.685-05$ & $2.250-05$ \\
\hline 10 & 350 & 4.429-02 & $9.798+10$ & $2.679-04$ & 2.901-04 & $3.356-04$ & $3.781-04$ & $4.160-04$ & $4.501-04$ & $4.794-04$ \\
\hline
\end{tabular}

